\title{
Completing the Is-a Structure in Description Logics Ontologies
}

by

\section{Zlatan Dragisic}

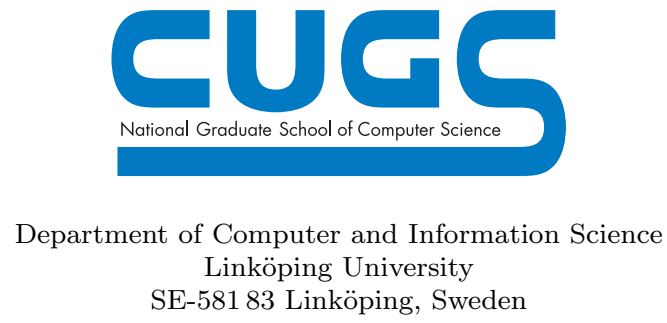

Linköping 2014 
This is a Swedish Licentiate's Thesis

Swedish postgraduate education leads to a doctor's degree and/or a licentiate's degree. A doctor's degree comprises 240 ECTS credits (4 year of full-time studies).

A licentiate's degree comprises 120 ECTS credits.

\section{Copyright (C) 2014 Zlatan Dragisic}

ISBN 978-91-7519-201-7

ISSN 0280-7971

Printed by LiU Tryck 2014

URL: http://urn.kb.se/resolve?urn=urn:nbn:se:liu:diva-110935 


\section{Abstract}

The World Wide Web contains large amounts of data and in most cases this data is without any explicit structure. The lack of structure makes it difficult for automated agents to understand and use such data. A step towards a more structured World Wide Web is the idea of the Semantic Web which aims at introducing semantics to data on the World Wide Web. One of the key technologies in this endeavour are ontologies which provide means for modeling a domain of interest.

Developing and maintaining ontologies is not an easy task and it is often the case that defects are introduced into ontologies. This can be a problem for semanticallyenabled applications such as ontology-based querying. Defects in ontologies directly influence the quality of the results of such applications as correct results can be missed and wrong results can be returned.

This thesis considers one type of defects in ontologies, namely the problem of completing the is-a structure in ontologies represented in description logics. We focus on two variants of description logics, the $\mathcal{E} \mathcal{L}$ family and $\mathcal{A L C}$, which are often used in practice.

The contributions of this thesis are as follows. First, we formalize the problem of completing the is-a structure as a generalized TBox abduction problem (GTAP) which is a new type of abduction problem in description logics. Next, we provide algorithms for solving GTAP in the $\mathcal{E L}$ family and $\mathcal{A L C}$ description logics. Finally, we describe two implemented systems based on the introduced algorithms. The systems were evaluated in two experiments which have shown the usefulness of our approach. For example, in one experiment using ontologies from the Ontology Alignment Evaluation Initiative 58 and 94 detected missing is-a relations were repaired by adding 54 and 101 is-a relations, respectively, introducing new knowledge to the ontologies.

This work has been supported by the Swedish National Graduate School of Computer Science (CUGS), the Swedish e-Science Research Center (SeRC) and Vetenskapsrådet (VR). 



\section{Acknowledgements}

At the beginning of my $\mathrm{PhD}$ studies this moment, even though half-way, seemed as a distant dream. The journey to this point was not easy and was full of ups and downs. However, the first half of the way is at its end and this thesis would not be possible without the help and support of a number of people along the way.

I would like to express my sincere gratitude to my supervisor Professor Patrick Lambrix for providing me with an opportunity to work on this project and all the guidance, help and advice given along the way. This made me improve and become better in what I am doing. Thank you for your patience and encouragement, especially in times when it seemed that things were not going my way.

I am extremely grateful to my secondary supervisors, Professor Nahid Shahmehri and Assistant Professor Fang Wei-Kleiner. Thank you for all the comments and discussions raised during our meetings which gave me a different perspective on my work and immensely improved it together with my critical thinking.

To all the former and current colleagues at ADIT I give my sincere thanks for making the work environment more enjoyable. I thank you for all lunches, discussions and various activities. They might not have always been productive but they raised some interesting questions about life, the universe and everything. I am also thankful to all the administrative staff, especially Anne, Eva, Inger, Karin and Marie, for their timely work and for making the administration hassle free.

To all my friends I am grateful for all the help and advice as well as all the fun moments we spent together during this time. Everything is so much easier with you around. I am also very thankful to Ekhiotz for proofreading this thesis and providing useful comments and suggestions.

My parents and my brother I thank for their words of encouragement and unequivocal support which made this "prolonged trip" to Sweden possible. Last but not least, I extend my deepest gratitude to my wife Svjetlana for being there at every step of the way, for patiently dealing with my concerns and being ready to listen about ontology debugging. Thank you for your love and your unconditional support!

Hvala Vam!

Zlatan Dragišić

October, 2014

Linköping, Sweden 



\section{Contents}

List of Figures $\quad$ ix

List of Tables

1 Introduction $\quad 1$

1.1 Motivation ................... 1

1.2 Problem formulation . . . . . . . . . . . . . . . 4

1.3 Contributions . . . . . . . . . . . . . . . 5

1.4 List of publications . . . . . . . . . . . . . . . . . . . . . . . . . . . 6

1.5 Thesis outline . . . . . . . . . . . . . 7

2 Preliminaries 9

2.1 Ontologies ........................ 9

2.1.1 Classifications . . . . . . . . . . . . . 11

2.2 Description Logics . . . . . . . . . . . . . . . . . . . . . . . . . . . . . . . . . . . . 12

$2.2 .1 \quad \mathcal{E} \mathcal{L}$ family . . . . . . . . . . . . . . . . . . . . . . 14

$2.2 .2 \quad \mathcal{A L C} \ldots \ldots \ldots \ldots \ldots$

2.3 Reasoning in description logics . . . . . . . . . . . . . 16

2.3.1 Tableaux reasoning . . . . . . . . . . . . . 17

2.4 Debugging ontologies . . . . . . . . . . . . . . . . 19

2.4.1 Classification of defects . . . . . . . . . . . 20

2.5 Abduction in description logics . . . . . . . . . . . . . 21

2.5.1 Constraints on solutions . . . . . . . . . . 21

3 Repairing incomplete ontologies - framework 23

3.1 Abduction Framework . . . . . . . . . . . . . . . . . . . . . . 24

3.2 Solutions with preference criteria . . . . . . . . . . . 27

3.3 Debugging in practice ............... . . 31

3.3.1 General observations . . . . . . . . . . . . . 31

3.3.2 Lessons for an existing system . . . . . . . . . . 31

4 Repairing missing is-a structure in $\mathcal{E} \mathcal{L}$ ontologies 33

4.1 Algorithm $-\mathcal{E} \mathcal{L} \ldots \ldots . \ldots . \ldots 34$

4.2 Algorithm $-\mathcal{E} \mathcal{L}++\ldots \ldots . \ldots \ldots 37$ 
4.3 System . . . . . . . . . . . . . . . . . . . 41

4.4 Experiments . . . . . . . . . . . . . . . . . . 43

4.4.1 Experiment 1 - OAEI Anatomy . . . . . . . . . 43

4.4.2 Experiment 2 - BioTop . . . . . . . . . . . 46

4.4 .3 Lessons Learned . . . . . . . . . . . . . . . 47

5 Repairing missing is-a structure in $\mathcal{A L C}$ ontologies $\quad 49$

5.1 Algorithm . . . . . . . . . . . . . . . 50

5.2 System . . . . . . . . . . . . . . . . . 55

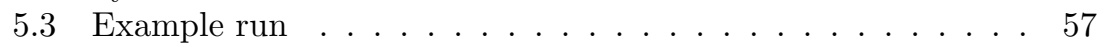

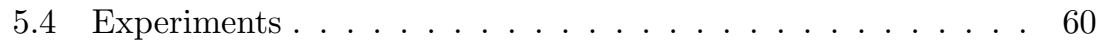

5.4 Lessons learned . . . . . . . . . . . . . . . 61

6 Related work $\quad 65$

6.1 Completing ontologies ... . . . . . . . . . . . 65

6.2 Detecting missing is-a relations . . . . . . . . . . . . . 66

6.3 Debugging semantic defects . . . . . . . . . . . . . . 69

6.4 Abductive reasoning in description logics . . . . . . . . . . 71

$\begin{array}{llr}7 & \text { Conclusion and Future Work } & \mathbf{7 5}\end{array}$

7.1 Future work . . . . . . . . . . . . 77

$\begin{array}{ll}\text { Bibliography } & 81\end{array}$ 


\section{List of Figures}

1.1 A part of Adult Mouse Anatomy - AMA ontology concerning the concept joint. . . . . . . . . . . . . . . . . . . 3

2.1 A knowledge base - example. . . . . . . . . . . . . . 13

2.2 Transformation rules (e.g. [16]). . . . . . . . . . . . . . 17

2.3 Completion graph for Professor $\sqcap \neg$ Teacher. . . . . . . . . 18

3.1 Small example based on the ontology from Figure 1.1. . . . . 26

$4.1 \quad$ Small $\mathcal{E} \mathcal{L}$ example. . . . . . . . . . . . . . . . . . . . 37

4.2 Small $\mathcal{E L}^{++}$example. . . . . . . . . . . . . . . . . . 38

4.3 System for repairing $\mathcal{E} \mathcal{L}$ ontologies - screenshots. . . . . . . 42

$5.1 \quad$ Small $\mathcal{A L C}$ example. . . . . . . . . . . . . . . . . . . . . 53

5.2 Tbox $\mathrm{T}$ from Figure 5.1 represented as an acyclic $\mathcal{A L C}$ terminology. . . . . . . . . . . . . . . . . 54

5.3 Screenshot - Validating is-a relations in a repairing action. . . 55

5.4 Screenshot - Repairing using Source and Target sets. . . . . . 56

5.5 Completion graph for MyPizza $\sqcap \neg$ FishyMeatyPizza. . . . 63

5.6 Creating $R_{\mathcal{A}}$ for the leaf ABoxes related to MyPizza $\sqsubseteq$ FishyMeatyPizza. . . . . . . . . . . . . . . . . . . 64 



\section{List of Tables}

2.1 The $\mathcal{E} \mathcal{L}$ family - Syntax and Semantics. . . . . . . . . . . 14

$2.2 \mathcal{A L C}$ - Syntax and Semantics. . . . . . . . . . . . 15

3.1 Different combinations of cases for $T$, Or and $M \ldots \ldots 27$

4.1 Results for debugging AMA - Adult Mouse Anatomy ontology. 44

4.2 Source and Target set sizes for debugging AMA - Adult Mouse Anatomy ontology. The $\mathrm{x} / \mathrm{y} / \mathrm{z}$ values represent the sizes for iteration 1,2 and 3 , respectively. . . . . . . . . . . 44

4.3 Results of debugging NCI-A - Human Anatomy ontology . . . 45

4.4 Source and Target set sizes for debugging NCI-A - Human Anatomy ontology. The $\mathrm{x} / \mathrm{y} / \mathrm{z}$ values represent the sizes for iteration 1,2 and 3 , respectively. . . . . . . . . . . 45

4.5 Results for debugging the BioTop ontology. . . . . . . . . . 46

4.6 Source and Target set sizes for debugging the BioTop ontology. The $\mathrm{x} / \mathrm{y} / \mathrm{z} / \mathrm{u}$ values represent the sizes for iteration 1,2 , 3 and 4 , respectively. . . . . . . . . . . . 46 



\section{Chapter 1}

\section{Introduction}

\subsection{Motivation}

The World Wide Web (WWW) is a network of web sites interconnected via hyperlinks. It is growing rapidly and as of October 2014 it is estimated to contain around 1 billion web sites $[2,4]$. Data on the WWW is available in different formats, such as documents, databases, images and videos. This data has often only limited structure. For example, web pages are often only semi-structured containing only machine-readable meta-data needed for a correct presentation of a web site in a browser. The actual content (body) of web-pages is human-readable and often without any explicit structure.

The lack of structure makes the automation of more sophisticated queries which require the understanding of the meaning of the data a problem. As a result of this, large amounts of useful data on the WWW are not being used to their full potential. For example, querying for the age of a person in a document containing the birth year of that person would already pose a difficulty for an automated agent. The agent would not have an understanding of the concept age and how it relates to the birth year. In order to achieve queries like this, a preprocessing step such as knowledge extraction is often required. However, these preprocessing steps are in many cases incomplete and inaccurate and require human intervention to validate the extracted knowledge.

In some cases it may be necessary to combine information from multiple sources to answer a specific query. For example, in order to answer a query such as "Which actor from the movie Inception has the most Academy Award nominations?" we might have to access information on two separate web pages, one containing the cast of Inception and one with the list of all Academy Award nominees. To answer such queries manually it is necessary to navigate to multiple data sources and assemble the information. These data sources can be heterogeneous, having different data models or data in different formats which would limit an automated agent's ability to answer 
such queries. The reason for this is again the lack of structure on the current WWW which limits the ability of the agent to relate concepts in different sources.

As a way of dealing with these issues Berners-Lee et al. [18] proposed the idea of a Semantic Web. The Semantic Web is supposed to be an extension of the WWW which would structure meaningful information on the Web, thus making it possible for automated agents to execute more sophisticated tasks. In order to do this, current human readable content on the WWW has to be annotated with semantic labels which would be used by automated agents to extract meaning. Technologies used to achieve this are Extensible Markup Language (XML) and Resource Description Framework (RDF) which provide a syntax needed for defining semantic labels as well as a framework for defining statements about resources on the WWW. However, same as the WWW, the Semantic Web is decentralized and there are no naming standards when it comes to semantic labels. This means that two sources might use different labels for the same concept which causes a problem when integrating information from multiple data sources. One way to deal with this kind of ambiguity is to model the domain of interest, i.e. describe which type of objects (i.e. concepts) exist, which kind of properties they possess and how they relate to each other. On the Semantic Web this is done using ontologies which provide means for defining a formal vocabulary of a domain of interest. On top of this, ontologies also allow for inference and reasoning which makes it possible to infer implicit knowledge from ontologies. Ontologies enable automated agents to acquire an understanding of the underlying data as well as provide a vocabulary for communication with other agents.

While ontologies are useful, developing ontologies is not an easy task, and often the resulting ontologies are incorrect or incomplete which might lead to wrong conclusions being derived or valid conclusions being missed. Defects in ontologies can take different forms and range from those which are easy to detect and resolve such as syntactic defects, representing errors in syntax in the ontology representation, to more severe ones such as semantic and modeling defects. Semantic defects represent problems within the logic in the ontology, while examples of modeling defects are missing or wrong relations. Domain knowledge is required to detect and resolve modeling defects. In this work, we focus on incomplete ontologies, more specifically ontologies with missing relations. In addition to being problematic for the correct modeling of a domain, incomplete ontologies also influence the quality of semantically-enabled applications.

Incomplete ontologies when used in semantically-enabled applications can lead to valid conclusions being missed. In ontology-based search, queries are refined and expanded by moving up and down the hierarchy of concepts. Incomplete structure in ontologies influences the quality of the search results. As an example, suppose we want to find articles in PubMed [5] using the MeSH [3] term Scleral Diseases. PubMed is a database of references and 


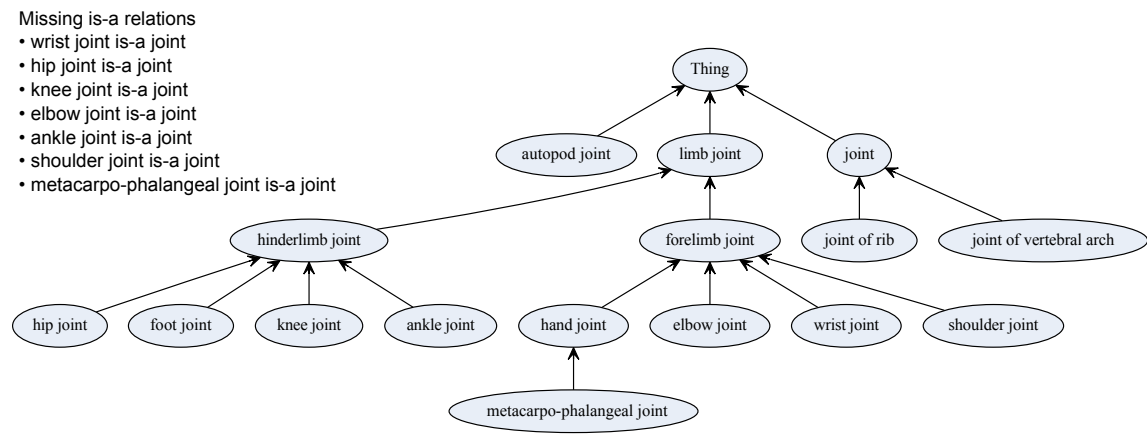

Figure 1.1: A part of Adult Mouse Anatomy - AMA ontology concerning the concept joint.

abstracts primarily from the life sciences literature and $\mathrm{MeSH}$ is a thesaurus used for indexing PubMed records. By default the query will follow the hierarchy of MeSH and include more specific terms for searching, such as Scleritis. If the relation between Scleral Diseases and Scleritis is missing in MeSH, we will miss 948 articles in the search result, which is about $57 \%$ of the original result ${ }^{1}$.

Completing ontologies consists of two phases, detection and repair. In the detection phase missing relations are detected and in the repairing phase the detected missing relations are made derivable in the ontology. There are different ways to detect missing relations. One way is inspection by domain experts. Another way is using linguistic patterns, e.g. if we have concepts $\mathrm{X}$ and $\mathrm{Y}$ in the ontology and a statement " $\mathrm{X}$ such as $\mathrm{Y}$ " in some text, then a relation $\mathrm{Y}$ is-a $\mathrm{X}$ is a possible missing relation in the ontology. Although there are many approaches to detect missing relations, these approaches, in general, do not detect all missing relations. For instance, although the precision for the linguistic patterns approaches is high, their recall is usually very low.

In this thesis we deal with missing is-a relations ( $\sqsubseteq)$ which are relations between concepts which define that some concept is a type of some other concept e.g. Tree $\sqsubseteq$ Plant. We assume that the detection phase has been performed. Further, we assume that we have obtained a set of missing is-a relations for a given ontology and focus on the repairing phase. In the ideal case where our set of missing is-a relations contains all missing is-a relations, the repairing phase is easy. We just add all missing is-a relations to the ontology and a reasoner can compute all logical consequences. However, when the set of missing is-a relations does not contain all missing is-a relations and this is the common case - there are different ways to repair the ontology.

For instance, Figure 1.1 shows a small ontology representing a part of the

\footnotetext{
${ }^{1}$ PubMed accessed on 14-10-2014
} 
Adult Mouse Anatomy (AMA) ontology concerning joint, that is relevant for our discussions. A list of detected missing is-a relations is given on the left side. Adding these relations to the ontology will repair the missing is-a structure. However, there are other more interesting possibilities. The missing is-a structure can be repaired by adding limb-joint $\sqsubseteq$ joint. Further, this is-a relation is correct according to the domain and constitutes a new is-a relation that was not derivable from the ontology and not originally detected by the detection algorithm. To illustrate why limb-joint $\sqsubseteq$ joint repairs the missing is-a structure consider the missing is-a relation wristjoint $\sqsubseteq$ limb-joint. As relation wrist-joint $\sqsubseteq$ limb-joint is already derivable from the ontology then adding limb-joint $\sqsubseteq$ joint would make wrist-joint $\sqsubseteq$ joint derivable in the ontology. Similar reasoning holds for the other missing is-a relations in the set. We also note that from a logical point of view, adding limb-joint $\sqsubseteq$ joint-of-rib also repairs the missing is-a structure. However, from the point of view of the domain, this solution is not correct. Therefore, as is the case for all approaches for dealing with modeling defects, a domain expert needs to validate the logical solutions.

\subsection{Problem formulation}

As the previous discussion pointed out, incomplete structure in ontologies can lead to incomplete results in semantically-enabled applications. To deal with this problem it is necessary to detect and resolve missing relations in the ontology. So far most work on completing missing structure in ontologies has focused on taxonomies, from a knowledge representation point of view, a simple type of ontologies containing only concepts and is-a relations (e.g. [70], [68]). However, in recent years there has been an increasing use of ontologies represented in more expressive knowledge representation languages. Examples of this can be found in the biomedical domain, where ontology repositories such as BioPortal [1] contain a large number of ontologies ranging from relatively simple ontologies to very expressive ontologies [52]. Another example of an expressive ontology used in practice is SNOMED Clinical Terms (SNOMED CT) [6] ontology which is the largest collection of medical terms in the world with more than 300,000 concepts with formal logic-based definitions.

The goal of our work is to develop a framework for repairing missing the is-a structure in more expressive lightweight ontologies. These more expressive ontologies are usually logic-based meaning that they are defined using some formal logic. In the case of logic-based ontologies, description logics are often used for the formalization. There are different varieties of description logics, and in our work we focus on two of them, the $\mathcal{E} \mathcal{L}$ family and $\mathcal{A L C}$ which are used for representation of a number of ontologies in practice. Many of these ontologies are used in the life sciences which are one of the first as well the biggest adopters of the Semantic Web technologies [91]. 
The thesis addresses the following research question:

How to repair missing is-a structure in lightweight ontologies?

To answer the research question we pursue three specific objectives:

- To formalize the problem of repairing missing is-a structure in lightweight ontologies;

- To develop algorithms for repairing missing is-a structure in lightweight ontologies;

- To develop a system for repairing missing is-a structure in lightweight ontologies and analyse the usefulness of such system;

\subsection{Contributions}

The contributions of this thesis are as follows:

With respect to the objective To formalize the problem of repairing missing is-a structure in lightweight ontologies:

- We have formalized the problem of completing the is-a structure in ontologies as a generalized TBox abduction problem (GTAP) which is an extension of a TBox abduction problem [38]. Further, we introduced different preference criteria relevant for completing the is-a structure. These criteria also take into account knowledge added to an ontology. This is in contrast with preference criteria in logic-based abduction which usually emphasise the solution size.

With respect to the objective To develop algorithms for repairing missing is-a structure in lightweight ontologies:

- We have developed algorithms for completing the is-a structure in more expressive ontologies. In this thesis we considered logic-based ontologies in the $\mathcal{E} \mathcal{L}$ family and $\mathcal{A L C}$ for which we developed two algorithms, an $\mathcal{E} \mathcal{L}$ family algorithm which utilizes different patterns to identify solutions to GTAP and an $\mathcal{A L C}$ algorithm which is more general and is based on a tableaux reasoning algorithm.

With respect to the objective To develop a system for repairing missing is-a structure in lightweight ontologies and analyse the usefulness of such system:

- We have developed systems for repairing missing is-a structure in ontologies based on the $\mathcal{E} \mathcal{L}$ family and $\mathcal{A L C}$.

- We have performed example runs and experiments on the developed systems. The developed systems have been tested on a number of ontologies with different level of expressivity. In the first experiment using the anatomy ontologies from the Ontology Alignment Evaluation 
Initiative the detected 94 and 58 missing is-a relations were repaired by adding 101 and 54 is-a relations, respectively. Out of these, 47 in the first experiment and 10 in the second represent new knowledge which was not identified by the detection algorithm. In the second experiment using the BioTop ontology, 47 missing is-a relations were repaired with 41 is-a relations out of which 40 represent new is-a relations. Given this, our approach for completing the is-a structure can also be seen as a detection method that takes already found missing is-a relations as input.

\subsection{List of publications}

This thesis is based on the following publications:

\section{Conference articles}

- P. Lambrix, Z. Dragisic, and V. Ivanova. Get my pizza right: Repairing missing is-a relations in $\mathcal{A L C}$ ontologies, In Proceedings of the 2nd Joint International Semantic Technology Conference - JIST 2012, volume 7774 of Lecture Notes in Computer Science, pages 17-32, Nara, Japan, 2012.

- Z. Dragisic, P. Lambrix, and F. Wei-Kleiner. Completing the isa structure of biomedical ontologies, In Proceedings of the 10th International Conference on Data Integration in the Life Sciences DILS 2014, volume 8574 of Lecture Notes in Bioinformatics, pages 66-80, Lisbon, Portugal, 2014.

- F. Wei-Kleiner, Z. Dragisic, and P. Lambrix. Abduction Framework for Repairing Incomplete $\mathcal{E} \mathcal{L}$ Ontologies: Complexity Results and Algorithms, In Proceedings of the 28th AAAI Conference on Artificial Intelligence - AAAI 2014, pages 1120-1127, Quebec City, Canada, 2014.

\section{Workshop articles}

- P. Lambrix, F. Wei-Kleiner, Z. Dragisic, and V. Ivanova. Repairing Missing Is-a structure in ontologies is an abductive reasoning problem, In Proceedings of the 2nd International Workshop on Debugging Ontologies and Ontology Mappings - WoDOOM 2013, volume 999 of CEUR Workshop Proceedings, pages 33-44, Montpellier, France, 2013.

- Z. Dragisic, P. Lambrix, and F. Wei-Kleiner. A System for Debugging Missing Is-a Structure in $\mathcal{E} \mathcal{L}$ Ontologies, In Proceedings of the 3rd International Workshop on Debugging Ontologies and 
Ontology Mappings - WoDOOM 2014, volume 1162 of CEUR Workshop Proceedings, pages 51-58, Anissaras/Hersonissou, Greece, 2014. Demo.

The following publications are related to the content of the thesis :

\section{Book chapter}

- P. Lambrix, V. Ivanova, and Z. Dragisic. Contributions of LiU/ADIT to Debugging Ontologies and Ontology Mappings, In Lambrix, (ed), Advances in Secure and Networked Information Systems - The ADIT Perspective, pages 109-120, LiU Tryck / LiU Electronic Press, 2012.

\section{Workshop article}

- B. C. Grau, Z. Dragisic, K. Eckert, J. Euzenat, A. Ferrara, R. Granada, V. Ivanova, E. Jimenez-Ruiz, A. O. Kempf, P. Lambrix, A. Nikolov, H. Paulheim, D. Ritze, F. Scharffe, P. Shvaiko, C. Trojahn and O. Zamazal. Results of the Ontology Alignment Evaluation Initiative 2013, In Proceedings of the 8th International Workshop on Ontology Matching - OM 2013, volume 1111 of CEUR Workshop Proceedings, pages 61-100, Sydney, Australia, 2013.

\subsection{Thesis outline}

The rest of the thesis is organized as follows:

Chapter 2 provides background on ontologies and description logics. In addition, the chapter extends the discussion about ontology debugging as well as gives intuitions of abductive reasoning in logic-based ontologies.

Chapter 3 formalizes the abduction framework for debugging the is-a structure of ontologies, i.e. defines the problem as well as a number of preference criteria on solutions. The chapter also analyses how different properties of the ontology, the set of is-a relations to repair and domain expert influence the existence of solutions. Finally, the consequences of this analysis for debugging in practice are explored.

Chapter 4 introduces an algorithm for debugging missing is-a structure in the $\mathcal{E} \mathcal{L}$ family of ontologies based on our formalization. A working system based on the algorithm is described and evaluated in two experiments.

Chapter 5 describes an algorithm for debugging missing is-a structure in $\mathcal{A L C}$ ontologies based on our formalization. The chapter also presents 
a system based on the algorithm together with an example run and experiments discussion.

Chapter 6 covers an overview of related work with focus on debugging missing is-a structure.

Chapter 7 provides a discussion of presented solutions as well as directions for future work. 


\section{Chapter 2}

\section{Preliminaries}

This chapter presents the background of areas relevant for this thesis. The chapter is organized as follows. First, in Section 2.1 we present the concept of ontologies and discuss components, uses and a classification of ontologies. In Section 2.2 we provide the details about description logics and present variants of description logics relevant for this work. Reasoning in description logics is discussed in Section 2.3. In addition, Section 2.3 also discusses tableaux reasoning which is an approach for reasoning in description logics used in this thesis. Details about ontology debugging and an overview of different defects in ontologies is given in Section 2.4. Finally, Section 2.5 gives an overview of different abduction problems in description logics and discusses different preference criteria on solutions to abductive queries.

\section{$2.1 \quad$ Ontologies}

The term ontology comes from philosophy where it is a study of existence and the nature of being. It tries to answer questions such as "What does it mean to exist?" or "What can be said to exist?". In computer science the term was first used by McCarthy [78] in 1980 when discussing a new form of logic where he suggested that ontologies can be used as a way of expressing common sense knowledge. However, the ontologies were still discussed in philosophical terms until the mid 80s when Alexander et al. [10] proposed a language for encoding ontological knowledge about the domain. This is recognized as the first use of the term ontology from a computer science perspective and a step away from philosophy [97]. Since then ontologies were adopted in many Computer Science communities, specifically in Artificial Intelligence where ontology engineering became one of the important knowledge representation formalisms.

There are a number of definitions of ontologies in Computer Science. One of the first ones is by Neches et al. [80] which states: "An ontology defines the basic terms and relations comprising the vocabulary of a topic area as 
well as the rules for combining terms and relations to define extensions to the vocabulary". Probably the most cited one in literature is by Gruber [43] where an ontology is defined as "an explicit specification of a conceptualization". Studer et al. [93] extended this definition and defined an ontology as "a formal, explicit specification of a shared conceptualization".

These definitions are related by the idea of conceptualization, i.e. an abstraction or a simplified view of the domain in question. The specification of this conceptualization should be explicit, i.e., that the types of concepts, their relations and their use are explicitly defined and formal, meaning that they are machine readable [93]. Studer et al. [93] also emphasized the need for this conceptualization to be "shared" meaning that it is a result of a consensus and does not only encode knowledge of a single individual.

Ontologies differ in what kind of knowledge they can represent, i.e. which knowledge representation formalisms they are based on. Given this, different ontology components can be identified (e.g. [92, 66, 39]). Corcho et al. [26] define a minimal set of components that different kinds of ontologies share:

- concepts (classes) - represent types of objects in the domain. Objects can be both abstract and concrete, as well as simple or complex, e.g. Man, Endocarditis, Carditis, PathologicalPhenomenon.

- instances (individuals) - represent instantiations of concepts, i.e. actual objects for example John. The assertion Man(John) represents that John is an instance of concept Man.

- relations (properties, roles) - represent relations between concepts in the domain. Stevens et al. [92] define two types of relations:

- taxonomical - which represent relations which organize concepts into hierarchies. The two most used types of these are specialization relations (is-a, subconcept, subclass) and partitative relations (part-of). For example, Endocarditis is-a Carditis represents a specialization relation which defines that Endocarditis is a type of Carditis. An example of a partitative relation is the relation Lower jaw part-of Jaw.

- associative - which relate concepts across concept hierarchies (e.g. is-caused-by, has-associated-process, etc.).

- axioms - model statements which are always true in a domain which can not be defined by other components [26]. Axioms are used to define such statements as cardinality restrictions (Man has exactly one Jaw), disjoint concepts (Endocarditis is not a Fracture) as well as general statements about the domain (e.g. Endocarditis is-a InflammatoryProcess and has-location Endocardium). This kind of statements are useful for verifying if the knowledge in the ontology is consistent as well for inferring new knowledge not explicitly defined in the ontology $[26]$. 
Ontologies have a number of uses, such as the following [65]:

- they are used as a means for communication between people and organizations;

- enable knowledge reuse and sharing;

- they provide a basis for interoperability between systems;

- ontologies are used for data integration;

- they are used as a repository of information;

In addition to being a key technology for the Semantic Web, ontologies are used in a variety of areas:

- Software Engineering - ontologies can be used in all phases of software engineering life-cycle, e.g. as means for representing different artefacts of a development process [48]. Ontologies are also used to support the systematic review process in Software Engineering [28];

- Artificial Intelligence - ontologies provide means for representing common sense knowledge [74];

- Computer Security - ontologies are used to encode properties of resources and different threats [63, 51];

- Biomedicine - ontologies are often used as knowledge repositories and means for data integration across heterogeneous data sources [81];

\subsubsection{Classifications}

Depending on the expressiveness of the knowledge representation formalism used for defining ontologies a number of categories of ontologies can be defined. One of the first such classifications was introduced by Lassila and McGuinness [73] (later extended by [94]). This work defined an ontology spectrum which spans from inexpressive lightweight ontologies represented in informal languages towards very expressive ontologies represented in formal languages.

- Glossaries and Data Dictionaries - represent the simplest types of ontologies, essentially a list of terms. An example of this kind of ontologies are controlled vocabularies. In the case of glossaries terms are associated with a meaning specified in natural language.

- Thesauri and taxonomies - represent ontologies which are a list of terms with a fixed set of relations between them. For example thesauri can define relations such as hyponym, antonym, synonym (e.g. WordNet [9]). In the case of taxonomies, terms are organized into an is-a hierarchy. 
- Ontologies represented using metadata, XML, schemas and data models - ontologies in this category can define concept hierarchies, attributes, relations and axioms.

- Ontologies represented using logical languages - represents the most expressive kind of ontologies based on a formal language (logic). The formal languages provide syntax and well-defined semantics as well as reasoning mechanisms such as consistency checking. Description logics is an example of a formal language widely used for defining ontologies.

A similar classification is given in [65] where ontologies are classified based on the components and the information they contain.

\subsection{Description Logics}

Description logics is a family of knowledge representation formalisms used for representing knowledge in an application domain. In description logics an application domain is defined in terms of concepts which are used to describe entities in the domain. One of the main reasons for the popularity of description logics in knowledge representation systems is the emphasis on the reasoning possibilities which allow for inferring implicit knowledge from explicitly defined descriptions.

There are three main building blocks in description logic languages [15]:

- atomic concepts - unary predicates, representing types or sets of objects in the domain, e.g. Professor, Course, ResearchProject

- atomic roles - binary predicates, representing binary relations between the objects in the domain, e.g. teaches, worksOn

- individuals - constants, representing actual objects in the domain, e.g. john, mary, semanticweb101

A vocabulary of a description logic language can be defined as a triplet $\left(N_{C}, N_{R}, N_{I}\right)$ where $N_{C}$ is a set of atomic concepts, $N_{R}$ is a set of atomic roles and $N_{I}$ is a set of individual names. Complex concept and role descriptions in the application domain are formed by combining the basic building blocks and logical constructors such as conjunction $(\sqcap)$, disjunction $(\sqcup)$, existential quantifier $(\exists)$, etc.

The semantics of concept descriptions is defined in terms of interpretations. An interpretation $\mathcal{I}$ consists of a non-empty set $\Delta^{\mathcal{I}}$ and an interpretation function. ${ }^{\mathcal{I}}$ which assigns to each atomic concept $A \in N_{C}$ a subset $A^{\mathcal{I}} \subseteq \Delta^{\mathcal{I}}$, to each atomic role $r \in N_{R}$ a relation $r^{\mathcal{I}} \subseteq \Delta^{\mathcal{I}} \times \Delta^{\mathcal{I}}$, and to each individual name $a \in N_{I}$ an object $a^{\mathcal{I}} \in \Delta^{\mathcal{I}}$.

A knowledge base in description logics is an ordered pair $(\mathcal{T}, \mathcal{A})$ consisting of a terminological component called TBox $(\mathcal{T})$ and an assertional component called $\operatorname{ABox}(\mathcal{A})$. 


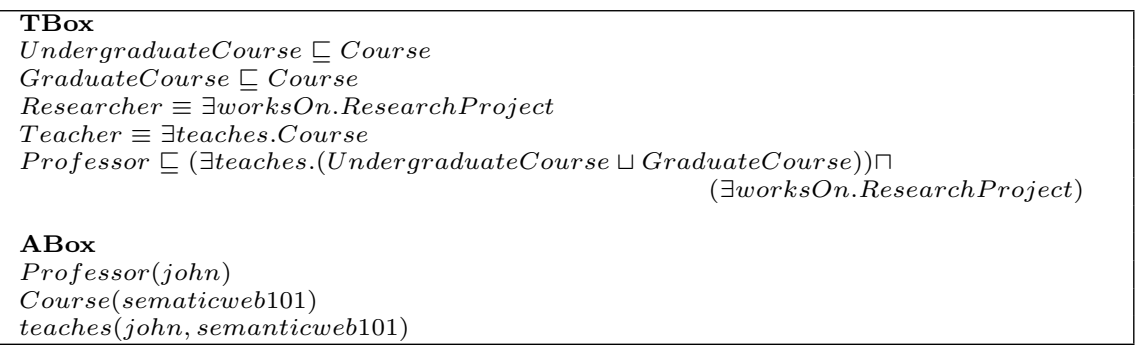

Figure 2.1: A knowledge base - example.

A TBox contains a finite set of terminological axioms i.e. statements about how concepts and roles relate to each other. These axioms, in the general case, are of the form:

$$
\begin{aligned}
& C \sqsubseteq D(r \sqsubseteq s) \\
& C \equiv D(r \equiv s)
\end{aligned}
$$

where $C$ and $D$ are concepts (atomic or complex) and $r$ and $s$ are roles (atomic or complex) [15]. The first type of axioms are called subsumption axioms (also known as inclusions, specializations, is-a relations). With regards to the semantics, an interpretation $\mathcal{I}$ satisfies a subsumption axiom $C \sqsubseteq D(r \sqsubseteq s)$ if it holds that $C^{\mathcal{I}} \subseteq D^{\mathcal{I}}\left(r^{\mathcal{I}} \subseteq r^{\mathcal{I}}\right)$. If an interpretation $\mathcal{I}$ satisfies an axiom (or set of axioms) then $\mathcal{I}$ is a model of this axiom (or a set of axioms). Axioms concerning concepts are also known as general concept inclusions (GCI) while axioms concerning roles are known as general role inclusions (GRI). The second type of axioms are equivalence axioms. An interpretation $\mathcal{I}$ satisfies an equivalence $C \equiv D(r \equiv s)$ if it holds that $C^{\mathcal{I}}=D^{\mathcal{I}}\left(r^{\mathcal{I}}=s^{\mathcal{I}}\right)$. Equivalence $C \equiv D(r \equiv s)$ can also be represented with two subsumption axioms: $C \sqsubseteq D$ and $D \sqsubseteq C(r \sqsubseteq s$ and $s \sqsubseteq r)$. If the left hand side of an equivalence axiom is an atomic concept then these axioms are also known as concept definitions.

An ABox contains assertional knowledge, i.e. statements about the membership of individuals to concepts (concept assertions) and relations between individuals (role assertions). For example, Professor(john), Course(semanticweb101) are concept assertions and teaches(john, semanticweb101) is a role assertion where john and semanticweb101 are individuals, Professor and Course are atomic concepts and teaches is an atomic role. An interpretation $\mathcal{I}$ is a model of an ABox if for every concept assertion $C(a)$ it holds that $a^{\mathcal{I}} \in C^{\mathcal{I}}$ and for every role assertion $r(a, b)$ it holds that $\left(a^{\mathcal{I}}, b^{\mathcal{I}}\right) \in r^{\mathcal{I}}$. An interpretation is a model for a knowledge base if it is a model for the TBox and the ABox.

An example description logic knowledge base is given in Figure 2.1. In this example, Course, UndergraduateCourse, GraduateCourse, Teacher, ResearchProject, Researcher and Professor are atomic concepts, teaches 


\begin{tabular}{|c|c|c|}
\hline Name & Syntax & Semantics \\
\hline \hline top & $\top$ & $\Delta^{\mathcal{I}}$ \\
\hline bottom & $\perp$ & $\emptyset$ \\
\hline nominal & $\{a\}$ & $\left\{a^{\mathcal{I}}\right\}$ \\
\hline conjunction & $C \sqcap D$ & $C^{\mathcal{I}} \cap D^{\mathcal{I}}$ \\
\hline $\begin{array}{c}\text { existential } \\
\text { restriction }\end{array}$ & $\exists r . C$ & $\begin{array}{c}\left\{x \in \Delta^{\mathcal{I}} \mid \exists y \in \Delta^{\mathcal{I}}:\right. \\
\left.(x, y) \in r^{\mathcal{I}} \wedge y \in C^{\mathcal{I}}\right\}\end{array}$ \\
\hline \hline GCI & $C \sqsubseteq D$ & $C^{\mathcal{I}} \subseteq D^{\mathcal{I}}$ \\
\hline equivalence axioms & $C \equiv D$ & $C^{\mathcal{I}}=D^{\mathcal{I}}$ \\
\hline RI & $r_{1} \circ \ldots \circ r_{k} \sqsubseteq r$ & $r_{1}^{\mathcal{I}} \circ \ldots \circ r_{k}^{\mathcal{I}} \subseteq r^{\mathcal{I}}$ \\
\hline
\end{tabular}

Table 2.1: The $\mathcal{E} \mathcal{L}$ family - Syntax and Semantics.

and worksOn are atomic relations and john and semanticweb101 are individuals. The TBox contains three subsumption axioms, related to concepts UndergraduateCourse, GraduateCourse and Professor and two concept definitions (equivalence axioms) for concepts Teacher and Researcher. In natural language, the terminological axioms can be read as follows. Undergraduate course and graduate course are types of courses. A professor is someone who teaches some undergraduate or graduate course and works on a research project. However, not everyone who works on a research project and teaches such courses is a professor, therefore only the subsumption relation is used. Further, teacher is defined as someone who teaches some course and a researcher is someone who works on some research project.

The ABox contains three assertions, two which represent concept assertions, namely that john is a professor and that semanticweb101 is a course. Further, the ABox also contains a role assertion which states that john teaches the semanticweb101 course.

As mentioned in the previous section, ontologies can be specified using description logics. In this case, concepts, relations, instances and axioms in ontologies map to concepts, roles, individuals and axioms in description logics, respectively.

There are different variants of description logics depending on which kind of logical constructors they allow. The supported logical constructors in a language have direct consequences on the properties of the language such as decidability, termination and completeness of reasoning. In this work we focus on two variants, the $\mathcal{E} \mathcal{L}$ family and $\mathcal{A L C}$.

\subsection{1 $\mathcal{E} \mathcal{L}$ family}

The $\mathcal{E} \mathcal{L}$ family of description logics includes three variants: $\mathcal{E} \mathcal{L}, \mathcal{E} \mathcal{L}^{+}$and $\mathcal{E} \mathcal{L}^{++}$. For the description logics $\mathcal{E} \mathcal{L}$ and $\mathcal{E} \mathcal{L}^{+}$the concept constructors are the top concept $T$, conjunction, and existential restriction. For $\mathcal{E L}^{++}$, we 


\begin{tabular}{|c|c|c|}
\hline Name & Syntax & Semantics \\
\hline \hline top & $\top$ & $\Delta^{\mathcal{I}}$ \\
\hline bottom & $\perp$ & $\emptyset$ \\
\hline conjunction & $C \sqcap D$ & $C^{\mathcal{I}} \cap D^{\mathcal{I}}$ \\
\hline disjunction & $C \sqcup D$ & $C^{\mathcal{I}} \cup D^{\mathcal{I}}$ \\
\hline concept negation & $\neg C$ & $\Delta^{\mathcal{I}} \backslash C^{\mathcal{I}}$ \\
\hline $\begin{array}{c}\text { existential } \\
\text { restriction }\end{array}$ & $\exists r . C$ & $\begin{array}{c}\left\{x \in \Delta^{\mathcal{I}} \mid \exists y \in \Delta^{\mathcal{I}}:\right. \\
\left.(x, y) \in r^{\mathcal{I}} \wedge y \in C^{\mathcal{I}}\right\}\end{array}$ \\
\hline universal & $\forall r . C$ & $\begin{array}{c}\left\{x \in \Delta^{\mathcal{I}} \mid \forall y \in \Delta^{\mathcal{I}}:\right. \\
\left.(x, y) \in r^{\mathcal{I}} \rightarrow y \in C^{\mathcal{I}}\right\}\end{array}$ \\
\hline restriction & & $C^{\mathcal{I}} \subseteq D^{\mathcal{I}}$ \\
\hline GCI & $C \sqsubseteq D$ & $C^{\mathcal{I}}=D^{\mathcal{I}}$ \\
\hline
\end{tabular}

Table 2.2: $\mathcal{A L C}$ - Syntax and Semantics.

have additionally the bottom concept $\perp$, nominals, and a restricted form of concrete domains. In this thesis, we consider the version of $\mathcal{E L}^{++}$without concrete domains. For the syntax and semantics of the different constructors see Table 2.1 .

In $\mathcal{E} \mathcal{L}$, a TBox can contain two types of axioms: general concept inclusions of the form $C \sqsubseteq D$ (where $C$ and $D$ are $\mathcal{E} \mathcal{L}$ concepts) and equivalence axioms the form $C \equiv D$. An equivalence axiom $C \equiv D$ can also be represented with two GCIs $C \sqsubseteq D$ and $D \sqsubseteq C$.

In the case of $\mathcal{E L}^{+}$and $\mathcal{E L}^{++}$, TBoxes may also contain role inclusions (RIs) of the form $r_{1} \circ \ldots \circ r_{m} \sqsubseteq s$ (where $r_{i}$ and $s$ are role names).

\subsection{2 $\quad \mathcal{A L C}$}

Description logic $\mathcal{A L C}$ was introduced in [88]. The logical constructors in $\mathcal{A L C}$ are concept conjunction, disjunction, negation, universal quantification. In the general case, description logic $\mathcal{A L C}$ allows general concept inclusions of the form $C \sqsubseteq D$ where $C$ and $D$ are $\mathcal{A L C}$ concepts. The syntax and semantics of the logical constructors in $\mathcal{A L C}$ are given in Table 2.2.

In this thesis we consider ontologies that can be represented by a TBox that is an acyclic terminology. An acyclic terminology is a finite set of concept definitions i.e. equivalence axioms of the form $C \equiv D$ where $C$ is an atomic concept, that neither contains multiple definitions nor cyclic definitions. A cyclic definition is a definition which defines concepts in terms of themselves or in terms of concepts that indirectly refer to them [15]. 


\subsection{Reasoning in description logics}

Knowledge bases usually contain implicit knowledge not explicitly defined using terminological or assertional axioms. In the example in Figure 2.1 it is easy to see that Professor is a Researcher given that he/she works on a ResearchProject, as a consequence john is also an instance of concept Researcher. However this knowledge is not explicitly defined in the knowledge base. In order to infer this implicit knowledge, knowledge representation systems based on description logics enable a number of reasoning tasks.

Reasoning tasks in description logics can be divided into two categories: reasoning tasks for concepts and reasoning tasks for ABoxes [15]. Reasoning tasks for concepts include checking [15]:

- Satisfiability - a concept $\mathrm{C}$ is satisfiable w.r.t. a TBox $\mathrm{T}$ if there exists a model $\mathcal{I}$ of $\mathcal{T}$ such that $C^{\mathcal{I}}$ is non-empty. A TBox is said to be incoherent if it contains an unsatisfiable concept.

- Subsumption - a concept $\mathrm{C}$ is subsumed by D w.r.t. a TBox $\mathcal{T}$ if $C^{\mathcal{I}} \subseteq D^{\mathcal{I}}$ holds in every model $\mathcal{I}$ of $\mathcal{T}$. This can also be written as $\mathcal{T} \models C \sqsubseteq D$.

- Equivalence - a concept $\mathrm{C}$ is equivalent to $\mathrm{D}$ w.r.t. a TBox $\mathcal{T}$ if $C^{\mathcal{I}}=D^{\mathcal{I}}$ holds in every model $\mathcal{I}$ of $\mathcal{T}$.

- Disjointness - a concept $\mathrm{C}$ is disjoint from concept D w.r.t. a TBox $\mathcal{T}$ if $C^{\mathcal{I}} \cap D^{\mathcal{I}}=\emptyset$ holds in every model $\mathcal{I}$ of $\mathcal{T}$.

Reasoning tasks for ABoxes include the following tasks [15]:

- Instance checking - checking if an assertion $\alpha$ is entailed by an ABox $\mathcal{A}(\mathcal{A} \models \alpha)$, i.e. that every model of $\mathcal{A}$ is also a model of $\alpha$.

- Realization - given an individual $a$ and a set of concepts, the task is to identify the most specific concepts $\mathrm{C}$ such that $\mathcal{A} \models C(a)$ where the most specific concepts are those which are minimal w.r.t. the subsumption ordering.

- Retrieval - represents retrieval of all individuals of some concept, i.e. for a given concept $C$ the idea is to identify all $a$ such that $\mathcal{A}=C(a)$.

- Knowledge base consistency - a knowledge base is consistent if there exists an interpretation $\mathcal{I}$ such that satisfies both $\mathcal{T}$ and $\mathcal{A}$.

The reasoning tasks are closely related and can often be reduced from one to the other. For example, a concept $\mathrm{C}$ is subsumed by $\mathrm{D}$ if $\mathrm{C} \sqcap \neg \mathrm{D}$ is unsatisfiable. Given this, reasoning algorithms usually provide means for solving only one reasoning task, while the others are solved by reduction to it. 


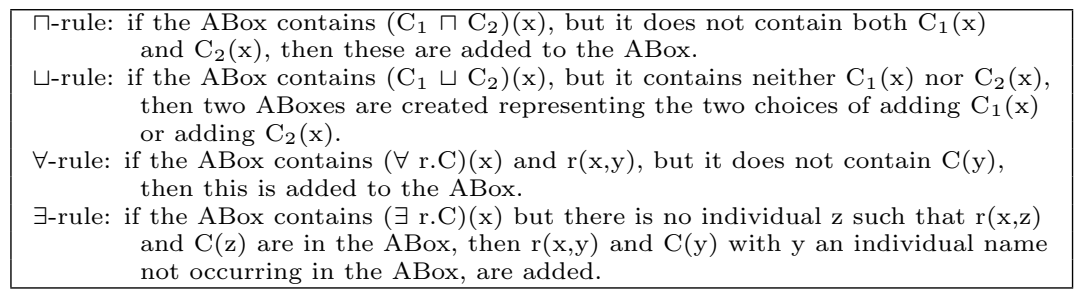

Figure 2.2: Transformation rules (e.g. [16]).

There are a number of reasoning algorithms for description logics and in the following section we introduce tableaux reasoning algorithm which will be used in Chapter 5 .

\subsubsection{Tableaux reasoning}

Checking satisfiability of concepts in ontologies represented in the studied description logics can be done using a tableau-based algorithm (e.g. [16]). To test whether a concept $\mathrm{C}$ is satisfiable such an algorithm starts with an ABox containing the statement $\mathrm{C}(\mathrm{x})^{1}$ where $\mathrm{x}$ is a new individual. It is usually assumed that $\mathrm{C}$ is normalized to negation normal form i.e. negations can only appear in front of atomic concepts. This is done by applying De Morgan's laws and rules for quantifiers. For example, the negation normal form of $\neg(C \sqcup \exists r . D)$ would be $\neg C \sqcap \forall r . \neg D$. Next, consistency-preserving transformation rules are applied to the ABox. The Figure 2.2 lists the rules for description logic $\mathcal{A L C}$. The $\sqcap-, \forall$ - and $\exists$-rules extend the ABox while the $\sqcup$-rule creates multiple ABoxes representing different choices for the disjunction. The algorithm continues applying these transformation rules to the ABox until no more rules apply. This process is called completion and if one of the final ABoxes does not contain a contradiction - clash (we say that it is open), then satisfiability is proven, otherwise unsatisfiability is proven.

One way of implementing this approach is through completion graphs which are directed graphs in which every node represents an ABox. Application of the $\sqcup$-rule produces new nodes with one statement each, while the other rules add statements to the node on which the rule is applied. The ABox for a node contains all the statements of the node as well as the statements of the nodes on the path to the root. Satisfiability is proven if at least one of the ABoxes connected to a leaf node does not contain a contradiction, otherwise unsatisfiability is proven.

In order to take into account subsumption axioms and concept definitions in the TBox, ABoxes have to be expanded with statements of the form $x: \neg C \sqcup D$ for every individual $x$ in the ABox for each axiom $C \sqsubseteq D$ in

\footnotetext{
${ }^{1}$ These statements are also written as $x: C$.
} 


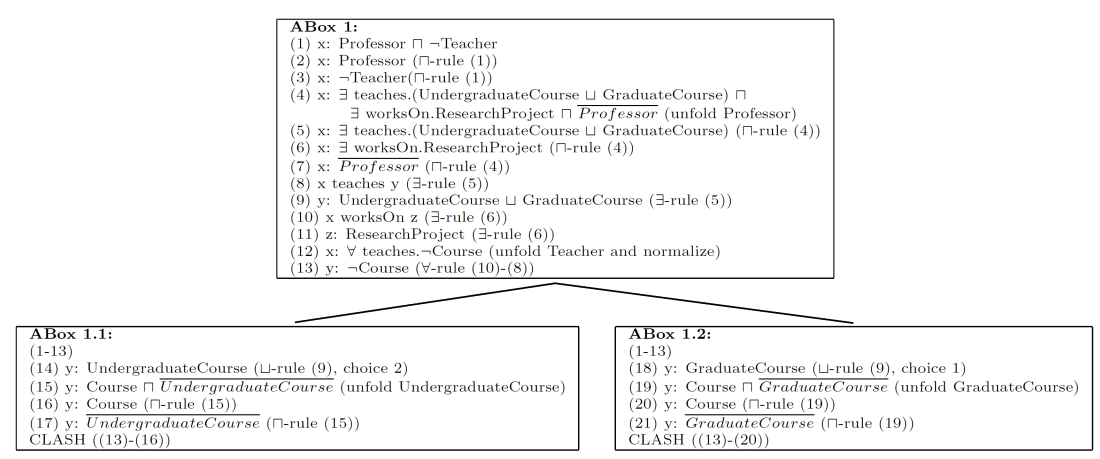

Figure 2.3: Completion graph for Professor $\sqcap \neg$ Teacher.

the TBox. This is often a costly task and different methods are used to minimize the need for such expansions.

In this thesis we assume that an ontology is represented by a knowledge base containing a TBox that is an acyclic terminology and an empty ABox. In this case reasoning can be reduced to reasoning without the TBox by unfolding the definitions. However, for efficiency reasons, instead of running the previously described satisfiability checking algorithm on an unfolded concept description, the unfolding is usually performed on demand within the satisifiability algorithm. When dealing with acyclic TBoxes concept definitions are unfolded on demand as follows:

- if the TBox contains an axiom of the form $A \equiv B$ and an ABox contains a statement $x: A$ then statement $x: B$ is also added to the ABox.

- if the TBox contains an axiom of the form $A \sqsubseteq B$ and an ABox contains a statement $x: A$ then statements $x: B$ and $x: \bar{A}$ where $\bar{A}$ represents a new concept name are also added to the ABox.

It has been proven that satisfiability checking w.r.t. acyclic terminologies is PSPACE-complete in $\mathcal{A L C}$ [76].

Figure 2.3 shows a completion graph for subsumption checking of relation Professor $\sqsubseteq$ Teacher with respect to the knowledge base in Figure 2.1. As explained earlier, the subsumption check can be reduced to a satisfiability check. Therefore, in order to prove that Professor $\sqsubseteq$ Teacher holds it is necessary to prove that Professor $\sqcap \neg$ Teacher is unsatisfiable on an empty ABox meaning that all leaf ABoxes contain a contradiction. The algorithm starts with the statement $x$ : Professor $\sqcap \neg$ Teacher where $\mathrm{x}$ is a new individual. We continue by unfolding and applying $\sqcap$-, $\forall$ - and $\exists$-rules until no more unfoldings are possible and no more rules apply. In the completion graph in Figure 2.3 this is represented by steps (1) to (13) in ABox 1. Next, we apply a $\sqcup$-rule which produces two new ABoxes containing statements 
from the initial ABox together with statements representing different choices for the disjunction (statements (14) and (18)). The algorithm continues applying transformation rules and after adding statement (17) in ABox 1.1 a clash is detected given that $y$ is of type Course and $\neg$ Course at the same time. The same clash is detected in ABox 1.2. Given that all leaf ABoxes are closed the subsumption is proven.

\subsection{Debugging ontologies}

With the increasing presence of data sources on the Internet more and more research effort is put into finding possible ways for integrating and searching such often heterogeneous sources. Semantic Web technologies such as ontologies are becoming a key technology in this effort. As exemplified in Chapter 1 high quality ontologies are important for acquiring reliable results in semantically-enabled applications. However, developing and maintaining ontologies is a difficult task and it is often the case that defects are introduced into ontologies, both in the development phase and with future updates. One of the reasons for this is that domain experts who usually develop ontologies, lack expertise when it comes to knowledge representation paradigms as well as good and bad practices for developing ontologies. As a result defects ranging from simple syntactic errors to wrong use of language constructs are introduced into ontologies. For example, ontology developers often mistake relation part-of for the is-a relation. Another example of defect is a situation in which domain experts introduce logical contradictions into the ontology.

In order to acquire high quality ontologies it is necessary to resolve these kind of defects which is the focus of ontology debugging. Ontology debugging can be divided into two phases i.e. detection phase and repairing phase. In the detection phase, ontology defects are detected using various techniques. The complexity of the detection phase differs with types of defects.

In the repairing phase, the detected defects are repaired. Depending on which kind of defects are debugged different approaches are used. For example, the idea when dealing with missing relations is to add knowledge to the ontology which would make the missing relations derivable. A method for dealing with wrong relations is to remove relations which make the wrong relations derivable.

In the recent years there has been a growing research interest in the area of ontology debugging which led to the founding of the International Workshop on Debugging Ontologies and Ontology Mappings which provides a venue for discussing ontology debugging methods and techniques. 


\subsubsection{Classification of defects}

There are three types of defects according to [57]:

- syntactic defects - represent syntactic errors, for example missing tags or incorrect format. This kind of defects is easy to detect and can be resolved using parsers and validators.

- semantic defects - these defects can be further classified into:

- unsatisfiable concepts - concepts to which no instance can belong, i.e. concepts which are equivalent to $\perp$. For example let us consider an ontology with the following axioms:

$$
\begin{aligned}
& \text { Bird } \sqsubseteq \text { FlyingAnimal } \\
& \text { Penguin } \sqsubseteq \text { Bird } \sqcap \neg \text { FlyingAnimal }
\end{aligned}
$$

In this case concept Penguin is defined as a subconcept of Bird and a flightless animal ( $\neg$ FlyingAnimal). However, given that concept Bird is defined as a subconcept of FlyingAnimal it follows that Penguin is also a subconcept of FlyingAnimal. So in this case Penguin is at the same time a $\neg$ FlyingAnimal and a FlyingAnimal which would mean that Penguin is equivalent to $\perp$ and therefore an unsatisfiable concept.

- incoherent ontologies - ontologies which contain an unsatisfiable concept. Therefore, the ontology from the previous example would be an incoherent ontology given that it contains the unsatisfiable concept Penguin.

- inconsistent ontologies - ontologies which contain a contradiction, e.g. an instance of an unsatisfiable concept or an ontology from which it is possible to derive that $\perp \equiv \top$. In our case, if we added an instance of concept Penguin to the ontology from the example it would be inconsistent.

As introduced in Section 2.3 one of the reasoning tasks in ontologies is satisfiability checking which can be used to detect this kind of defects. However, the repairing phase is not trivial and there are a number of different approaches for dealing with this kind of defects (see Chapter 6).

- modeling defects - represent defects which are a result of modeling errors. An example of this kind of defects are missing or wrong is-a relations. This kind of defects requires domain knowledge to detect and resolve. In Figure 1.1 missing is-a relations are wrist joint $\sqsubseteq$ joint, hip joint $\sqsubseteq$ joint, knee joint $\sqsubseteq$ joint, elbow joint $\sqsubseteq$ joint, shoulder joint $\sqsubseteq$ joint, ankle joint $\sqsubseteq$ joint and metacarpo-phalangeal joint $\sqsubseteq$ joint. 


\subsection{Abduction in description logics}

Logical abductive reasoning is a type of inference. The task of abductive reasoning is given a set of formulas (theory $\mathrm{T}$ ) and a formula which represents an observation (an abductive query $\mathrm{O}$ ) to find a set of formulas (an explanation $\mathrm{E}$ ) such that $\mathrm{T} \cup \mathrm{E}$ is consistent and $\mathrm{T} \cup \mathrm{E} \models \mathrm{O}$. In some definitions, logic-based abduction also includes a set of formulas $\mathrm{H}$ called hypotheses from which explanations are formed. When it comes to abductive reasoning in description logics Elsenbroich et al. [38] defined the following categories of abductive reasoning:

- ABox abduction - retrieving abductively concept or role instances which together with the knowledge base would entail a given ABox assertion.

- Concept abduction - finding abductively concepts which are subsumed by a given concept $\mathrm{C}$.

- TBox abduction - retrieving abductively relations which together with the knowledge base entail a given relation $\mathrm{C} \sqsubseteq \mathrm{D}$.

- Knowledge-base abduction - retrieving abductively a set of TBox and ABox assertions which together with the knowledge base entail an abductive query which can be either an ABox or TBox assertion.

In this thesis we focus on TBox abduction which is defined in [38] as follows.

Definition 1 (TBox Abduction) Let $\mathcal{L}$ be a description logic, $\Gamma$ a knowledge base in $\mathcal{L}$, and $A, B$ concepts that are satisfiable w.r.t. to $\Gamma$ and such that $\Gamma \cup\{A \sqsubseteq B\}$ is consistent. A solution to the TBox abduction problem for $(\Gamma, A, B)$ is any finite set $S=\left\{E_{i} \sqsubseteq F_{i} \mid i \leq n\right\}$ of TBox assertions, such that $T \cup S$ is consistent and $T \cup S \models A \sqsubseteq B$. The set of all such solutions is denoted as $\mathcal{S}_{T}(\Gamma, A, B)$.

\subsubsection{Constraints on solutions}

Eiter and Gottlob [37] showed that computing all abductive solutions even in propositional logic is not in all cases possible or practical. Therefore, constraining solutions can significantly reduce the search space and allow practical use of logical-based abduction. Examples of constraints on solutions are subset minimality and minimum cardinality. A solution $S$ is said to be subset minimal if no proper subset of $S$ is a solution. In the case of minimum cardinality, solutions containing fewer formulae are preferred.

There are a number of restrictions which can be imposed on solutions of abductive problems in description logics. One such restriction is consistency, meaning that the union of the background theory (knowledge base) and solution to the abduction problem should be consistent, e.g. $\top \equiv \perp$ does 
not hold in the knowledge base. However, Elsenbroich et al. [38] argue that inconsistent solutions can be valuable as they could imply the need for a revision of a knowledge base. Other restrictions such as relevance and minimality can be used for restricting trivial solutions. Relevant solutions are those solutions which do not directly entail the abductive query. In other words, an abductive query needs to be a logical consequence of a union of a solution and a knowledge base and not only the solution. Elsenbroich et al. distinguish between two types of minimality, syntactic in which case a solution has to be minimal length and semantic ${ }^{2}$ in which case a solution should only contain information which is necessary to make an abductive query a logical consequence of a knowledge base and a solution. For example, if $\mathrm{A}$ is found to be a solution to some abductive query then $\mathrm{A} \cap \mathrm{B}$ is not a semantically minimal solution as it contains B which is extra information.

\footnotetext{
${ }^{2}$ This preference criterion is not directly related to semantic maximality later discussed in this work.
} 


\section{Chapter 3}

\section{Repairing incomplete ontologies - framework}

This chapter ${ }^{1}$ presents our framework for repairing missing is-a structure in ontologies. As discussed in Section 1.1, existing detection methods usually do not find all missing is-a relations so there exist more interesting approaches for repairing missing is-a structure other than just adding the missing is-a relations. We have also shown that these other repairing approaches can introduce new knowledge to the ontology which was not previously detected by the detection algorithm. In our example in Figure 1.1 the missing is-a structure could be repaired by adding limb-joint $\sqsubseteq$ joint which represents new knowledge which was not derivable from the ontology and not originally detected by the detection algorithm. Further, resolving this type of defects requires a domain expert to validate the logical solutions as not all logical solutions are correct according to the domain.

The TBox abduction problem defined in [38] formalizes the problem of repairing a single is-a relation, i.e. identifying a set of relations which need to be added to an ontology so that the missing is-a relation is derivable and the extended ontology is consistent. Our framework for repairing missing is-a structure extends the TBox abduction problem by considering the set of missing is-a relations as well as formalizing the role of a domain expert which is needed for validating logical solutions.

This chapter is organized as follows, in Section 3.1 we formalize the problem of repairing missing is-a structure as a generalized version of the TBox abduction problem. We also define different properties for the ontology, the set of is-a relations to repair, and the domain expert and discuss the influences of these properties on the existence of solutions for the abduction problem. In general, when solutions exist, there may be many solutions. As not all solutions are equally interesting, in Section 3.2 we propose two

\footnotetext{
${ }^{1}$ The chapter is a refined version of [72]
} 
preference criteria on the solutions as well as different ways to combine these. Further, in Section 3.3 we discuss the consequences of our analyses for debugging in practice.

\subsection{Abduction Framework}

In the following we explain how the problem of finding possible ways to repair the missing is-a structure in an ontology is formalized as a generalized version of the TBox abduction problem (extension of [67]). We assume that our ontology is represented using a TBox $T$. The identified is-a relations to repair are then represented by a set $M$ of atomic concept subsumptions. As discussed, $M$ usually does not contain all missing is-a relations. To repair the ontology, it should be extended with a set $S$ of atomic concept subsumptions (repair) such that the extended ontology is consistent and the missing is-a relations are derivable from the extended ontology. However, the added atomic concept subsumptions should be correct according to the domain $^{2}$. In general, the set of all atomic concept subsumptions that are correct according to the domain are not known beforehand. Indeed, if this set were given then we would only have to add this to the ontology. The common case, however, is that we do not have this set, but instead can rely on a domain expert that can decide whether an atomic concept subsumption is correct according to the domain. In our formalization the domain expert is represented by an oracle function $O r$ that when given an atomic concept subsumption, returns true or false. It is then required that for every atomic concept subsumption $s \in S$, we have that $\operatorname{Or}(s)=$ true. The following definition formalizes this.

Definition 2 (Generalized TBox Abduction) Let $T$ be a TBox in language $\mathcal{L}$ and $C$ be the set of all atomic concepts in $T$. Let $M=\left\{A_{i} \sqsubseteq B_{i}\right\}_{i=1}^{n}$ with $A_{i}, B_{i} \in C$ be a finite set of TBox assertions. Let $O r:\left\{C_{i} \sqsubseteq D_{i} \mid\right.$ $\left.C_{i}, D_{i} \in C\right\} \rightarrow\{$ true, false $\}$. A solution to the generalized TBox abduction problem (GTAP) $(T, C, O r, M)$ is any finite set of TBox assertions $S=\left\{E_{i} \sqsubseteq F_{i}\right\}_{i=1}^{k}$ such that $\forall E_{i}, F_{i}: E_{i}, F_{i} \in C, \forall E_{i}, F_{i}: \operatorname{Or}\left(E_{i} \sqsubseteq F_{i}\right)=$ true, $T \cup S$ is consistent and $T \cup S \models M$. The set of all such solutions is denoted as $\mathcal{S}(T, C, O r, M)$.

Next, we discuss different properties of $T, O r$ and $M$ and how these properties and their combinations affect the existence and type of solutions. In this discussion we make the assumption that the domain is consistent.

We note that if $T$ is not consistent then there are no solutions satisfying the definition (as $T \cup S$ would be inconsistent). If $T$ is not consistent, it means that the original ontology is not consistent. In this case approaches for debugging semantic defects could be used to obtain a consistent ontology.

\footnotetext{
${ }^{2}$ In the remainder of this thesis when we say that concept subsumptions or is-a relations are correct, we mean correct according to the domain.
} 
However, even if $T$ is consistent, it is possible that $T$ contains relations which are not correct. It would mean that the developers introduced a modeling defect. Therefore, we identify two cases for $T$ - all the is-a relations in $T$ are correct ('T correct' in Table 3.1), or not ('T not correct' in Table 3.1).

For $M$ there are two cases. In the first case we assume that all is-a relations in $M$ are correct, and thus they are really missing is-a relations ('Missing' in Table 3.1). In the second case $M$ may contain missing as well as wrong is-a relations ('Missing + Wrong' in Table 3.1). This is a common case when possible missing is-a relations are generated by detection algorithms (e.g., using patterns or ontology learning methods) and not validated by a domain expert. It may also occur when $M$ is generated by domain experts (e.g., using inspection) - as it is an error-prone task, the experts may make mistakes.

For $O r$ we identified the following interesting cases. In the first case ('Complete Knowledge' in Table 3.1) Or returns true to all correct is-a relations and no others. In this case we are sure that if $O r$ returns true, that an is-a relation is correct and if not, it is not correct. This case represents the ideal situation of an all-knowing domain expert. In the second case ('Partial-Correct' in Table 3.1) Or returns true for correct is-a relations, but not necessarily all. This case represents a domain expert who knows a part of the domain well. If the domain expert validates an is-a relation as correct, it is correct. Otherwise, the is-a relation is wrong or the domain expert does not know. An approximation of this case is when there are several domain experts who may have different opinions and we use a sceptical approach. We only consider an is-a relation correct if all domain experts validate it as correct. In the third case ('Wrong' in Table 3.1) Or may return true for relations that are not correct. In this case, the domain expert can make mistakes regarding the validation of is-a relations. Some wrong is-a relations may be validated as correct. This is a common case as exemplified by the use case in [55] where experts initially validated a relation as correct. However, further inspection showed that the definitions of two concepts are incompatible and the relation was changed into wrong. The fourth and fifth cases represent situations where there is no domain expert. In the fourth case all possible is-a relations are validated to be correct and thus $\forall \mathrm{E}, \mathrm{F}$ $\in \mathrm{C}: \operatorname{Or}(\mathrm{E} \sqsubseteq \mathrm{F})=$ true ('No Expert' in Table 3.1). In the fifth case (not in the table) no is-a relation is validated to be correct and thus $\forall \mathrm{E}, \mathrm{F} \in \mathrm{C}$ : $\operatorname{Or}(\mathrm{E} \sqsubseteq \mathrm{F})=$ false. For the fifth case there can be only one solution, i.e., $\mathrm{S}$ $=\emptyset$ and this only in the case where $T \models M$ (and thus the is-a relations in $M$ were not actually missing).

In our example in Figure 3.1 which is based on the ontology in Figure 1.1, $\mathrm{Or}_{1}, \mathrm{Or}_{2}, \mathrm{Or}_{3}$ and $\mathrm{Or}_{4}$ are examples of 'Complete Knowledge', 'PartialCorrect', 'Wrong' and 'No expert', respectively.

Table 3.1 shows the properties for $T, O r, M$ and their combinations. For each combination we give information about the relationship between $M$ and $O r$, the existence of solutions and the correctness of the solutions. 


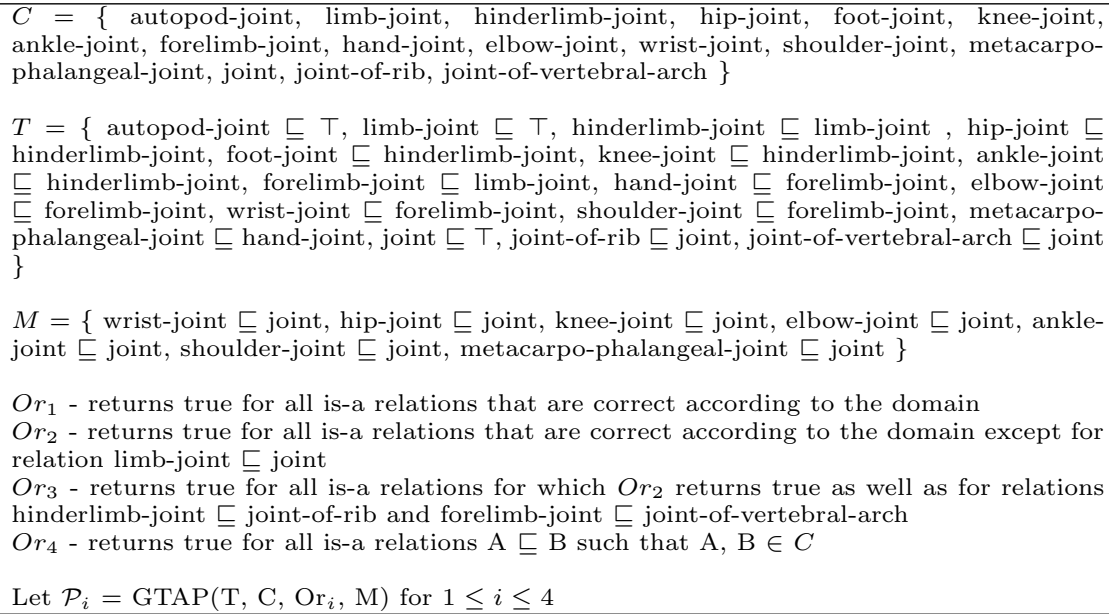

Figure 3.1: Small example based on the ontology from Figure 1.1.

Here, we summarize the findings.

An ideal situation is the case where the domain expert has complete knowledge ( $\mathrm{Or}$ returns true for all correct is-a relations and no others) and $T$ and $M$ contain only correct is-a relations. In this case, it holds that $\forall \mathrm{m}$ $\in \mathrm{M}: \operatorname{Or}(\mathrm{m})=$ true as the domain expert has complete knowledge. Further, $M$ is a solution and all solutions are correct.

For any case where $T \cup M$ is inconsistent, there is no solution. Indeed, for any solution $S$ we have that $T \cup S \models M$ and thus $T \cup S$ would not be consistent.

In the cases where $M$ contains wrong is-a relations, there may be no solutions. If there are solutions, these are not correct. Further, correctness of solutions is only guaranteed when $M$ does not contain wrong is-a relations and $O r$ represents complete knowledge or partial-correct.

There are no solutions if $T \cup S$ is inconsistent for every non-empty solution S.

If $\forall \mathrm{m} \in \mathrm{M}: \operatorname{Or}(\mathrm{m})=$ true and $T \cup M$ is consistent, then $M$ is a solution.

In the case of no expert $(\forall \mathrm{E}, \mathrm{F} \in \mathrm{C}: \operatorname{Or}(\mathrm{E} \sqsubseteq \mathrm{F})=$ true $)$ we have that $\forall \mathrm{m} \in \mathrm{M}: \operatorname{Or}(\mathrm{m})=$ true and all is-a relations are allowed in the solution. Therefore, if $T \cup M$ is consistent, then $M$ is a solution, otherwise there is no solution. However, as there is no domain expert, there is no guarantee that any solution other than $M$ is correct. Further, in the cases where $M$ contains wrong is-a relations, $M$ is a solution, but not correct. As there is no validation, only logical consistency can be guaranteed, but no correctness. 


\begin{tabular}{|c|c|c|}
\hline M & \multicolumn{2}{|c|}{ Missing } \\
\hline Or & $\mathrm{T}$ correct & T not correct \\
\hline $\begin{array}{l}\text { Complete } \\
\text { Knowledge }\end{array}$ & $\begin{array}{l}\forall \mathrm{m} \in \mathrm{M}: \mathrm{Or}(\mathrm{m})=\text { true } \\
M \text { is solution } \\
\text { All solutions are correct }\end{array}$ & $\begin{array}{l}\forall \mathrm{m} \in \mathrm{M}: \operatorname{Or}(\mathrm{m})=\text { true } \\
\text { No solution if } T \cup M \text { inconsistent } \\
M \text { is solution iff } T \cup M \text { consistent } \\
\text { All solutions are correct }\end{array}$ \\
\hline $\begin{array}{l}\text { Partial- } \\
\text { Correct }\end{array}$ & $\begin{array}{l}\forall \mathrm{m} \in \mathrm{M}: \operatorname{Or}(\mathrm{m})=\text { true or } \\
\exists \mathrm{m} \in \mathrm{M}: \operatorname{Or}(\mathrm{m})=\text { false } \\
\text { if } \forall \mathrm{m} \in \mathrm{M}: \operatorname{Or}(\mathrm{m})=\text { true } \\
\text { then } M \text { is a solution } \\
\text { All solutions are correct }\end{array}$ & $\begin{array}{l}\forall \mathrm{m} \in \mathrm{M}: \operatorname{Or}(\mathrm{m})=\text { true or } \\
\exists \mathrm{m} \in \mathrm{M}: \operatorname{Or}(\mathrm{m})=\text { false } \\
\text { No solution if } T \cup M \text { inconsistent } \\
\text { No solution if } \\
\quad \forall S: S \neq \emptyset \wedge\left\{E_{i} \sqsubseteq F_{i} \mid E_{i} \sqsubseteq F_{i} \in S \wedge \operatorname{Or}\left(E_{i} \sqsubseteq F_{i}\right)=\text { true }\right\} \\
\quad \rightarrow T \cup S \text { inconsistent } \\
\text { if } T \cup M \text { consistent } \wedge \forall \mathrm{m} \in \mathrm{M}: \operatorname{Or}(\mathrm{m})=\text { true } \\
\quad \text { then } M \text { is a solution } \\
\text { All solutions are correct }\end{array}$ \\
\hline Wrong & $\begin{array}{l}\forall \mathrm{m} \in \mathrm{M}: \operatorname{Or}(\mathrm{m})=\text { true or } \\
\exists \mathrm{m} \in \mathrm{M}: \operatorname{Or}(\mathrm{m})=\text { false } \\
\text { No solution if } \\
\quad \forall S: S \neq \emptyset \wedge\left\{E_{i} \sqsubseteq F_{i} \mid E_{i} \sqsubseteq F_{i} \in S \wedge \operatorname{Or}\left(E_{i} \sqsubseteq F_{i}\right)=\text { true }\right\} \\
\quad \rightarrow T \cup S \text { inconsistent } \\
\text { if } \forall \mathrm{m} \in \mathrm{M}: \operatorname{Or}(\mathrm{m})=\text { true } \\
\quad \text { then } M \text { is a solution } \\
\text { If } M \text { is solution, then correct, no guarantee otherwise }\end{array}$ & $\begin{array}{l}\forall \mathrm{m} \in \mathrm{M}: \operatorname{Or}(\mathrm{m})=\text { true or } \\
\exists \mathrm{m} \in \mathrm{M}: \operatorname{Or}(\mathrm{m})=\text { false } \\
\text { No solution if } T \cup M \text { inconsistent } \\
\text { No solution if } \\
\quad \forall S: S \neq \emptyset \wedge\left\{E_{i} \sqsubseteq F_{i} \mid E_{i} \sqsubseteq F_{i} \in S \wedge \operatorname{Or}\left(E_{i} \sqsubseteq F_{i}\right)=\text { true }\right\} \\
\quad \rightarrow T \cup S \text { inconsistent } \\
\text { if } T \cup M \text { consistent } \wedge \forall \mathrm{m} \in \mathrm{M}: \operatorname{Or}(\mathrm{m})=\text { true } \\
\quad \text { then } M \text { is a solution } \\
\text { If } M \text { is solution, then correct (but not } T \cup M), \\
\text { no guarantee otherwise }\end{array}$ \\
\hline $\begin{array}{l}\text { No } \\
\text { Expert }\end{array}$ & $\begin{array}{l}\forall \mathrm{m} \in \mathrm{M}: \operatorname{Or}(\mathrm{m})=\text { true } \\
M \text { is solution } \\
\text { If } M \text { is solution, then correct, no guarantee otherwise }\end{array}$ & $\begin{array}{l}\forall \mathrm{m} \in \mathrm{M}: \operatorname{Or}(\mathrm{m})=\operatorname{true} \\
M \text { is solution iff } T \cup M \text { consistent } \\
\text { If } M \text { is solution, then correct (but not } T \cup M \text { ), } \\
\text { no guarantee otherwise }\end{array}$ \\
\hline & $\mathrm{T}$ correct & T not correct \\
\hline $\begin{array}{l}\text { Complete } \\
\text { Knowledge }\end{array}$ & $\begin{array}{l}\exists \mathrm{m} \in \mathrm{M}: \operatorname{Or}(\mathrm{m})=\text { false } \\
\text { No solution }\end{array}$ & $\begin{array}{l}\exists \mathrm{m} \in \mathrm{M}: \operatorname{Or}(\mathrm{m})=\text { false } \\
\text { No solution if } T \cup M \text { inconsistent } \\
\text { No solution if } \\
\quad \forall S: S \neq \emptyset \wedge\left\{E_{i} \sqsubseteq F_{i} \mid E_{i} \sqsubseteq F_{i} \in S \wedge \operatorname{Or}\left(E_{i} \sqsubseteq F_{i}\right)=\text { true }\right\} \\
\quad \rightarrow T \cup S \text { inconsistent } \\
\text { The solutions are not correct }\end{array}$ \\
\hline $\begin{array}{l}\text { Partial- } \\
\text { Correct }\end{array}$ & $\begin{array}{l}\exists \mathrm{m} \in \mathrm{M}: \mathrm{Or}(\mathrm{m})=\text { false } \\
\text { No solution }\end{array}$ & $\begin{array}{l}\exists \mathrm{m} \in \mathrm{M}: \operatorname{Or}(\mathrm{m})=\text { false } \\
\text { No solution if } T \cup M \text { inconsistent } \\
\text { No solution if } \\
\quad \forall S: S \neq \emptyset \wedge\left\{E_{i} \sqsubseteq F_{i} \mid E_{i} \sqsubseteq F_{i} \in S \wedge \operatorname{Or}\left(E_{i} \sqsubseteq F_{i}\right)=\text { true }\right\} \\
\quad \rightarrow T \cup S \text { inconsistent } \\
\text { The solutions are not correct }\end{array}$ \\
\hline Wrong & $\begin{array}{l}\forall \mathrm{m} \in \mathrm{M}: \operatorname{Or}(\mathrm{m})=\text { true or } \\
\exists \mathrm{m} \in \mathrm{M}: \operatorname{Or}(\mathrm{m})=\text { false } \\
\text { No solution if } T \cup M \text { inconsistent } \\
\text { No solution if } \\
\quad \forall S: S \neq \emptyset \wedge\left\{E_{i} \sqsubseteq F_{i} \mid E_{i} \sqsubseteq F_{i} \in S \wedge \operatorname{Or}\left(E_{i} \sqsubseteq F_{i}\right)=\text { true }\right\} \\
\quad \vec{T} \cup S \text { inconsistent } \\
\text { if } T \cup M \text { consistent } \wedge \forall \mathrm{m} \in \mathrm{M}: \operatorname{Or}(\mathrm{m})=\text { true } \\
\quad \text { then } M \text { is a solution } \\
\text { The solutions are not correct }\end{array}$ & $\begin{array}{l}\forall \mathrm{m} \in \mathrm{M}: \operatorname{Or}(\mathrm{m})=\text { true or } \\
\exists \mathrm{m} \in \mathrm{M}: \operatorname{Or}(\mathrm{m})=\text { false } \\
\text { No solution if } T \cup M \text { inconsistent } \\
\text { No solution if } \\
\quad \forall S: S \neq \emptyset \wedge\left\{E_{i} \sqsubseteq F_{i} \mid E_{i} \sqsubseteq F_{i} \in S \wedge \operatorname{Or}\left(E_{i} \sqsubseteq F_{i}\right)=\text { true }\right\} \\
\quad \vec{T} \cup S \text { inconsistent } \\
\text { if } T \cup M \text { consistent } \wedge \forall \mathrm{m} \in \mathrm{M}: \operatorname{Or}(\mathrm{m})=\text { true } \\
\quad \text { then } M \text { is a solution } \\
\text { The solutions are not correct }\end{array}$ \\
\hline $\begin{array}{l}\text { No } \\
\text { Expert }\end{array}$ & $\begin{array}{l}\forall \mathrm{m} \in \mathrm{M}: \operatorname{Or}(\mathrm{m})=\text { true } \\
M \text { is solution iff } T \cup M \text { consistent } \\
\text { The solutions are not correct }\end{array}$ & $\begin{array}{l}\forall \mathrm{m} \in \mathrm{M}: \operatorname{Or}(\mathrm{m})=\text { true } \\
M \text { is solution iff } T \cup M \text { consistent } \\
\text { The solutions are not correct }\end{array}$ \\
\hline
\end{tabular}

Table 3.1: Different combinations of cases for $T$, Or and $M$.

\subsection{Solutions with preference criteria}

There can be many solutions for a GTAP and, as explained earlier, not all solutions are equally interesting.

Ontology repairing of missing is-a relations follows different preference criteria from the logic based abduction framework, in the sense that a more informative solution is preferred to a less informative one. Note that the informativeness is a measurement for how much information the added subsumptions (i.e. solution $S$ ) can derive. See Definition 4 for the precise formulation. This is in contrast to the criteria of minimality (e.g. subset minimality, cardinality minimality) from the abduction framework. In principle this difference on the preference stems from the original purpose of the two formalisms. The abduction framework is often used for diagnostic scenarios, thus the essential goal is to confine the cause of the problem as 
small as possible. Whilst for ontology repairing, the goal is to add more subsumptions to enrich the ontology. As long as the added rules are correct, a more informative repairing means more enrichment to the ontology. However, there are technical difficulties finding the most informative solution as such. A brute-force method to create a most informative solution is to check for each pair of atomic concepts $A$ and $B$, whether $\operatorname{Or}(A \sqsubseteq B)=\operatorname{true}$ and if so, add $A \sqsubseteq B$ to the ontology. In practice, for large ontologies this is infeasible.

Definition 3 (Subset Minimality) A solution $S$ to the GTAP (T, C, Or, $M)$ is said to be subset minimal iff there is no proper subset $S^{\prime} \subsetneq S$ such that $S^{\prime}$ is a solution. The set of all subset minimal solutions is denoted as $\mathcal{S}_{\min }(T, C, O r, M)$.

Examples of subset minimal solutions for $\mathcal{P}_{1}$ in Figure 3.1 are $\{$ limb-joint $\sqsubseteq$ joint $\}$ and $\{$ hinderlimb-joint $\sqsubseteq$ joint, forelimb-joint $\sqsubseteq$ joint $\}$. Solution \{hinderlimb-joint $\sqsubseteq$ joint, forelimb-joint $\sqsubseteq$ joint $\}$ is also a subset minimal solution for $\mathcal{P}_{2}, \mathcal{P}_{3}$ and $\mathcal{P}_{4}$.

The second criterion prefers solutions that imply more information.

Definition 4 (More Informative) Let $S$ and $S^{\prime}$ be two solutions to the $\operatorname{GTAP}(T, C, O r, M)$. S is said to be more informative than $S^{\prime}$ iff $T \cup S \models S^{\prime}$ and $T \cup S^{\prime} \forall$ S. Further, we say that $S$ is equally informative as $S^{\prime}$ iff $T \cup S \models S^{\prime}$ and $T \cup S^{\prime} \models S$.

Consider two solutions to $\mathcal{P}_{1}$ in Figure $3.1, \mathrm{~S}=\{$ limb-joint $\sqsubseteq$ joint $\}$ and $\mathrm{S}^{\prime}=\{$ hinderlimb-joint $\sqsubseteq$ joint, forelimb-joint $\sqsubseteq$ joint $\} . \mathrm{S}$ is more informative than $\mathrm{S}^{\prime}$ as $\mathrm{T} \cup \mathrm{S}$ entails limb-joint $\sqsubseteq$ joint in addition to everything that $\mathrm{T}$ $\cup S^{\prime}$ entails.

Definition 5 (Semantic Maximality) A solution $S$ to the $G T A P(T, C$, Or, $M)$ is said to be semantically maximal iff there is no solution $S^{\prime}$ which is more informative than $S$. The set of all semantically maximal solutions is denoted as $\mathcal{S}^{\max }(T, C, O r, M)$.

An example of a semantically maximal solution for $\mathcal{P}_{1}$ is $\{$ hand-joint $\sqsubseteq$ joint, limb-joint $\sqsubseteq$ joint, autopod-joint $\sqsubseteq$ limb-joint $\}$. Semantically maximal solutions for $\mathcal{P}_{2}$ and $\mathcal{P}_{3}$ are \{hand-joint $\sqsubseteq$ joint, hinderlimb-joint $\sqsubseteq$ joint, forelimb-joint $\sqsubseteq$ joint, autopod-joint $\sqsubseteq$ limb-joint $\}$ and \{hand-joint $\sqsubseteq$ joint, hinderlimb-joint $\sqsubseteq$ joint-of-rib, forelimb-joint $\sqsubseteq$ joint-of-vertebralarch, autopod-joint $\sqsubseteq$ limb-joint $\}$, respectively. In the case of $\mathcal{P}_{4}$ a semantically maximal solution is $\{\mathrm{A} \sqsubseteq \mathrm{B} \mid \mathrm{A}, \mathrm{B} \in \mathrm{C}\}$.

Both the subset minimality and the semantic maximality are desirable. However, only with the semantic maximality we might obtain a solution with redundancy. Although subset minimality does not yield redundancy, there is no guarantee that the solution is the most informative. In practice, solutions with higher level of informativeness and no redundancy are most 
preferred as this means that a larger body of correct information is added to an ontology without the need for unnecessary validations. In the following we propose definitions on solutions by combining these criteria. There are diverse interpretations for the combination of subset minimality and semantic maximality, depending on what kind of priority we assign for the single preferences. A first interpretation implies a higher priority on subset minimality than the semantic maximality. In the second interpretation, we assign higher priority for semantic maximality than the subset minimality. In the third interpretation, the skyline-style interpretation, we treat both preferences equally and the chosen solution is such that there does not exist another solution which is preferable on both criteria.

Definition 6 (Combining with priority for subset minimality) $A$ solution $S$ to the GTAP $(T, C, O r, M)$ is said to be minmax optimal iff $S$ is subset minimal and there does not exist another subset minimal solution $S^{\prime}$ such that $S^{\prime}$ is more informative than $S$. The set of all minmax optimal solutions is denoted as $\mathcal{S}_{\min }^{\max }(T, C, O r, M)$.

Lemma $1 \mathcal{S}_{\min }^{\max }(T, C, O r, M) \subseteq \mathcal{S}_{\min }(T, C, O r, M)$

As an example, $\{$ limb-joint $\sqsubseteq$ joint $\}$ is a minmax optimal solution for $\mathcal{P}_{1}$, while $\{$ hinderlimb-joint $\sqsubseteq$ joint, forelimb-joint $\sqsubseteq$ joint $\}$ is a minmax optimal solution for $\mathcal{P}_{2}$. In the case of $\mathcal{P}_{3}$ we have a minmax solution \{hinderlimbjoint $\sqsubseteq$ joint-of-rib, forelimb-joint $\sqsubseteq$ joint-of-vertebral-arch $\}$.

In practice, minmax optimal solutions ensure fewer is-a relations to be added, thus avoiding redundancy. This is desirable if the domain expert would prefer to look at as small solutions as possible. The disadvantage is that there may be redundant relations that are correct which will not be derivable if not added.

Definition 7 (Combining with priority for semantic maximality) $A$ solution $S$ to the GTAP $(T, C, O r, M)$ is said to be maxmin optimal iff $S$ is semantically maximal and there does not exist another semantically maximal solution $S^{\prime}$ such that $S^{\prime}$ is a proper subset of $S$. The set of all maxmin optimal solutions is denoted as $\mathcal{S}_{\min }^{\max }(T, C, O r, M)$.

Clearly from the definition 7 the following relationship holds.

Lemma $2 \mathcal{S}_{\min }^{\max }(T, C, O r, M) \subseteq \mathcal{S}^{\max }(T, C, O r, M)$

As an example, \{limb-joint $\sqsubseteq$ joint, autopod-joint $\sqsubseteq$ limb-joint $\}$ is a maxmin optimal solution for $\mathcal{P}_{1}$. Examples of maxmin solutions for $\mathcal{P}_{2}$ and $\mathcal{P}_{3}$ are $\{$ hinderlimb-joint $\sqsubseteq$ joint, forelimb-joint $\sqsubseteq$ joint, autopod-joint $\sqsubseteq$ joint $\}$ and $\{$ hinderlimb-joint $\sqsubseteq$ joint-of-rib, forelimb-joint $\sqsubseteq$ joint-of-vertebralarch, autopod-joint $\sqsubseteq$ joint $\}$, respectively.

The advantage of the maxmin optimal semantics is that a maximal body of correct information is added to the ontology. If the domain expert would 
prefer to look at as informative solutions as possible without (set) redundancy, maxmin optimal solutions are more preferable than the minmax optimal solutions. This conclusion can even be strengthened from the efficiency point of view, as finding a maxmin optimal solution is more efficient than finding a minmax optimal one [96]. The disadvantage is that more relations need to be validated.

For the skyline interpretation, we consider the subset minimality and the semantic maximality as two dimensions for a solution $S$. $S$ is skyline optimal if it is not dominated by any other solution. A solution dominates another solution if it is as good or better in all dimensions and better in at least one dimension. Therefore regarding the above two dimensions we define that a solution $S$ dominates another solution $S^{\prime}$ if one of the following conditions is fulfilled:

1. $S \subsetneq S^{\prime}$ and $S$ is more informative than $S^{\prime}$, or

2. $S=S^{\prime}$ and $S$ is more informative than $S^{\prime}$, or

3. $S \subsetneq S^{\prime}$ and $S$ is equally informative as $S^{\prime}$.

It is easy to verify that condition 1 and 2 can never be fulfilled, due to the monotonicity property of the entailment. Therefore, a solution $S$ dominates another solution $S^{\prime}$ if and only if condition 3 is fulfilled. Accordingly, we have the definition for the skyline optimality as follows.

Definition 8 (Skyline optimal) A solution $S$ to the $G T A P(T, C, O r, M)$ is said to be skyline optimal iff there does not exist another solution $S^{\prime}$ such that $S^{\prime}$ is a proper subset of $S$ and $S^{\prime}$ is equally informative as $S$. The set of all skyline optimal solutions is denoted as $\mathcal{S}_{\min }^{\max }(T, C, O r, M)$.

Skyline optimal is a relaxed criterion. It requires subset minimality for some level of informativeness. It comprises all the subset minimal solutions - which in turn comprises all the minmax optimal solutions - and all the maxmin optimal solutions. This relationship can be easily verified.

Lemma $3 \mathcal{S}_{\min }(T, C, O r, M) \cup \mathcal{S}_{\min }^{\max }(T, C, O r, M) \subseteq \mathcal{S}_{\min }^{\max }(T, C, O r, M)$.

As an example, $M$ in Figure 3.1 is a skyline optimal solution for $\mathcal{P}_{1}$, $\mathcal{P}_{2}, \mathcal{P}_{3}$ and $\mathcal{P}_{4}$. All previous examples for subset minimal, minmax optimal and maxmin optimal solutions are also skyline optimal solutions. However, there are semantically maximal solutions that are not skyline optimal. For instance, $\{$ hinderlimb-joint $\sqsubseteq$ joint, forelimb-joint $\sqsubseteq$ joint, hand-joint $\sqsubseteq$ joint $\}$ is a semantically maximal solution for $\mathcal{P}_{2}$, but it is not skyline optimal as its subset $\{$ hinderlimb-joint $\sqsubseteq$ joint, forelimb-joint $\sqsubseteq$ joint $\}$ is equally informative. 


\subsection{Debugging in practice}

\subsubsection{General observations}

A system for repairing missing is-a structure in ontologies, takes as input the ontology $T$ and a set of is-a relations to repair $M$. $C$ is implicit and can be computed using $T$. Further, the system should be used by a domain expert who validates is-a relations $(O r)^{3}$. In general, however, when starting a debugging session, we do not know the properties of $T, M$ and $O r$. Further, Or represents the knowledge about is-a relations from the domain expert, but is normally not available beforehand, but only through interaction with the debugging system.

Table 3.1 provides us with some guidelines for the development and the use of debugging systems. First, it is clear that we would prefer an allknowing expert. The second best case for obtaining correct solutions is the partial-correct expert. As discussed in Section 3.1, this could be approximated by using multiple domain experts and a sceptical approach.

If there are wrong is-a relations in $M$, there will be no solution or solutions that are not correct. The repaired ontology will contain incorrect is-a relations. Therefore, the expert should validate $M$ at the beginning of the debugging session. Those is-a relations which are identified to be incorrect should be removed from $M .{ }^{4}$ Another advantage of the validation is that, after validation we have that $\forall \mathrm{m} \in M$ : $\operatorname{Or}(\mathrm{m})=$ true.

Further, as we do not know whether $T$ is correct according to the domain or not, it should be checked whether $T \cup M_{\text {validated }}$ is consistent. If not, then there are no solutions. Otherwise, we know that $M_{\text {validated }}$ is a solution. When we remove the redundancy from $M_{\text {validated }}$, then we also have a subset minimal solution. This solution could then be used as a basis for finding more informative solutions. The difficulty is in finding $\mathrm{S}=\left\{E_{i} \sqsubseteq F_{i}\right\}_{i=1}^{k}$ such that $\forall E_{i}, F_{i}: E_{i}, F_{i} \in C, \forall E_{i}, F_{i}: \operatorname{Or}\left(E_{i} \sqsubseteq F_{i}\right)=$ true and $T \cup S$ is consistent.

\subsubsection{Lessons for an existing system}

The system in [68] allows debugging the is-a structure of and mappings between taxonomies in a taxonomy network. The input to the system is an ontology network. In this discussion we focus on one of the ontologies in the network ( $T$ and thus also $C$ ). The debugging workflow consists of three phases: (1) detection (generation of $M),(2)$ validation of $M$ and (3) repair (solving the GTAP problem). The domain expert is involved in the validation of $M$ as well as in phase 3 for validation of possible solutions

\footnotetext{
${ }^{3}$ If there would be no expert, as shown in Table 3.1 , in the best case $M$ could be a correct solution, but there is no guarantee for solutions. We do not discuss this case further in this section.

${ }^{4}$ Depending on the detection method to generate $M$, the wrong is-a relations in $M$ may lead to other debugging opportunities for semantic defects (e.g., [54]).
} 
$(S)$. The domain expert can switch between the different phases at any time. The system was used in a real case for the Swedish National Food Agency [55] and in several experiments with ontologies from the Ontology Alignment Evaluation Initiative.

Although the system allows to switch between the different phases, in all experiments the authors started with validating $M$, which is as suggested by our analysis in Section 3.3.1. If $M$ contained wrong is-a relations, the authors used semantic debugging techniques to repair these. This allowed to remove incorrect is-a relations in $T$. When all the wrong is-a relations are repaired and removed from $M$, new $M_{\text {validated }}$ is obtained. If the domain expert validated $M$ in a correct way, then this represents a situation in the upper part of Table 3.1. The is-a relations in $M_{\text {validated }}$ are then repaired. When they are repaired using solutions that are more informative than $M_{\text {validated }}$, then new knowledge is added to the network and a new round of detection was started, possibly leading to the detection, validation and repair of new is-a relations.

Initially, $M_{\text {validated }}$ is added to the ontology. This means that the repairing process starts with a least informative solution. When removing redundancy from $M_{\text {validated }}$, it is also a subset minimal solution. Then, the system tries to generate more informative solutions. For this, the missing is-a relations are repaired one at the time. For each missing is-a relation $m_{i}$ a set of is-a relations $R_{i}$ is computed that guarantees that $T \cup\left\{r_{i}\right\}=m_{i}$ for each $r_{i} \in R_{i}$. Thus, for each missing is-a relation, at most one is-a relation is added to the ontology. By removing redundancy subset minimal solutions can be guaranteed. Further, for each missing is-a relation on its own semantically maximal solutions are generated with the extra conditions that only one is-a relation is used for repairing and no unnecessary equivalences $\left(\ll_{S H}\right.$ in [71]) are introduced in the ontology.

One immediate consequence of our analysis is that we should allow a domain expert to choose several elements of each $R_{i}$. This would be an easy extension to the system that would provide more informative solutions. Another consequence is that it would be advantageous to allow a domain expert to deal with a previously repaired is-a relation again, when new knowledge was added to the ontology. New more informative solutions may be found. Further, there should be a way for domain experts to add new is-a relations that do not occur within the repairing process.

An interesting observation during the debugging described in [55] was that the domain experts changed their mind about the correctness of some is-a relations after debugging some other is-a relations. This means that $O r$ may actually change during a session, and we may move upwards in Table 3.1 . 


\section{Chapter 4}

\section{Repairing missing is-a structure in $\mathcal{E} \mathcal{L}$ ontologies}

In the previous chapter we have formalized the problem of repairing missing is-a structure in ontologies as a generalized version of the TBox abduction problem. This chapter ${ }^{1}$ introduces our approach for repairing missing is-a structure in $\mathcal{E} \mathcal{L}$ ontologies. Our approach is based on the GTAP and we present an algorithm for solving the GTAP in ontologies represented in the $\mathcal{E} \mathcal{L}$ family of description logics. The $\mathcal{E} \mathcal{L}$ family of description logics is highly relevant for the representation of the lightweight ontologies. For instance, several of the major ontologies in the biomedical domain, e.g. SNOMED CT [6] and Gene Ontology [12], can be represented in $\mathcal{E} \mathcal{L}$ or small extensions thereof [13].

The proposed algorithm for solving the GTAP is iterative. The input is a set of detected validated missing is-a relations and the output from every iteration of the algorithm is a skyline optimal solution. The idea is that in every subsequent iteration we try to improve the solution from the previous iteration by finding a solution which introduces additional new knowledge.

We have developed a semi-automatic system for completing the is-a structure in $\mathcal{E} \mathcal{L}$ ontologies based on the algorithm. We evaluated the system in two experiments using biomedical ontologies. The results of the evaluation show the usefulness of the system as a large number of new is-a relations were added to ontologies which were not detected by the detection algorithm. In addition, the iterative approach proved to be beneficial given that in all experiments new relations were identified in subsequent iterations.

The chapter is organized as follows. First, we present an algorithm for solving the GTAP in $\mathcal{E} \mathcal{L}$ ontologies (Section 4.1). In Section 4.2 we extend the algorithm to deal with ontologies represented in $\mathcal{E} \mathcal{L}^{++}$. Next, we present an implemented system (Section 4.3) for completing the is-a structure in ontologies represented in the $\mathcal{E} \mathcal{L}$ family of description logics

\footnotetext{
${ }^{1}$ The chapter is based on $[32,33,96]$
} 
which is based on the algorithms. Finally, in Section 4.4 we provide an evaluation of the system using three ontologies from the biomedical domain and discuss lessons learned.

\subsection{Algorithm $-\mathcal{E} \mathcal{L}$}

In this section we present an algorithm for repairing missing is-a structure (solving GTAP $(T, C, O r, M))$ in ontologies that are represented in $\mathcal{E} \mathcal{L}$ and where the TBox is normalized as described in [13]. A normalized TBox $T$ contains only axioms of the forms $A_{1} \sqcap \ldots \sqcap A_{n} \sqsubseteq B, A \sqsubseteq \exists r . B$, and $\exists r . A \sqsubseteq B$, where $A, A_{1}, \ldots, A_{n}$ and $B$ are atomic concepts and $r$ is a role. Further, based on lessons learned in Section 3.3, we require that the missing is-a relations are validated before the repairing. We also note that $\mathcal{E} \mathcal{L}$ TBoxes are always consistent. Thus $\forall m \in M: \operatorname{Or}(m)=$ true, and $T \cup$ $M$ is consistent and therefore, $M$ is a solution. The algorithm in Algorithm 1 computes a skyline optimal solution for a $\operatorname{GTAP}(T, C, O r, M)$. As $M$ is a solution, the algorithm will always return a result. The result can be a subset minimal solution that is a subset of $M$ or a solution that is more informative than $M$.

The basic step in the algorithm (RepairSingleIsa) computes a solution for a GTAP with one missing is-a relation (i.e. GTAP $(T, C, O r,\{E \sqsubseteq F\})$ in the following way. First, superconcepts of E are collected in a Source set and subconcepts of $\mathrm{F}$ are collected in a Target set (lines 3 and 4). Source contains expressions of the forms $A$ and $\exists r$. A while Target contains expressions of the forms $A, A_{1} \sqcap \ldots \sqcap A_{n}$ and $\exists r . A$ where $A, A_{1}, \ldots, A_{n}$ are atomic concepts and $r$ is a role. Adding an is-a relation between an element in Source and an element in Target to the ontology would make $E \sqsubseteq F$ derivable (and thus this gives us logical solutions, but not necessarily solutions that are correct according to the domain). As we are interested in solutions containing is-a relations between atomic concepts, we check for every pair $(\mathrm{A}, \mathrm{B}) \in$ Source $\times$ Target whether $\mathrm{A}$ and $\mathrm{B}$ are atomic concepts and $\operatorname{Or}(A \sqsubseteq B)=$ true (i.e. correct according to the domain). If so, then this is a possible solution for GTAP $(T, C, O r,\{E \sqsubseteq F\})$. However, to conform to subset minimality and semantic maximality, if the current solution already contains is-a relations that would lead to the entailment of $A \sqsubseteq B$ then we do not use $A \sqsubseteq B$ (lines 8-9). Otherwise we use $A \sqsubseteq B$ and remove elements from the current solution that would be entailed if $A \sqsubseteq B$ is used (lines 10-12). Further, in the case where $\mathrm{A}$ is of the form $\exists r . N$ and $\mathrm{B}$ is of the form $\exists r . O$, then making $N \sqsubseteq O$ derivable would also make $A \sqsubseteq B$ derivable (lines 13-14). It is clear that for the result of RepairSingleIsa, i.e. Sol, the following holds: $T \cup S o l \models E \sqsubseteq F$ and $\forall s \in$ Sol $: \operatorname{Or}(s)=$ true. Together with the fact that $\mathcal{E} \mathcal{L}$ TBoxes are consistent, this leads to the fact that Sol is a solution of $\operatorname{GTAP}(T, C, O r,\{E \sqsubseteq F\})$.

In RepairMultipleIsa the algorithm collects for each missing is-a relation a solution from RepairSingleIsa and takes the union of these. There- 


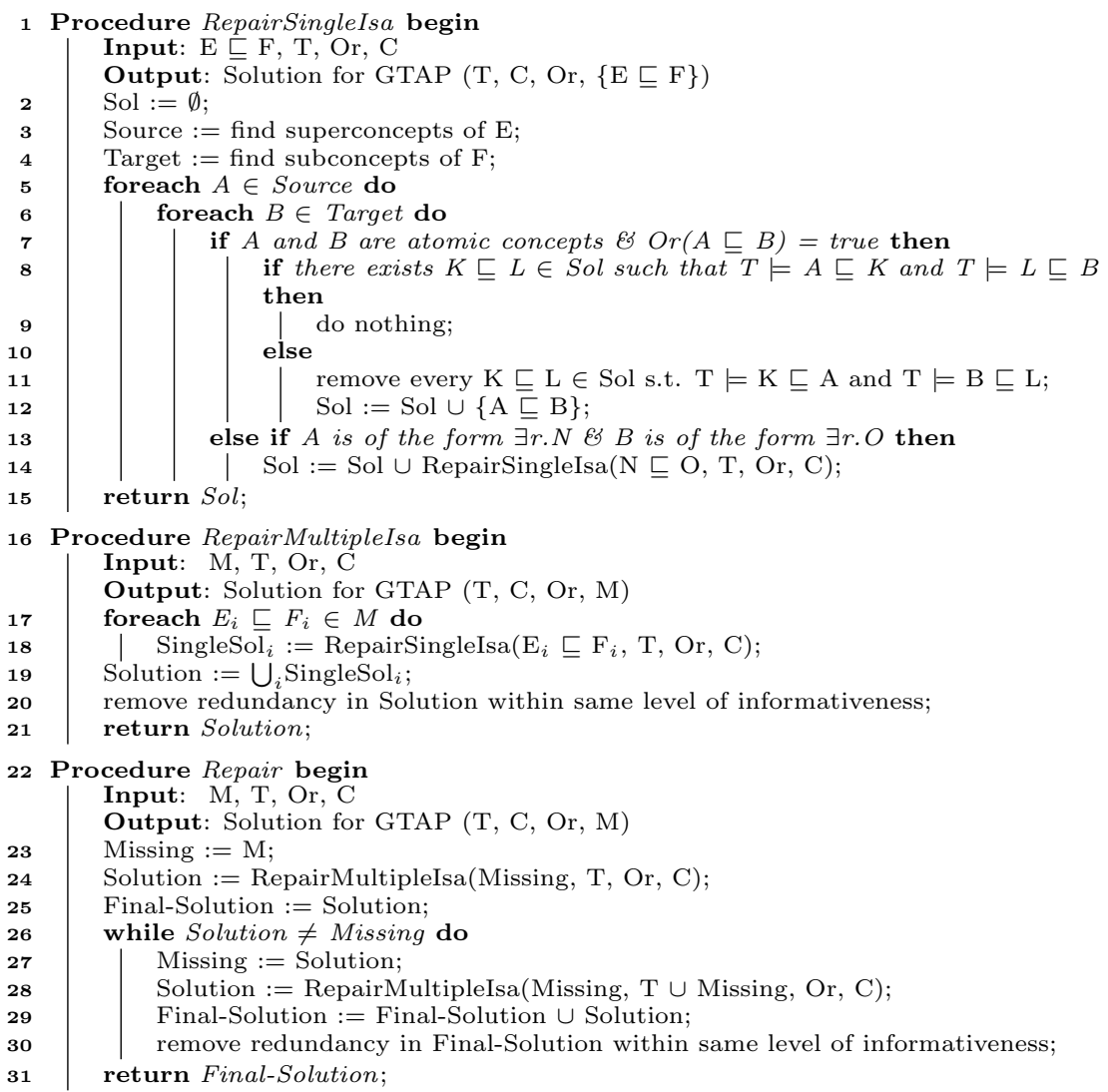

Algorithm 1: Algorithm for solving GTAP in $\mathcal{E} \mathcal{L}$ ontologies.

fore, the following holds for Solution in line 19: $T \cup$ Solution $\models M$ and $\forall s \in$ Solution : $\operatorname{Or}(s)=$ true. Together with the fact that $\mathcal{E} \mathcal{L}$ TBoxes are consistent, this leads to the fact that Solution is a solution of GTAP $(T, C, O r, M)$. Further, in line 20, we remove redundancy while keeping the same level of informativeness, and thus obtain a skyline optimal solution. (In the case where there are several ways to remove redundancy, one is chosen, as the extended ontologies will be equivalent in the sense that they entail the same statements.)

In Repair we try to improve the result from RepairMultipleIsa by trying to find a skyline optimal solution on a higher level of informativeness. Given that any element in the solution of RepairMultipleIsa that is not in $M$ can be considered as a new missing is-a relation (which was not detected earlier), we can try to find additional more informative ways of repairing by solving a new GTAP problem for these new missing is-a relations (and continue as long as new missing is-a relations are detected). As a (skyline optimal) solution for the new GTAP is also a (skyline optimal) solution of the original GTAP, 
the solution found in Repair is a skyline optimal solution for the original GTAP.

As an example run consider the GTAP in Figure 4.1. For a given ontology and set of missing is-a relations, the algorithm will first find solutions for repairing individual missing is-a relations using RepairSingleIsA. For the missing is-a relation Endocarditis $\sqsubseteq$ PathologicalPhenomenon the following is-a relations provide logical solutions for repairing the missing is-a relation: Endocarditis $\sqsubseteq$ PathologicalPhenomenon, Endocarditis $\sqsubseteq$ Fracture, Endocarditis $\sqsubseteq$ CardioVascularDisease, Carditis $\sqsubseteq$ PathologicalPhenomenon, Carditis $\sqsubseteq$ Fracture, Carditis $\sqsubseteq$ CardioVascularDisease as well as InflammationProcess $\sqsubseteq$ PathologicalProcess. As the first one is the missing is-a relation which was already validated, only the other six is-a relations are presented to the oracle for validation. Out of these six Endocarditis $\sqsubseteq$ Fracture and Carditis $\sqsubseteq$ Fracture are not correct according to the domain and are therefore not included in solutions. Further, relations Endocarditis $\sqsubseteq$ CardioVascularDisease, Endocarditis $\sqsubseteq$ PathologicalPhenomenon, Carditis $\sqsubseteq$ PathologicalPhenomenon are removed given it is possible to entail them from the ontology together with the remaining relations. Therefore, after validation, RepairSingleIsA returns \{InflammationProcess $\sqsubseteq$ PathologicalProcess, Carditis $\sqsubseteq$ CardioVascularDisease $\}$. The same process is repeated for the second missing is-a relation GranulomaProcess $\sqsubseteq$ NonNormalProcess. In this case the following is-a relations provide logical solutions for repairing the missing is-a relation: GranulomaProcess $\sqsubseteq$ NonNormalProcess and GranulomaProcess $\sqsubseteq$ PathologicalProcess. GranulomaProcess $\sqsubseteq$ NonNormalProcess is the missing is-a relation and was already validated as correct according to the domain. GranulomaProcess $\sqsubseteq$ PathologicalProcess is presented to the oracle and validated as correct according to the domain. As GranulomaProcess $\sqsubseteq$ NonNormalProcess can be entailed from the ontology together with GranulomaProcess $\sqsubseteq$ PathologicalProcess, RepairSingleIsA returns \{GranulomaProcess $\sqsubseteq$ PathologicalProcess\}. The solutions for the single is-a relations are then combined to form a solution for the set of missing is-a relations. In our case, there are no redundant relations and therefore RepairMultipleIsA returns \{InflammationProcess $\sqsubseteq$ PathologicalProcess, Carditis $\sqsubseteq$ CardioVascularDisease, GranulomaProcess $\sqsubseteq$ PathologicalProcess $\}$ which is a skyline optimal solution. In Repair the system tries to improve the acquired solution. This time the oracle is presented with a total of 13 relations for validation out of which only one is validated to be correct, i.e. GranulomaProcess $\sqsubseteq$ InflammationProcess. This is added to the solution. Given this new is-a relation, GranulomaProcess $\sqsubseteq$ PathologicalProces is removed from the solution as it can now be entailed from the ontology and GranulomaProcess $\sqsubseteq$ InflammationProcess. The new solution is \{InflammationProcess $\sqsubseteq$ PathologicalProcess, Carditis $\sqsubseteq$ CardioVascularDisease, GranulomaProcess $\sqsubseteq$ InflammationProcess $\}$. This is again a skyline optimal solution and it is more informative than the previous solution. As new missing is-a relations were detected, the repairing 


\begin{abstract}
$C=\{$ GranulomaProcess, CardioVascularDisease, PathologicalPhenomenon, Fracture, Endocarditis, Carditis, InflammationProcess, PathologicalProcess, NonNormalProcess $\}$

$T=\{$ CardioVascularDisease $\sqsubseteq$ PathologicalPhenomenon, Fracture $\sqsubseteq$ PathologicalPhenomenon, $\exists$ hasAssociatedProcess.PathologicalProcess $\sqsubseteq$ PathologicalPhenomenon, Endocarditis $\sqsubset$ Carditis, Endocarditis $\sqsubset \exists$ hasAssociatedProcess.InflammationProcess, PathologicalProcess $\sqsubseteq$ NonNormalProcess $\}$

$M=\{$ Endocarditis $\sqsubseteq$ PathologicalPhenomenon, GranulomaProcess $\sqsubseteq$ NonNormalProcess $\}$

The following is-a relations are correct according to the domain, i.e., Or returns true for: GranulomaProcess $\sqsubset$ InflammationProcess, GranulomaProcess $\sqsubset$ PathologicalProcess, GranulomaProcess $\sqsubseteq$ NonNormalProcess, CardioVascularDisease $\sqsubseteq$ PathologicalPhenomenon, Fracture $\sqsubseteq$ PathologicalPhenomenon, Endocarditis $\sqsubseteq$ PathologicalPhenomenon, Endocarditis $\sqsubseteq$ Carditis, Endocarditis $\sqsubseteq$ CardioVascularDisease, Carditis $\sqsubseteq$ PathologicalPhenomenon, Carditis $\sqsubseteq$ CardioVascularDisease, InflammationProcess $\sqsubseteq \overline{\text { Pathological- }}$ Process, InflammationProcess $\sqsubseteq$ NonNormalProcess, PathologicalProcess $\sqsubseteq$ NonNormalProcess.

Let $\mathcal{P}=\operatorname{GTAP}(T, C, O r, M)$
\end{abstract}

Figure 4.1: Small $\mathcal{E} \mathcal{L}$ example.

is run for the third time. However, in this run the solution is not improved and thus the algorithm outputs the final result. We note that in this example we found a skyline optimal solution that is also semantically maximal. In general, however, it is not possible to know whether the solution is semantically maximal without checking every possible is-a relation between atomic concepts in the ontology.

\title{
$4.2 \quad$ Algorithm $-\mathcal{E} \mathcal{L}++$
}

As described in Section 2.2.1, unlike an $\mathcal{E} \mathcal{L}$ TBox which only contains a finite set of GCIs, an $\mathcal{E} \mathcal{L}^{++}$TBox can also contain role inclusions. On top of this, concepts descriptions can be formed using nominals as well as bottom concept $(\perp)$. Given these differences, a normalized an $\mathcal{E L}^{++}$TBox contains axioms of the forms $A_{1} \sqcap \ldots \sqcap A_{n} \sqsubseteq B, A_{1} \sqsubseteq \exists r . A_{2}$, and $\exists r . A_{1} \sqsubseteq$ $B$, as well as role inclusions of the forms $r \sqsubseteq s$ and $r_{1} \circ r_{2} \sqsubseteq s$ where $A_{1}$, $\ldots, A_{n}$ are atomic concepts, $B$ is either an atomic concept or the bottom concept and $r, r_{1}$ and $r_{2}$ are roles [13]. We note that $\mathcal{E} \mathcal{L}^{++}$TBoxes can be inconsistent. Thus $M$ is a solution iff $T \cup M$ is consistent. Therefore, in addition to the missing is-a relations being validated before the repairing i.e. $\forall m \in M: O r(m)=$ true, we require that $T \cup M$ is consistent.

The extended algorithm which deals with ontologies represented in $\mathcal{E L}^{++}$ is given in Algorithm 2. The extended algorithm works essentially in the same way as the $\mathcal{E} \mathcal{L}$ algorithm (Algorithm 1) except for two extensions which deal with additional expressivity of $\mathcal{E} \mathcal{L}^{++}$. First, given that $\mathcal{E L}^{++}$allows role inclusion, in the case where $\mathrm{A}$ is of the form $\exists r . N$ and $\mathrm{B}$ is of the form $\exists s . O$ and $\mathrm{r} \sqsubseteq \mathrm{s}$ holds, then making $N \sqsubseteq O$ derivable would also make $A \sqsubseteq B$ derivable. Similarly, if the TBox contains a role inclusion $r \circ r_{1} \sqsubseteq s$ 
$C=\{$ GranulomaProcess, CardioVascularDisease, PathologicalPhenomenon, Fracture, Endocarditis, Carditis, InflammationProcess, PathologicalProcess, NonNormalProcess, Wound, BurningProcess, SoftTissueTraumaProcess, TraumaticProcess

$T=\{$ CardioVascularDisease $\sqsubseteq$ PathologicalPhenomenon, Fracture $\sqsubseteq$ PathologicalPhenomenon, ᄏisImmediateConsequence.PathologicalProcess $\sqsubset$ PathologicalPhenomenon, Endocarditis $\sqsubseteq$ Carditis, Endocarditis $\sqsubseteq \exists$ isImmediateConsequence.InflammationProcess, PathologicalProcess $\sqsubseteq$ NonNormalProcess, hasAssociatedProcess $\sqsubset$ isImmediateConsequence, Wound $\sqsubseteq \exists$ has AssociatedProcess.SoftTissueTraumaProcess $\bar{\xi}$

$M=\{$ Endocarditis $\sqsubseteq$ PathologicalPhenomenon, GranulomaProcess $\sqsubseteq$ NonNormalProcess, Wound $\sqsubseteq$ PathologicalPhenomenon, BurningProcess $\sqsubseteq$ SoftTissueTraumaProcess, BurningProcess $\sqsubseteq$ TraumaticProcess $\}$

The following is-a relations are correct according to the domain, i.e $O r$ returns true for: GranulomaProcess $\sqsubseteq$ InflammationProcess, GranulomaProcess $\sqsubseteq$ PathologicalProcess, GranulomaProcess $\sqsubseteq$ NonNormalProcess, CardioVascularDisease $\sqsubseteq$ PathologicalPhenomenon, Fracture $\sqsubset$ PathologicalPhenomenon, Endocarditis $\sqsubset$ PathologicalPhenomenon, Endocarditis $\sqsubseteq$ Carditis, Endocarditis $\sqsubseteq$ CardioVascularDisease, Carditis $\sqsubseteq$ PathologicalPhenomenon, Carditis $\sqsubseteq$ CardioVascularDisease, InflammationProcess $\sqsubseteq$ PathologicalProcess, InflammationProcess $\sqsubseteq$ NonNormalProcess, PathologicalProcess $\sqsubseteq$ NonNormalProcess, Wound $\sqsubseteq$ PathologicalPhenomenon, TraumaticProcess $\sqsubseteq$ NonNormalProcess, TraumaticProcess $\sqsubseteq$ PathologicalProcess, SoftTissueTraumaProcess $\sqsubseteq$ TraumaticProcess, SoftTissueTraumaProcess $\sqsubseteq$ NonNormalProcess, SoftTissueTraumaProcess $\sqsubseteq$ PathologicalProcess, BurningProcess $\sqsubseteq$ NonNormalProcess, BurningProcess $\sqsubseteq$ PathologicalProcess, BurningProcess $\sqsubseteq$ SoftTissueTraumaProcess, BurningProcess $\sqsubseteq$ TraumaticProcess.

Let $\mathcal{P}=\operatorname{GTAP}(T, C, O r, M)$.

Figure 4.2: Small $\mathcal{E} \mathcal{L}^{++}$example.

and axiom $N \sqsubseteq \exists r_{1} . P$ then adding $P \sqsubseteq O$ would also make $A \sqsubseteq B$ derivable. These types of solutions are found in procedure FindExistsSolutions. Second, given that $\mathcal{E} \mathcal{L}^{++}$TBoxes can contain inconsistencies the extended algorithm contains a check for consistency of solutions for individual missing is-a relations (line 7) as well as an additional check in the RepairMultipleIsa procedure (line 23). In the latter case, if the union of the solution and the TBox is inconsistent then the algorithm will return the solution from the previous iteration and therefore terminate the algorithm. This makes the extended algorithm sound and it guarantees a solution iff $\mathrm{T} \cup \mathrm{M}$ is consistent ( $\mathrm{M}$ is a solution).

Same as with the $\mathcal{E} \mathcal{L}$ algorithm, the extended algorithm computes a skyline optimal solution for a $\operatorname{GTAP}(T, C, O r, M)$.

As an example let us consider the GTAP in Figure 4.2 which is an extension of the GTAP in Figure 4.1. First step of the algorithm is to find solutions to GTAPs for individual missing is-a relations.

In the case of missing is-a relation Endocarditis $\sqsubseteq$ PathologicalPhenomenon the algorithm find the same 7 logical solutions as in the $\mathcal{E} \mathcal{L}$ example out of which Endocarditis $\sqsubseteq$ Fracture and Carditis $\sqsubseteq$ Fracture are validated to be not correct according to the domain. The non-redundant solution for missing is-a relation Endocarditis $\sqsubseteq$ PathologicalPhenomenon is $\{$ InflammationProcess $\sqsubseteq$ PathologicalProcess, Carditis $\sqsubseteq$ CardioVascularDisease $\}$. 


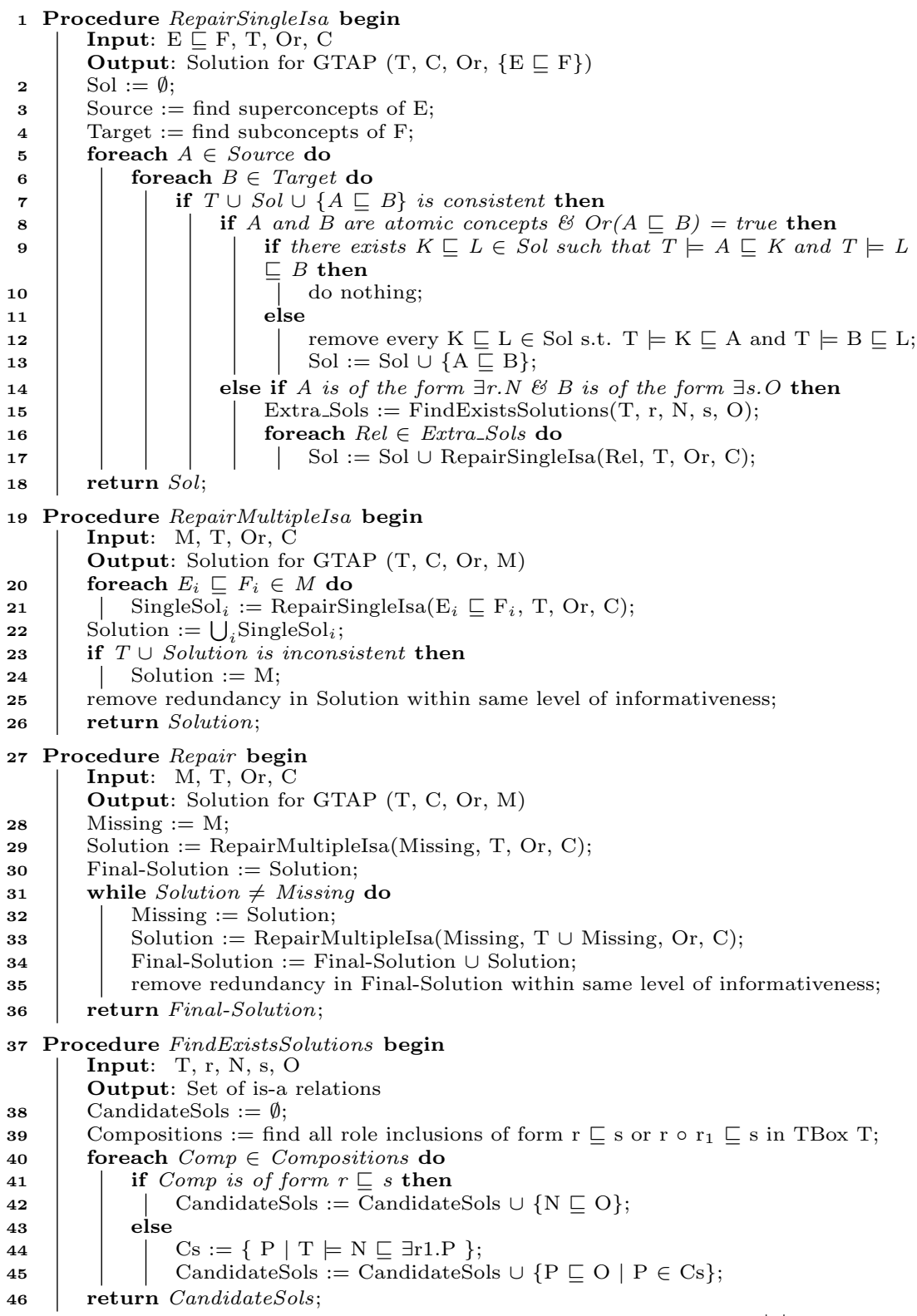

Algorithm 2: Algorithm for solving GTAP in $\mathcal{E L}^{++}$.

The process continues with the second missing is-a relation GranulomaProcess $\sqsubseteq$ NonNormalProcess. The solution for this missing is-a relation is the same as in the $\mathcal{E} \mathcal{L}$ example, i.e., RepairSingleIs $A$ returns $\{$ GranulomaProcess $\sqsubseteq$ PathologicalProcess $\}$ given that is-a relation GranulomaProcess $\sqsubseteq$ Non- 
NormalProcess is redundant.

For the missing is-a relation Wound $\sqsubseteq$ PathologicalPhenomenon relations Wound $\sqsubseteq$ PathologicalPhenomenon, SoftTissueTraumaProcess $\sqsubseteq$ PathologicalProcess, Wound $\sqsubseteq$ Fracture, Wound $\sqsubseteq$ CardioVascularDisease provide logical solutions for repairing the missing is-a relation. Out of these, only Wound $\sqsubseteq$ PathologicalPhenomenon and SoftTissueTraumaProcess $\sqsubseteq$ PathologicalProcess are correct according to the oracle, and RepairSingleIsA therefore returns $\{$ Wound $\sqsubseteq$ PathologicalPhenomenon, SoftTissueTraumaProcess $\sqsubseteq$ PathologicalProcess $\}$.

For the remaining missing is-a relations BurningProcess $\sqsubseteq$ SoftTissueTraumaProcess and BurningProcess $\sqsubseteq$ TraumaticProcess the procedure RepairSingleIsA returns \{BurningProcess $\sqsubseteq$ SoftTissueTraumaProcess $\}$ and \{BurningProcess $\sqsubseteq$ TraumaticProcess $\}$ respectively. The solutions for the single is-a relations are then combined to form a solution for the set of missing is-a relations. In our case, Wound $\sqsubseteq$ PathologicalPhenomenon is redundant and therefore RepairMultipleIs $A$ returns InflammationProcess $\sqsubseteq$ PathologicalProcess, Carditis $\sqsubseteq$ CardioVascularDisease, GranulomaProcess $\sqsubseteq$ PathologicalProcess, BurningProcess $\sqsubseteq$ TraumaticProcess, BurningProcess $\sqsubseteq$ SoftTissueTraumaProcess, SoftTissueTraumaProcess $\sqsubseteq$ PathologicalProcess $\}$. This is a skyline optimal solution and in Repair the system tries to improve the acquired solution. This time the oracle is presented with a total of 29 relations for validation out of which only two are validated to be correct, i.e. GranulomaProcess $\sqsubseteq$ InflammationProcess and SoftTissueTraumaProcess $\sqsubseteq$ TraumaticProcess. These are added to the solution. Given these new is-a relations, GranulomaProcess $\sqsubseteq$ PathologicalProces and SoftTissueTraumaProcess $\sqsubseteq$ TraumaticProcess are removed from the solution as they are redundant. The new solution is \{InflammationProcess $\sqsubseteq$ PathologicalProcess, Carditis $\sqsubseteq$ CardioVascularDisease, GranulomaProcess $\sqsubseteq$ InflammationProcess, SoftTissueTraumaProcess $\sqsubseteq$ TraumaticProcess, BurningProcess $\sqsubseteq$ SoftTissueTraumaProcess, SoftTissueTraumaProcess $\sqsubseteq$ PathologicalProcess $\}$. This is again a skyline optimal solution and it is more informative than the previous solution. Given that new missing is-a relations were detected, we run the repairing again. In this iteration 4 new relations required validation and only relation TraumaticProcess $\sqsubseteq$ PathologicalProcess is validated as correct according to the domain. The new solution is \{nflammationProcess $\sqsubseteq$ PathologicalProcess, Carditis $\sqsubseteq$ CardioVascularDisease, GranulomaProcess $\sqsubseteq$ InflammationProcess, SoftTissueTraumaProcess $\sqsubseteq$ TraumaticProcess, BurningProcess $\sqsubseteq$ SoftTissueTraumaProcess, TraumaticProcess $\sqsubseteq$ PathologicalProcess $\}$. The relation SoftTissueTraumaProcess $\sqsubseteq$ PathologicalProcess was removed from the solution as it is redundant.

The algorithm is run again and in this iteration no new is-a relations were validated to be correct so the solution from the previous iteration is returned as the final solution. 


\subsection{System}

We have implemented a system for completing the missing is-a structure in $\mathcal{E} \mathcal{L}^{++}$ontologies based on the algorithm in Algorithm 2. The input to the system is a an ontology and a set of validated missing is-a relations. The output is a solution to GTAP (called a repairing action). The system was implemented in Java and uses the ELK reasoner (version 0.4.1) [61] to detect implicit entailments in the ontology. The system is semi-automatic and requires interaction with a user which is a domain expert serving as an oracle and who decides whether an is-a relation is correct according to the domain.

Once the ontology and the set of missing is-a relations are loaded, the user starts the debugging process by pressing the button Generate Repairing Actions. The system then removes redundant is-a relations and the non-redundant missing is-a relations are shown in a drop-down list allowing the user to switch between missing is-a relations. Additional relations acquired from the FindExistsSolutions procedure in the algorithm (Algorithm 2) are also included in the drop-down list. It is also possible to scroll between relations using the arrow buttons in the bottom part of the screen.

After selecting an is-a relation from the list, the user is presented with the Source and the Target set for that is-a relation. The user then needs to choose relations which are correct according to the domain for that is-a relation. Missing is-a relations are automatically validated to be correct according to the domain while the relations that were acquired using $\exists$ (FindExistsSolutions procedure) in the algorithm have to be explicitly validated by the user. The system also implements an optimization, i.e. for a missing is-a relation $\mathrm{A} \sqsubseteq \mathrm{B}$ we remove superconcepts of $\mathrm{B}$ from Source, and the subconcepts of A from Target. Because of this, it is not possible to introduce non-validated equivalence relations where in the original ontology there are only is-a relations. For example, for a missing is-a relation $\mathrm{A} \sqsubseteq \mathrm{B}$ let us assume that both $\mathrm{A}$ and $\mathrm{B}$ have a superconcept $\mathrm{C}$. Adding the relation $\mathrm{C} \sqsubseteq \mathrm{B}$ would repair the missing is-a relation as well as change the existing relation $\mathrm{B} \sqsubseteq \mathrm{C}$ in the ontology into an equivalence relation which was not validated by the domain expert. This optimization also reduces the size of Source and Target sets.

In Figure 4.3(a) the user is presented with the Source and the Target set for the missing is-a relation Endocarditis $\sqsubseteq$ PathologicalPhenomenon (concepts in the missing is-a relation are marked in red). In this case the user has selected $\{$ Carditis $\sqsubseteq$ CardioVascularDisease $\}$ as a repairing action for the missing is-a relation (concepts marked in purple) and needs to confirm this by clicking the Validate button.

The user also has the option to check which relations have been validated so far and which relations can be validated, by clicking the Validate Is-a Relations button. In the pop-up window that appears the user can validate new relations, remove validations from already validated relations 


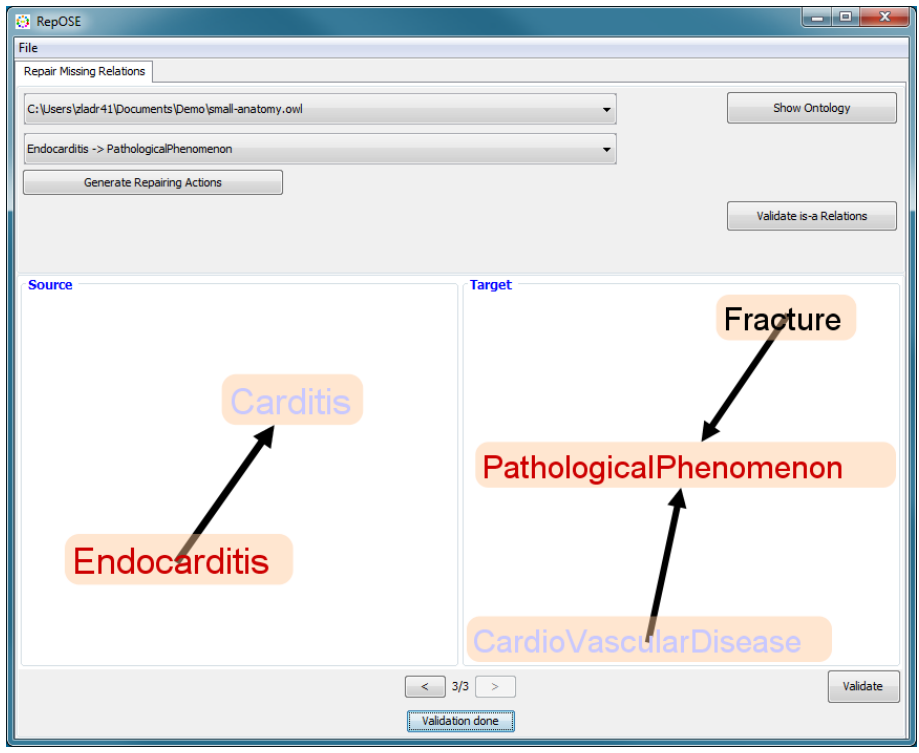

(a) Repairing using Source and Target sets.

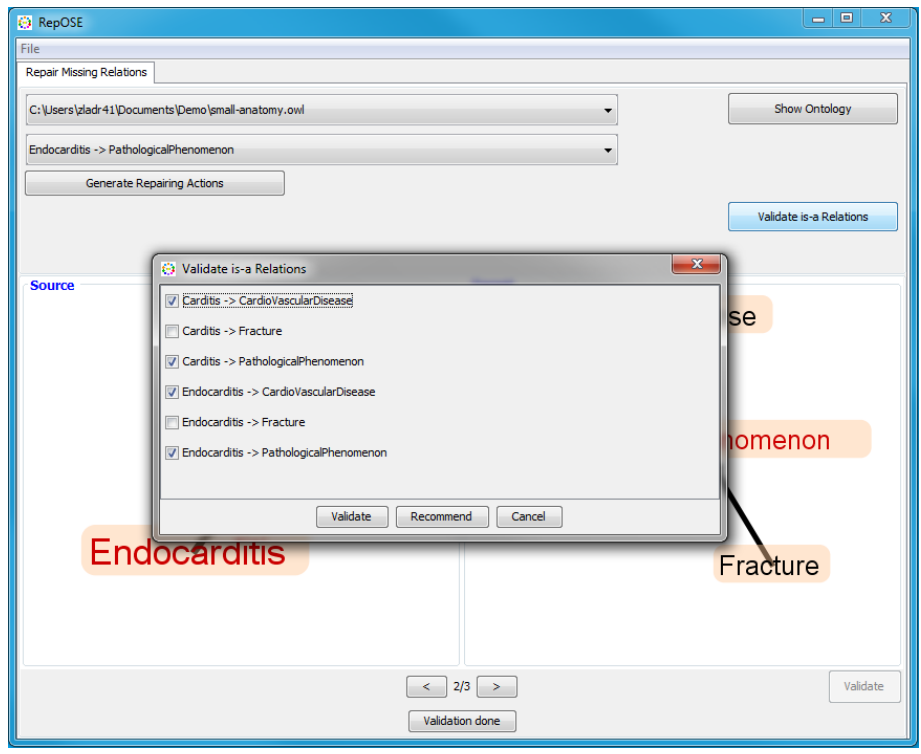

(b) Validating is-a relations.

Figure 4.3: System for repairing $\mathcal{E} \mathcal{L}$ ontologies - screenshots.

as well as ask for a recommendation by clicking the Recommend button (Figure 4.3(b)). Recommendations are acquired by querying external sources (currently, WordNet, UMLS Methathesaurus [8] and Uberon [7]). More 
specifically, each is-a relation which can be formed by taking a concept from Source and a concept from Target is checked in the external source if it exists. $^{2}$

The validation phase is ended by clicking on the Validation Done button. The system then calculates the consequences of the chosen repairing actions and presents the user with a new set of is-a relations that need to be repaired. The validation phase and consequent computations represent one iteration of the Repair procedure in Algorithm 2. If the repairing did not change between two iterations the system outputs the repairing.

At any point the user can save validated relations from the File menu which makes it possible to do debugging across multiple sessions.

\subsection{Experiments}

We have run several experiments on an Intel Core i7-2620M Processor at 3.07 GHz with 4 GB RAM under Windows 7 Professional and Java 1.7 Runtime Environment. In all experiments the validation phase took the most time while the computations between iterations took less than 10 seconds.

The results are summarized in Tables 4.1, 4.3 and 4.5. The 'It' columns represent the different iterations of Repair in Algorithm 2. The 'Missing' rows give the number of missing is-a relations in each iteration. Such a missing is-a relation can be repaired by adding itself ('Repaired by itself'), by adding other is-a relations that were not derivable in the ontology and thus represent new knowledge added to the ontology ('Repaired using new knowledge'). The 'New relations' row shows how many new is-a relations were added to the ontology. When such relations were found using $\exists$, then the number of such relations is shown in parentheses. We note that for iteration $i+1$ the number of missing is-a relations is the number of new relations from iteration $i$ plus the number of missing is-a relations repaired by themselves from iteration $i$ if there are no redundant relations. We also note that in the last iteration all missing is-a relations from that iteration are always repaired by themselves and these represent the final repairing action.

We experimented with repairing an ontology for which we randomly removed is-a relations and then repaired the ontology. Further, we debugged the two ontologies from the Anatomy track at the 2013 Ontology Alignment Evaluation Initiative.

\subsubsection{Experiment 1 - OAEI Anatomy}

We debugged the two ontologies from the Anatomy track at the 2013 Ontology Alignment Evaluation Initiative, i.e. Adult Mouse Anatomy ontology (AMA) containing 2744 concepts and a fragment of NCI human anatomy

\footnotetext{
${ }^{2}$ An optimized version of this approach is shown in [71].
} 


\begin{tabular}{|l|c|c|c|}
\hline & It1 & It2 & It3 \\
\hline Missing & 94 & 101 & 101 \\
Repaired by itself & 57 & 98 & 101 \\
Repaired using new knowledge & 37 & 3 & 0 \\
New relations & 44 & 3 & 0 \\
\hline
\end{tabular}

Table 4.1: Results for debugging AMA - Adult Mouse Anatomy ontology.

\begin{tabular}{|l|l|l|l|l|l|}
\hline & 1 & $2-10$ & $11-20$ & $21-30$ & $31-40$ \\
\hline AMA - Source & $56 / 66 / 67$ & $38 / 35 / 34$ & $0 / 0 / 0$ & $0 / 0 / 0$ & $0 / 0 / 0$ \\
AMA - Target & $34 / 12 / 12$ & $12 / 43 / 43$ & $10 / 20 / 21$ & $0 / 1 / 1$ & $3 / 3 / 3$ \\
\hline & $41-50$ & $51-100$ & $101-200$ & $201-300$ & $301-400$ \\
\hline AMA - Source & $0 / 0 / 0$ & $0 / 0 / 0$ & $0 / 0 / 0$ & $0 / 0 / 0$ & $0 / 0 / 0$ \\
AMA - Target & $6 / 6 / 6$ & $4 / 3 / 3$ & $21 / 12 / 11$ & $0 / 1 / 1$ & $4 / 0 / 0$ \\
\hline
\end{tabular}

Table 4.2: Source and Target set sizes for debugging AMA - Adult Mouse Anatomy ontology. The $\mathrm{x} / \mathrm{y} / \mathrm{z}$ values represent the sizes for iteration 1,2 and 3 , respectively.

ontology (NCI-A) containing 3304 concepts. The input missing is-a relations for these two experiments were a set of 94 and 58 missing is-a relations, respectively, for AMA and NCI-A. These missing is-a relations were obtained by using a logic-based approach using an alignment between AMA and NCIA [70] to generate candidate missing is-a relations which were then validated by a domain expert to obtain actual missing is-a relations.

\section{AMA - Adult Mouse Anatomy}

The results for debugging AMA are given in Table 4.1. Three iterations were required to reach the final solution. Out of 94 initial missing is-a relations 37 were repaired by repairing actions which add new knowledge to the ontology while 57 were repaired using only the missing is-a relation itself. In total 44 new and non-redundant relations were added to the ontology in the first iteration. Out of 37 relations which were repaired by adding new relations, 22 had more than 1 non-redundant relation in the repairing action. For example, the missing is-a relation wrist joint $\sqsubseteq$ joint is repaired by a repairing action $\{$ limb joint $\sqsubseteq$ joint, wrist joint $\sqsubseteq$ synovial joint $\}$.

The set of missing is-a relations in the second iteration contains 101 relations, i.e. 57 relations which were repaired by adding the missing is-a relation itself and 44 newly added relations. In this iteration, 3 is-a relations were repaired by adding new knowledge to the ontology. All 3 of these is-a relations are is-a relations which were added in the previous iteration. For example, is-a relation wrist joint $\sqsubseteq$ synovial joint is repaired by a repairing action $\{$ wrist joint $\sqsubseteq$ hand joint \} which is possible given that the is-a rela- 


\begin{tabular}{|l|c|c|c|}
\hline & It1 & It2 & It3 \\
\hline Missing & 58 & 55 & 54 \\
Repaired by itself & 49 & 50 & 54 \\
Repaired using new knowledge & 9 & 5 & 0 \\
New relations & 6 & 4 & 0 \\
\hline
\end{tabular}

Table 4.3: Results of debugging NCI-A - Human Anatomy ontology

\begin{tabular}{|l|l|l|l|l|l|}
\hline & 1 & $2-10$ & $11-20$ & $21-30$ & $31-40$ \\
\hline NCI-A - Source & $17 / 23 / 22$ & $41 / 32 / 32$ & $0 / 0 / 0$ & $0 / 0 / 0$ & $0 / 0 / 0$ \\
NCI-A - Target & $35 / 7 / 9$ & $12 / 35 / 32$ & $3 / 5 / 5$ & $0 / 1 / 1$ & $0 / 0 / 0$ \\
\hline & $41-50$ & $51-100$ & $101-200$ & $201-300$ & $301-400$ \\
\hline NCI-A - Source & $0 / 0 / 0$ & $0 / 0 / 0$ & $0 / 0 / 0$ & $0 / 0 / 0$ & $0 / 0 / 0$ \\
NCI-A - Target & $0 / 0 / 0$ & $4 / 3 / 3$ & $1 / 2 / 2$ & $1 / 1 / 1$ & $2 / 1 / 1$ \\
\hline
\end{tabular}

Table 4.4: Source and Target set sizes for debugging NCI-A - Human Anatomy ontology. The $\mathrm{x} / \mathrm{y} / \mathrm{z}$ values represent the sizes for iteration 1 , 2 and 3 , respectively.

tion metacarpo-phalangeal joint $\sqsubseteq$ joint from the initial set of missing is-a relations was repaired by a repairing action $\{$ hand joint $\sqsubseteq$ synovial joint, limb joint $\sqsubseteq$ joint $\}$ in the first iteration. Finally, the set of missing is-a relations containing 101 is-a relations in the third iteration is also the solution for the initial set of missing is-a relations given that no new relations were added in the third iteration.

The sizes of Source and Target sets for different iterations of the experiment are given in Table 4.2. A large portion of all Source and Target sets contained less then 10 concepts which implies that the domain correct relations are not to difficult to visualize. In cases with large Source and Target sets the current visualization became cluttered and the validation was more easily done in the table form which is acquired by pressing Validate Is-a Relations button.

\section{NCI - Human Anatomy}

The initial set of missing is-a relations contained 58 relations for the NCI-A ontology. Out of these 58 relations, in the first iteration, 9 were repaired by adding relations which introduce new knowledge to the ontology. In total 6 new is-a relations were added in this iteration.

In the second iteration, 5 out of 55 is-a relations were repaired by adding new relations while repairing actions for the 50 other is-a relations were unchanged. All 5 is-a relations which were repaired by adding new relations to the ontology are is-a relations which were repaired by repairing actions containing only the missing is-a relation from the first iteration. This ex- 


\begin{tabular}{|l|c|c|c|c|}
\hline & It1 & It2 & It3 & It4 \\
\hline Missing & 47 & 41 & 42 & 41 \\
Repaired by itself & 19 & 31 & 38 & 41 \\
Repaired using new knowledge & 28 & 10 & 4 & 0 \\
New relations & $26(3)$ & 11 & $3(1)$ & 0 \\
\hline
\end{tabular}

Table 4.5: Results for debugging the BioTop ontology.

\begin{tabular}{|l|l|l|l|l|l|}
\hline & 1 & $2-10$ & $11-20$ & $21-30$ & $31-40$ \\
\hline BioTop - Source & $26 / 44 / 48 / 53$ & $24 / 18 / 15 / 0$ & $0 / 0 / 0 / 0$ & $0 / 0 / 0 / 0$ & $0 / 0 / 0 / 0$ \\
BioTop - Target & $9 / 15 / 17 / 13$ & $28 / 22 / 23 / 19$ & $5 / 6 / 6 / 6$ & $1 / 10 / 8 / 7$ & $0 / 2 / 2 / 2$ \\
\hline & $41-50$ & $51-100$ & $101-200$ & $201-300$ & $301-400$ \\
\hline BioTop - Source & $0 / 0 / 0 / 0$ & $0 / 0 / 0 / 0$ & $0 / 0 / 0 / 0$ & $0 / 0 / 0 / 0$ & $0 / 0 / 0 / 0$ \\
BioTop - Target & $0 / 1 / 2 / 0$ & $7 / 1 / 2 / 4$ & $0 / 5 / 2 / 1$ & $0 / 0 / 1 / 1$ & $0 / 0 / 0 / 0$ \\
\hline
\end{tabular}

Table 4.6: Source and Target set sizes for debugging the BioTop ontology. The $\mathrm{x} / \mathrm{y} / \mathrm{z} / \mathrm{u}$ values represent the sizes for iteration $1,2,3$ and 4 , respectively.

emplifies why it is beneficial to consider already repaired is-a relations in subsequent iterations as Source and Target sets for some missing is-a relations can change and more informative solutions might be identified. The input to the third iteration is a set of 54 is-a relations and given that no changes were made, these relations are the final solution.

The sizes of Source and Target sets for different iterations of the experiment are given in Table 4.4. The same comments about the sizes of Source and Target sets as in the AMA - Adult Mouse Anatomy experiment hold.

\subsubsection{Experiment 2 - BioTop}

In this experiment we used the BioTop ontology from the 2013 OWL Reasoner Evaluation Workshop dataset containing 280 concepts and 42 object properties. For the set of missing is-a relations we randomly selected 47 is-a relations. Then the ontology was modified by removing is-a relations which would make the selected is-a relations derivable. The unmodified ontology was used as domain knowledge in the experiment. The results for debugging BioTop ontology are presented in Figure 4.5.

The debugging process took 4 iterations. In the first iteration 28 relations were repaired by adding new relations. In total 26 new relations were added in the first iteration out of which 3 using axioms containing $\exists$ expressions. For example, for missing is-a relation GreatApe $\sqsubseteq$ Primate we have a repairing action $\{$ FamilyHominidaeQuality $\sqsubseteq$ OrderPrimatesQuality $\}$ given that the ontology contains axioms GreatApe $\sqsubseteq \exists$ hasInherence.FamilyHominidaeQuality and $\exists$ hasInherence.OrderPrimatesQuality $\sqsubseteq$ Primate. 
The input to the second iteration contained 41 non-redundant is-a relations ( 4 redundant is-a relations were removed from the solution in iteration 1). In total 10 is-a relations were repaired by adding new is-a relations. Out of these 10 repaired is-a relations, 4 are relations from the initial set of missing is-a relations while the other 6 are relations which were added in the first iteration. For example, is-a relation Atom $\sqsubseteq$ Entity from the initial set of missing relations can be repaired with $\{$ Atom $\sqsubseteq$ MaterialEntity $\}$ given that MaterialEntity $\sqsubseteq$ Entity was added in the previous iteration.

In the third iteration, the input contained 42 is-a relations. In total 4 is-a relations ( 3 from the initial set of missing is-a relations and 1 from the first iteration) were repaired by adding 3 new relations. Out of the 3 new relations 1 is acquired using axioms containing $\exists$ expressions. Finally, in the fourth iteration no new relations were added and the system outputs the solution.

The sizes of Source and Target sets for different iterations of the experiment are given in Table 4.6. The same comments about the sizes of Source and Target sets as in the previous experiments hold.

\subsubsection{Lessons Learned}

The experiments have shown the usefulness of our approach. In each of the cases, whether missing is-a relations were identified, or whether we investigated existing is-a relations, our approach identified new information to be added to the ontologies.

The experiments have also shown that the iterative approach to repairing missing is-a relations is beneficial as in all our experiments additional relations were added to the ontology in subsequent iterations. Running the system on already repaired is-a relations gives the opportunity to identify new repairing actions which introduce new knowledge to the ontology. An example of this is found in the BioTop experiment where is-a relations from the initial set of missing is-a relations were repaired by more informative solutions in the third iteration.

Currently, the system removes redundant is-a relations from a solution after every iteration. This step is crucial for producing skyline optimal solutions. However, in situations where an is-a relation is repaired by a relation acquired from the axioms containing $\exists$ expressions it might be advantageous to keep also the missing is-a relation in subsequent iterations even though it is redundant. The reason for this is that the Source set and the Target set for the missing is-a relation might get updated in later iterations and therefore new repairing actions might be identified. One way to solve this is to make it possible in the system to show these missing is-a relations with their Source and Target sets but not to include them in the solution unless they are repaired using new knowledge. For example, let us assume that the missing is-a relation Human $\sqsubseteq$ Primate was repaired in one iteration by a repairing action $\{$ Human $\sqsubseteq$ Primate, SpeciesHomoSapiensQuality $\sqsubseteq$ 
OrderPrimatesQuality in which case the second relation was found using $\exists$. In the next iteration the relation GreatApe $\sqsubseteq$ Primate was added to the ontology. If the system removed redundant relation Human $\sqsubseteq$ Primate then relation Human $\sqsubseteq$ GreatApe would not be detected as a possible repairing action for Human $\sqsubseteq$ Primate.

In cases where missing is-a relations are repaired using new knowledge, new is-a relations are added to the ontology which were not derivable before. These new is-a relations can be considered as missing is-a relations as they were not detected by the detection algorithm. Given this, the system can also be used for completing the is-a structure of ontologies, even when no missing is-a relations are available. This can be achieved by using a set of isa relations which are already derivable from the ontology as input. As in the BioTop experiment, by doing this, the system may identify additional is-a relations which represent new knowledge which can be added to the ontology. This methodology also allows a domain expert to deal with existing is-a relations which the domain expert has identified as relations which need to be revised or investigated further. 


\section{Chapter 5}

\section{Repairing missing is-a structure in $\mathcal{A L C}$ ontologies}

So far we have discussed repairing missing is-a structure (i.e. solving the GTAP) in the context of ontologies represented in $\mathcal{E} \mathcal{L}$ family of description logics. Our approach worked with normalized ontologies and used different logical patterns in order to identify solutions to the GTAP e.g. solutions acquired using $\exists$ statements.

In this chapter ${ }^{1}$, we focus on more expressive ontologies, more specifically ontologies represented in description logics $\mathcal{A L C}$. Description logic $\mathcal{A L C}$ is often considered a basic expressive description logic as it allows disjunction, concept negation and universal and existential quantifiers thus setting the foundation on which more expressive description logics are built [14].

Given the new logical constructors, computing Source and Target sets containing complex subconcepts and superconcepts of some concept as done in the $\mathcal{E} \mathcal{L}$ algorithm would require identification of a number of new logical patterns for dealing with the new logical constructors and their combinations. This would have be done for every more expressive language. Therefore, in this chapter we present a more general approach for solving GTAP which is based on satisfiability checking using tableaux reasoning. Our approach works directly on the completion graph, where it tries to identify is-a relations which would close the completion graph and therefore make a missing is-a relation satisfiable (derivable) in the ontology.

The chapter is organized in the following way. First, we propose an algorithm for solving GTAP in $\mathcal{A L C}$ ontologies that can be represented using acyclic terminologies (Section 5.1). Further, we discuss a system that allows a domain expert to repair missing is-a structure in Section 5.2. We discuss

\footnotetext{
${ }^{1}$ The chapter is a refined version of $[67]$
} 
the functionality and user interface of the repairing system and show an example run (Section 5.3). Finally, in Section 5.4 we discuss the evaluation of the system on two experiments from Chapter 4 as well as lessons learned.

\subsection{Algorithm}

The basic algorithm for repairing missing is-a structure (i.e. solving GTAP $(T, C, O r, M))$ in ontologies represented as acyclic $\mathcal{A L C}$ terminologies is shown in Algorithm 3. We require that the missing is-a relations are validated to be correct according to the domain i.e. $\forall m \in M: O r(m)=$ true as well as that $\mathrm{T} \cup \mathrm{M}$ is consistent.

Same as in the case of the $\mathcal{E} \mathcal{L}$ and the $\mathcal{E} \mathcal{L}^{++}$algorithms in Chapter 4 , the algorithm in Algorithm 3 computes a skyline optimal solution for a GTAP $(T, C, O r, M)$. Given that $M$ is a solution, the algorithm always returns a solution. This result can be a subset minimal solution which is a subset of $M$ or a solution which is more informative than $M$.

In the basic step of the algorithm (RepairSingleIsa) solutions for a GTAP with one missing is-a relation (i.e. $\operatorname{GTAP}(T, C, O r,\{E \sqsubseteq F\})$ are generated. This is done by first running the satisfiability checking algorithm with unfolding on demand as described in Section 2.3, on the knowledge base with input $E \sqcap \neg F$, and we collect the ABoxes of the leaves. As $E \sqsubseteq F$ is a missing is-a relation, it cannot be derived from $\mathrm{T}$ and thus the completion graph will have open leaves. We then generate different ways to close these ABoxes. For each individual $x$ in an open leaf ABox we collect the concepts in the statements of the form $x: P$ in $\operatorname{Pos}_{x}$ and the concepts in the statements of the form $x: \neg N$ in $\mathrm{Neg}_{x}$ where $\mathrm{P}$ and $\mathrm{N}$ are named concepts. The ABox can be closed if $\mathrm{P} \sqsubseteq \mathrm{N}$ is added to the ontology for any $\mathrm{P} \in \operatorname{Pos}_{x}$ and $\mathrm{N} \in \mathrm{Neg}_{x}$. Indeed, with this extra information $x: P$ could be unfolded and $x: N$ would be added to the ABox, and this gives a contradiction with $x: \neg N$ which was already in the ABox. Every relation $\mathrm{P} \sqsubseteq \mathrm{N}$ is checked if it is correct according to the domain and if the knowledge base extended with current solution and the relation is consistent (line 12). Next, the algorithm generates additional ways of closing open ABoxes. That is if $P \sqsubseteq N$ is used as one of the is-a relations in a solution then also $G \sqsubseteq H$ where $G$ is a superconcept of $A$ and $H$ is a subconcept of $B$ could be used. Therefore, the algorithm generates two sets of concepts for every is-a relation $P \sqsubseteq N$, Source set containing named superconcepts of $P$ and Target set containing named subconcepts of $N$. Again, it is necessary to check that these relations are correct according to the domain and that the extended knowledge base is consistent (line 18).

A solution to $\operatorname{GTAP}(T, C, O r,\{E \sqsubseteq F\})$ is a set of $\mathrm{P} \sqsubseteq \mathrm{N}$ that closes each open leaf ABox. If any of the ABoxes cannot be closed the algorithm would simply return the missing is-a relation (lines 20-23).

Finally, in line 24, redundancy is removed from the solution while keeping the same level of informativeness. Redundancy is removed in the same way 


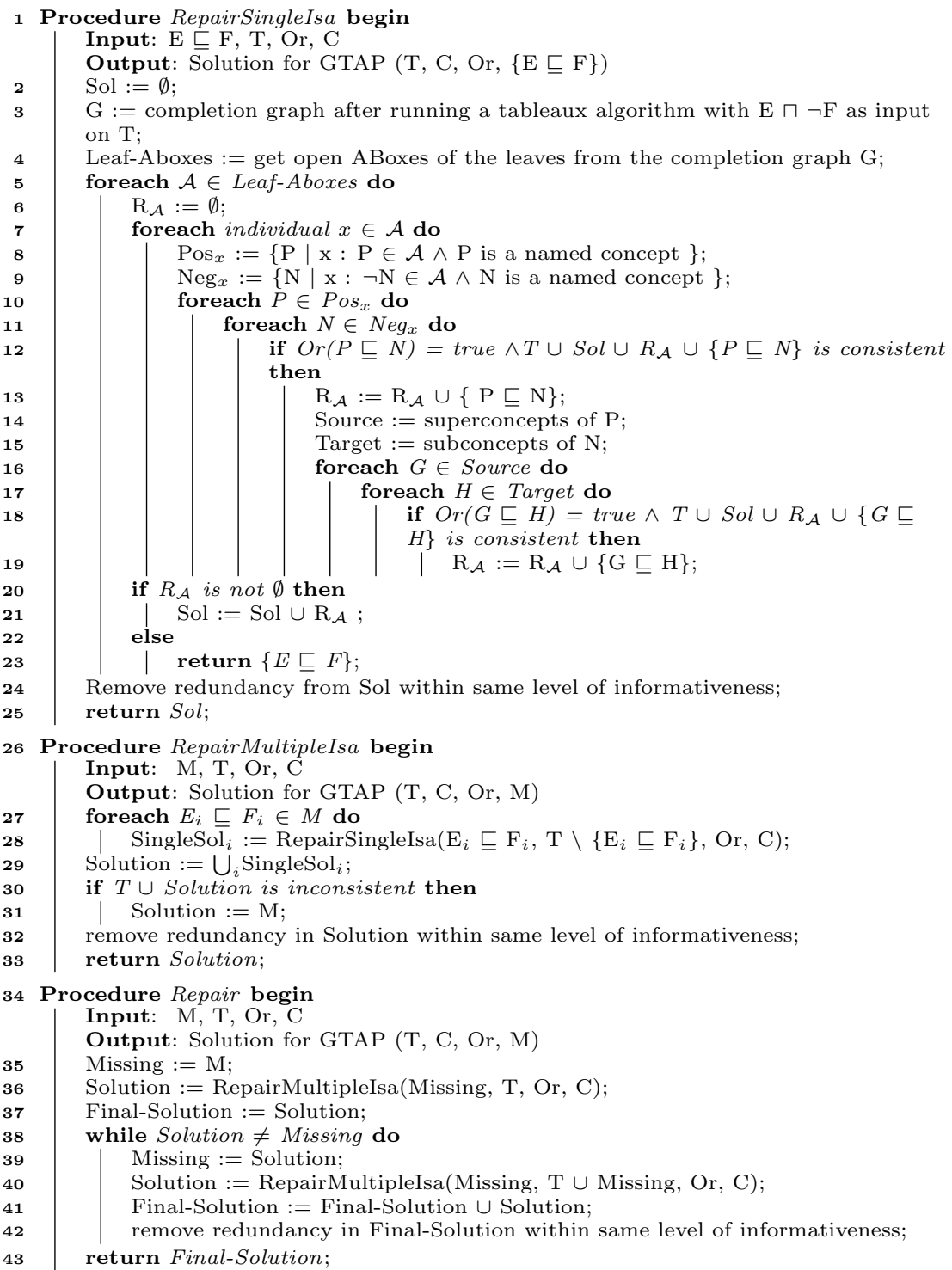

Algorithm 3: Algorithm for solving GTAP in $\mathcal{A L C}$.

as in the $\mathcal{E} \mathcal{L}$ and $\mathcal{E} \mathcal{L}^{++}$algorithms, i.e. in cases where there are multiple choices for removing redundancy, one of them is chosen.

The following holds for Sol returned by RepairSingleIsa: $T \cup S o l=$ $E \sqsubseteq F, \forall s \in$ Sol $: \operatorname{Or}(s)=$ true and $\mathrm{T} \cup \mathrm{Sol}$ is consistent.

In RepairMultipleIsa the solutions for each missing is-a relation acquired from RepairSingleIsa are combined. In order to find a solution for a missing is-a relation $\mathrm{E} \sqsubseteq \mathrm{F}$, the procedure RepairSingleIsa is run on a TBox from 
which $\mathrm{E} \sqsubseteq \mathrm{F}$ is removed. This removal is needed for the iterations as otherwise the relation would be derivable from the ontology and a completion graph without open ABoxes would be returned. This does not influence the soundness of the algorithm as the solution from RepairSingleIsa for $\mathrm{E} \sqsubseteq \mathrm{F}$ would at least include the missing is-a relation itself.

The union of solutions for each missing is-a relation represents a solution for $\operatorname{GTAP}(T, C, O r, M)$. The following holds for Solution in line 29: $T \cup$ Solution $=M$ and $\forall s \in$ Solution : $\operatorname{Or}(s)=$ true. Given the fact that $\mathcal{A L C}$ knowledge bases can be inconsistent, the algorithm contains a check for consistency (lines 30-31). If it happens that the solution is inconsistent, then the algorithm returns the set of missing is-a relations thus effectively terminating the algorithm. With this check the algorithm is sound and guarantees a solution (at least $\mathrm{M}$ is returned). In line 32, redundancy is removed from the solution to acquire a skyline optimal solution.

The idea of the Repair procedure is to try to improve the solutions returned by RepairMultipleIsa by finding a skyline optimal solutions on a higher level of informativeness. The solution returned by RepairMultipleIsa can be considered as a new set of missing is-a relations which then form a new GTAP problem. The (skyline optimal) solution to this new GTAP is also a (skyline optimal) solution to the original problem.

As an example run let us consider the GTAP in Figure 5.1. ${ }^{2}$ The first step of the algorithm is to identify solutions for individual missing is-a relations using RepairMultipleIsa.

For the missing is-a relation MyPizza $\sqsubseteq$ FishyMeatyPizza the algorithm starts by generating the completion graph by running the satisfiability checking algorithm on input MyPizza $\sqcap \neg$ FishyMeatyPizza (Figure 5.5 at the end of the chapter). Next, open leaf ABoxes are identified, and the possible ways of closing these ABoxes are generated. The sets $\mathrm{R}_{\mathcal{A}}$ for the open ABoxes in the completion graph are given in Figure 5.6 at the end of the chapter. Out of the is-a relations in $\mathrm{R}_{\mathcal{A}}$ sets, MyPizza $\sqsubseteq$ FishyMeatyPizza, AnchoviesTopping $\sqsubseteq$ FishTopping and ParmaHamTopping $\sqsubseteq$ MeatTopping are validated to be correct. These relations also appear in all $\mathrm{R}_{\mathcal{A}}$ sets and do not cause inconsistency when added to the knowledge base. In the next step, the algorithm tries to identify additional ways of closing open ABoxes. In our example, relation ParmaHamTopping $\sqsubseteq$ HamTopping is identified as an alternative for relation ParmaHamTopping $\sqsubseteq$ MeatTopping. The solution which closes all open ABoxes is MyPizza $\sqsubseteq$ FishyMeatyPizza, AnchoviesTopping $\sqsubseteq$ FishTopping, ParmaHamTopping $\sqsubseteq$ MeatTopping, ParmaHamTopping $\sqsubseteq$ HamTopping $\}$. From this solution relations MyPizza $\sqsubseteq$ FishyMeatyPizza and ParmaHamTopping $\sqsubseteq$ MeatTopping are removed in line 24 as they are redundant. Therefore, the procedure returns $\{$ AnchoviesTopping $\sqsubseteq$ Fish-

\footnotetext{
${ }^{2}$ We observe that the TBox in Figure 5.1 is not an acyclic terminology as there are statements of the form $A \sqsubseteq C$. However, it is possible to create an equivalent TBox that is an acyclic terminology by replacing the statements of the form $A \sqsubseteq C$ with $A \equiv C \sqcap$ $\bar{A}$ where $\bar{A}$ is new atomic concept. See Figure 5.2.
} 
$C=\{$ Pizza, PizzaTopping, MeatTopping, HamTopping, ProsciuttoTopping, ParmaHamTopping, FishTopping, TomatoTopping, GarlicTopping, AnchoviesTopping, MyPizza, FishyMeatyPizza, FruttiDiMare, VegetarianPizza, NonVegetarianPizza, ProsciuttoPizza\}

$T=\{$ AnchoviesTopping $\sqsubseteq$ PizzaTopping, MeatTopping $\sqsubseteq$ PizzaTopping, HamTopping $\sqsubseteq$ MeatTopping, FishTopping $\sqsubseteq$ PizzaTopping $\sqcap \neg$ MeatTopping, TomatoTopping $\sqsubseteq$ PizzaTopping $\sqcap \neg$ MeatTopping $\sqcap \neg$ FishTopping, GarlicTopping $\sqsubseteq$ PizzaTopping $\sqcap$ $\neg$ MeatTopping $\sqcap \neg$ FishTopping, ParmaHamTopping $\sqsubseteq$ PizzaTopping, ProsciuttoTopping $\sqsubseteq$ PizzaTopping,

$\bar{M} y$ Pizza $\equiv$ Pizza $\sqcap$ ヨhasTopping.AnchoviesTopping $\sqcap$ $\exists$ hasTopping.ParmaHamTopping,

FishyMeatyPizza $\equiv$ Pizza $\sqcap \exists$ hasTopping.FishTopping $\sqcap$ $\exists$ hasTopping.MeatTopping,

FruttiDiMare $\equiv$ Pizza $\sqcap \exists$ hasTopping.AnchoviesTopping

$\sqcap \exists$ hasTopping. GarlicTopping $\sqcap \exists$ hasTopping.TomatoTopping

$\sqcap \forall$ hasTopping.(AnchoviesTopping $\sqcup$ GarlicTopping $\sqcup$ TomatoTopping)

VegetarianPizza $\equiv$ Pizza $\sqcap \neg \exists$ hasTopping.FishTopping $\sqcap \neg \exists$ hasTopping. MeatTopping,

NonVegetarianPizza $\equiv$ Pizza $\sqcap \neg$ VegetarianPizza $\}$

$M=\{$ MyPizza $\sqsubseteq$ FishyMeatyPizza, FruttiDiMare $\sqsubseteq$ NonVegetarianPizza, ProsciuttoPizza $\sqsubseteq$ NonVegetarianPizza $\}$

The following is-a relations are correct according to the domain, i.e Or returns true for: MeatTopping $\sqsubseteq$ PizzaTopping, HamTopping $\sqsubseteq$ MeatTopping, HamTopping $\sqsubseteq$ PizzaTopping, ProsciuttoTopping $\sqsubseteq$ MeatTopping, ProsciuttoTopping $\sqsubseteq$ HamTopping, ProsciuttoTopping $\sqsubset$ PizzaTopping, ParmaHamTopping $\sqsubset$ MeatTopping, ParmaHamTopping $\sqsubset$ HamTopping, ParmaHamTopping $\sqsubseteq$ ProsciuttoTopping, ParmaHamTopping $\sqsubseteq$ PizzaTopping, FishTopping $\sqsubseteq$ PizzaTopping, AnchoviesTopping $\sqsubseteq$ FishTopping, AnchoviesTopping $\sqsubseteq$ PizzaTopping, TomatoTopping $\sqsubseteq$ PizzaTopping, GarlicTopping $\sqsubseteq$ PizzaTopping, VegetarianPizza $\sqsubset$ Pizza, NonVegetarianPizza $\sqsubset$ Pizza, ProsciuttoPizza $\sqsubset$ Pizza, ProsciuttoPizza $\sqsubset$ NonVegetarianPizza, FishyMeatyPizza $\sqsubseteq$ Pizza, FishyMeatyPizza $\sqsubseteq$ NonVegetarianPizza, MyPizza $\sqsubseteq$ Pizza, MyPizza $\sqsubseteq$ NonVegetarianPizza, FruttiDiMare $\sqsubseteq$ Pizza, FruttiDiMare $\sqsubseteq$ NonVegetarianPizza.

Let $\mathcal{P}=\operatorname{GTAP}(T, C, O r, M)$.

Figure 5.1: Small $\mathcal{A L C}$ example.

Topping, ParmaHamTopping $\sqsubseteq$ HamTopping\}.

The same process is repeated for the missing is-a relation FruttiDiMare $\sqsubseteq$ NonVegetarianPizza. For this relation, there were no new relations which needed to be validated, and the procedure returns \{AnchoviesTopping $\sqsubseteq$ FishTopping $\}$ while the relation FruttiDiMare $\sqsubseteq$ NonVegetarianPizza is removed as it is redundant.

Finally, for the third missing is-a relation ProsciuttoPizza $\sqsubseteq$ NonVegetarianPizza it was necessary to validate relations ProsciuttoTopping $\sqsubseteq$ FishTopping and ProsciuttoTopping $\sqsubseteq$ MeatTopping out of which only the latter is identified to be correct according to the domain. In addition relation ProsciuttoTopping $\sqsubseteq$ Ham Topping is identified as an additional way of closing the open ABoxes. The procedure returns \{ProsciuttoTopping $\sqsubseteq$ HamTopping\} as relation ProsciuttoTopping $\sqsubseteq$ MeatTopping is redundant.

In RepairMultipleIsa solutions for individual missing is-a relations are combined to form a solution for the set of missing is-a relations. Given that there are no redundant relations, RepairMultipleIsa returns \{ParmaHamTopping $\sqsubseteq$ MeatTopping, AnchoviesTopping $\sqsubseteq$ FishTopping, ProsciuttoTopping $\sqsubseteq$ HamTopping\}. We note that this is a skyline optimal solution.

In procedure Repair we try to improve the solution and find a new skyline 


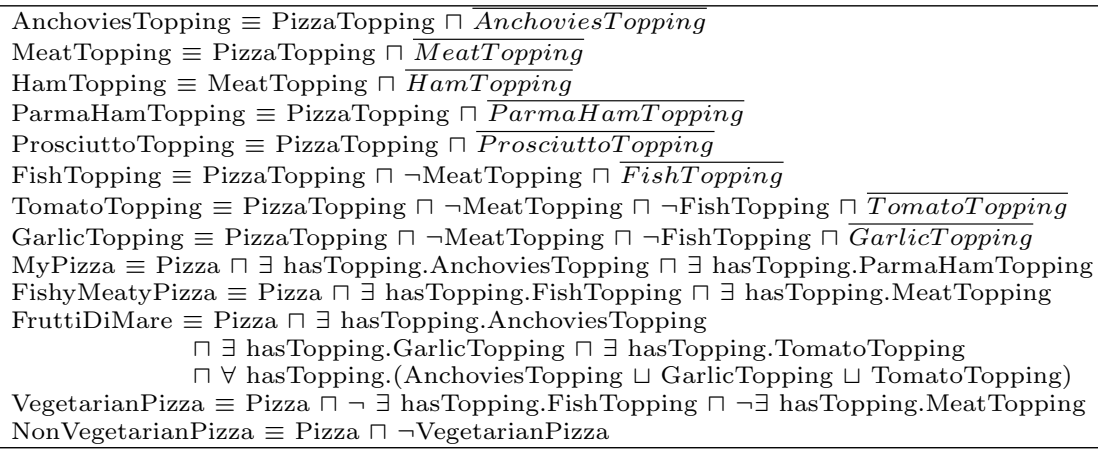

Figure 5.2: Tbox $\mathrm{T}$ from Figure 5.1 represented as an acyclic $\mathcal{A L C}$ terminology.

optimal solution on higher level of informativeness. However, this time the set of missing is-a relations is a solution from the previous iteration. Again, we run RepairSingleIsa on each individual missing is-a relation. However, given that the TBox was extended with the solution in Repair it is necessary to remove the missing is-a relation under repair from TBox when running RepairSingleIsa as we would get a closed completion graph. In our case the solutions for AnchoviesTopping $\sqsubseteq$ FishTopping and ProsciuttoTopping $\sqsubseteq$ HamTopping did not improve and RepairSingleIsa returns \{AnchoviesTopping $\sqsubseteq$ FishTopping\} and \{ProsciuttoTopping $\sqsubseteq$ HamTopping $\}$, respectively. In the case of ParmaHamTopping $\sqsubseteq$ MeatTopping, \{ParmaHamTopping $\sqsubseteq$ ProsciuttoTopping $\}$ is identified as a solution. This solution is possible given that in the previous iteration ProsciuttoTopping $\sqsubseteq$ HamTopping was added to the knowledge base so adding ParmaHamTopping $\sqsubseteq$ ProsciuttoTopping to the knowledge base would make ParmaHamTopping $\sqsubseteq$ HamTopping derivable.

In RepairMultipleIsa the solutions are combined to form the solution \{ParmaHamTopping $\sqsubseteq$ ProsciuttoTopping, ProsciuttoTopping $\sqsubseteq$ HamTopping, AnchoviesTopping $\sqsubseteq$ FishTopping $\}$ which is also a skyline optimal solution and on a higher level of informativeness compared to the solution from the first iteration.

Given that new is-a relations have been identified, the repair is run again, however in this iteration no new is-a relations were identified and the algorithm returns $\{$ ParmaHamTopping $\sqsubseteq$ ProsciuttoTopping, ProsciuttoTopping $\sqsubseteq$ HamTopping, AnchoviesTopping $\sqsubseteq$ FishTopping $\}$ and terminates. 


\subsection{System}

We have implemented a system that supports the user to repair missing is-a relations. The system was implemented in Java and uses Pellet (version 2.3.0) [89]. In our system the user first loads the ontology and the missing is-a relations from the File menu. The missing is-a relations are then shown in a drop-down list (e.g. MyPizza $\sqsubseteq$ FishyMeatyPizza ${ }^{3}$ in the second drop-down list in Figure 5.3). The repairing process is started by pressing the Generate Repairing Actions button. The system then generates solutions to GTAP for every individual missing is-a relation following the ideas in the RepairSingleIsA procedure in Algorithm 3. However, instead of producing a single solution as in the RepairSingleIsA procedure the system produces multiple subset minimal solutions. The user then needs to select at least one solution to repair the missing is-a relation and the system then combines selected solutions to produce a solution such as the one returned by the RepairSingleIsA procedure. Given this the system is easier to use as the user does not need knowledge of tableaux reasoning or of our approach for closing open ABoxes.

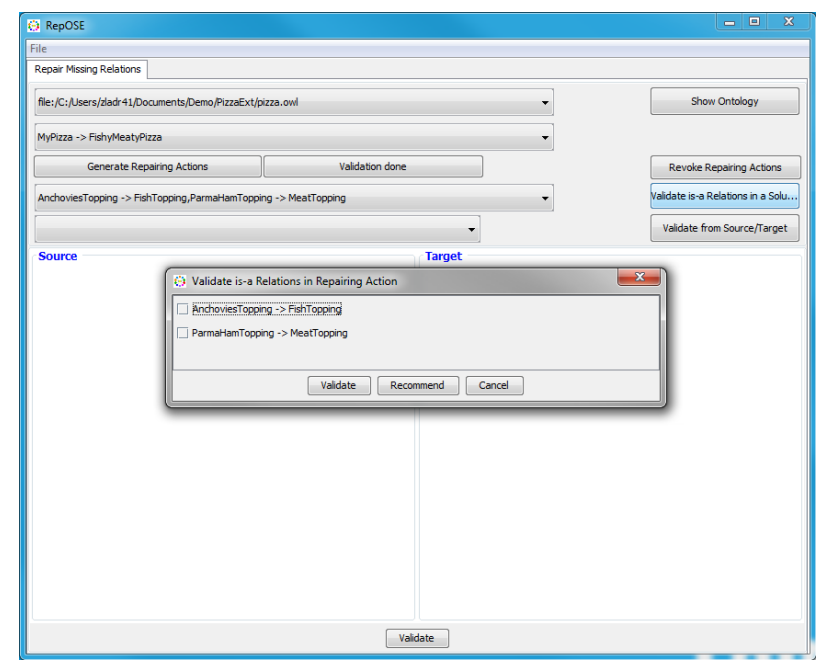

Figure 5.3: Screenshot - Validating is-a relations in a repairing action.

In order to acquire solutions, the satisfiability checker in Pellet was modified in order to extract full completion graphs. Furthermore, to increase performance and account for higher level of non-determinism, ontologies are first passed through Pellint [75] before running the algorithm. The computed GTAP solutions for a selected is-a relation are shown in the drop-down list

\footnotetext{
${ }^{3}$ In the system $\mathrm{C} \sqsubseteq \mathrm{D}$ is shown as $\mathrm{C} \rightarrow \mathrm{D}$.
} 
under buttons. Each solution consists of one or more is-a relations. Solutions marked in red represent solutions which would make the ontology incoherent.

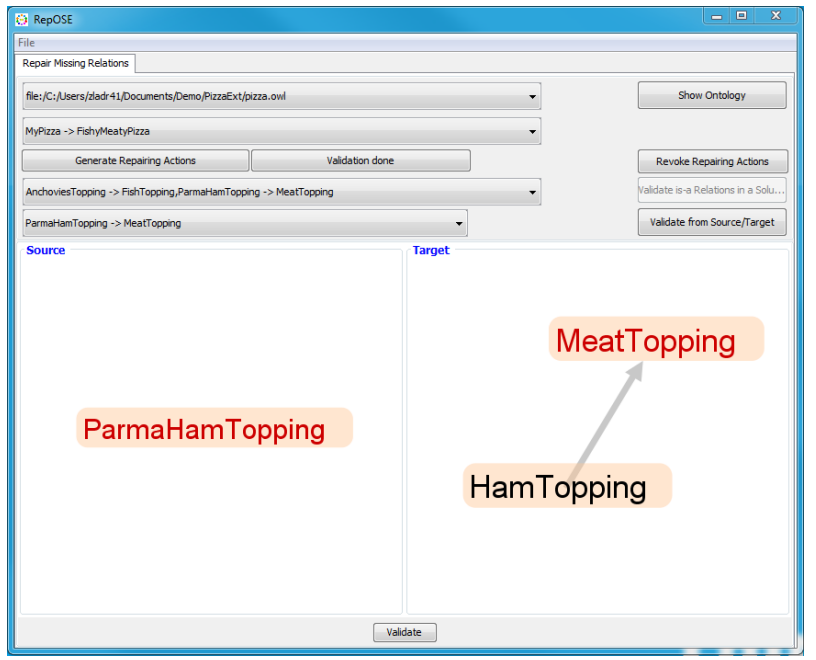

Figure 5.4: Screenshot - Repairing using Source and Target sets.

In Figure 5.3 the user has chosen to repair MyPizza $\sqsubseteq$ FishyMeatyPizza and the system has generated eleven possible solutions out of which $\{$ MyPizza $\sqsubseteq$ FishyMeatyPizza $\},\{$ AnchoviesTopping $\sqsubseteq$ FishTopping, ParmaHamTopping $\sqsubseteq$ MeatTopping\}, and AnchoviesTopping $\sqsubseteq$ MeatTopping, ParmaHamTopping $\sqsubseteq$ FishTopping $\}$ do not introduce incoherence.

As mentioned earlier, to repair a missing is-a relation the user has to successfully deal with at least one of the solutions, i.e. validate all is-a relations (or more informative is-a relations from Source and Target) as correct according to the domain in at least one of the generated solutions. The missing is-a relations are automatically validated to be correct according to the domain.

In Figure 5.3 the user has chosen $\{$ AnchoviesTopping $\sqsubseteq$ FishTopping, ParmaHamTopping $\sqsubseteq$ MeatTopping $\}$. When a solution is added to the ontology, it will make the missing is-a relation derivable. However, it is not guaranteed that all the is-a relations in the solution are also valid with respect to the domain. Therefore, a domain expert needs to validate the is-a relations in the repairing actions. When the user clicks the Validate is-a Relations in a Solution button, a pop-up window (Figure 5.3) appears where the user can mark all the is-a relations that are correct with respect to the domain model. Solutions for all missing is-a relations are updated according to the results of the validation. If an is-a relation is validated as incorrect according to the domain, all solutions that contain this incorrect 
is-a relation, for this and for all other missing is-a relations, are removed from the lists of the solutions. When an is-a relation is validated as correct it is marked as correct in all repairing actions for all missing is-a relations.

When all is-a relations in the current solution are validated as correct, they are shown in the last drop-down list (Figure 5.4). ${ }^{4}$ Now the user can repair them one by one.

For each is-a relation within the solution which is validated to be correct according to the domain the system generates the Source and Target sets displayed on the left and the right hand sides, respectively, within the panel under the drop-down lists (Figure 5.4). Using Source and Target set the user can identify additional is-a relations which would make the selected is-a relation derivable as well as introduce new knowledge to the ontology. Both panels have zoom control and can be opened in a separate window. The concepts in the is-a relation under consideration are highlighted in red, existing asserted and inferred is-a relations are shown in grey. The system implements the same optimization as the $\mathcal{E} \mathcal{L}$ algorithms which disallows introduction of non-validated equivalence relations where in the original ontology there are only is-a relations.

In order to validate an additional is-a relation the user has to choose one concept from each of the sets and click the Validate button. The user can also click the Validate from Source/Target and validate is-a relation on the pop-up window.

In Figure 5.4 the user has chosen to validate ParmaHamTopping $\sqsubseteq$ HamTopping for the is-a relation ParmaHamTopping $\sqsubseteq$ MeatTopping from the solution \{AnchoviesTopping $\sqsubseteq$ FishTopping, ParmaHamTopping $\sqsubseteq$ HamTopping\}.

We end the validation phase by clicking the Validation Done button. The system then proceeds by calculating consequences of the selected repairing actions and computes a new set of missing is-a relations which is then presented to the user. The validation phase and consequent computations represent one iteration of the Repair procedure in Algorithm 3. If there are no changes in the repairing between two iterations, the completing process is done and the system outputs the repairing.

Same as in the $\mathcal{E} \mathcal{L}$ system, the user can at any time save or load validated is-a relations from the File menu and therefore execute the debugging process across multiple sessions.

\subsection{Example run}

As an example run, consider the ontology in Figure 5.1 and missing is-a relations MyPizza $\sqsubseteq$ FishyMeatyPizza, FruttiDiMare $\sqsubseteq$ NonVegetarianPizza and ProsciuttoPizza $\sqsubseteq$ NonVegetarianPizza. After loading the ontology and

\footnotetext{
${ }^{4}$ As there always is one solution that contains only the missing is-a relation, there will always be at least one solution for which all is-a relations are validated to be correct.
} 
the missing is-a relations, we can choose a missing is-a relation to repair. Assume we choose FruttiDiMare $\sqsubseteq$ NonVegetarianPizza and click the Generate Repairing Actions button. The system will show eleven solutions in the drop-down list: $\{$ FruttiDiMare $\sqsubseteq$ NonVegetarianPizza $\},\{$ AnchoviesTopping $\sqsubseteq$ FishTopping $\},$ GarlicTopping $\sqsubseteq$ MeatTopping\}, \{AnchoviesTopping $\sqsubseteq$ MeatTopping $\}$, \{GarlicTopping $\sqsubseteq$ FishTopping $\}$, \{TomatoTopping $\sqsubseteq$ FishTopping\}, \{TomatoTopping $\sqsubseteq$ MeatTopping\}, \{PizzaTopping $\sqsubseteq$ FishTopping $\},\{$ Pizza $\sqsubseteq$ NonVegetarianPizza $\},\{$ PizzaTopping $\sqsubseteq$ MeatTopping $\}$ and $\{$ VegetarianPizza $\sqsubseteq$ NonVegetarianPizza\}. Out of these FruttiDiMare $\sqsubseteq$ NonVegetarianPizza $\},\{$ AnchoviesTopping $\sqsubseteq$ FishTopping $\}$, and $\{$ AnchoviesTopping $\sqsubseteq$ MeatTopping $\}$ do not introduce incoherence. We can then choose to deal with $\{$ AnchoviesTopping $\sqsubseteq$ MeatTopping $\}$ and validate the relation to be incorrect with respect to the domain. In this case all solutions containing this is-a relation will be removed. We could then choose \{AnchoviesTopping $\sqsubseteq$ FishTopping $\}$ and validate it to be correct. All is-a relations in this solution (i.e. AnchoviesTopping $\sqsubseteq$ FishTopping) are validated to be correct and thus the system generates the Source and Target sets for AnchoviesTopping $\sqsubseteq$ FishTopping. In this small example the Source set only contains AnchoviesTopping and the Target set only contains FishTopping. Given that this relation has already been validated it is marked in the pop-up window.

We then proceed by repairing MyPizza $\sqsubseteq$ FishyMeatyPizza. The system would have generated eight possible solutions: \{MyPizza $\sqsubseteq$ FishyMeatyPizza\}, \{AnchoviesTopping $\sqsubseteq$ FishTopping, ParmaHamTopping $\sqsubseteq$ MeatTopping $\}$, \{Pizza $\sqsubseteq$ FishyMeatyPizza $\},$ P PizzaTopping $\sqsubseteq$ FishTopping, PizzaTopping $\sqsubseteq$ MeatTopping $\}$, \{ParmaHamTopping $\sqsubseteq$ MeatTopping, ParmaHamTopping $\sqsubseteq$ FishTopping $\}$, \{ParmaHamTopping $\sqsubseteq$ MeatTopping, PizzaTopping $\sqsubseteq$ FishTopping $\},$ ParmaHamTopping $\sqsubseteq$ FishTopping, PizzaTopping $\sqsubseteq$ MeatTopping $\}$ and AnchoviesTopping $\sqsubseteq$ FishTopping, PizzaTopping $\sqsubseteq$ MeatTopping $\}$. Out of these only $\{$ MyPizza $\sqsubseteq$ FishyMeatyPizza $\}$ and AnchoviesTopping $\sqsubseteq$ FishTopping, ParmaHamTopping $\sqsubseteq$ MeatTopping\} do not introduce incoherence. Given that we earlier validated AnchoviesTopping $\sqsubseteq$ MeatTopping to be incorrect with respect to the domain, three solutions containing this relation are not shown to the user: \{AnchoviesTopping $\sqsubseteq$ MeatTopping, ParmaHamTopping $\sqsubseteq$ FishTopping\}, \{AnchoviesTopping $\sqsubseteq$ MeatTopping, AnchoviesTopping $\sqsubseteq$ FishTopping $\}$ and \{AnchoviesTopping $\sqsubseteq$ MeatTopping, PizzaTopping $\sqsubseteq$ FishTopping\}.

When we choose the second solution, AnchoviesTopping $\sqsubseteq$ FishTopping is already marked as correct (because of earlier validation) and only ParmaHamTopping $\sqsubseteq$ MeatTopping needs to be validated. We validate this as correct and then the system generates Source and Target sets. The Source set in this case contains ParmaHamTopping and the Target set contains HamTopping and MeatTopping. In this case ParmaHamTopping $\sqsubseteq$ MeatTopping is already validated however it is more informative (and correct with respect to the domain) to add ParmaHamTopping $\sqsubseteq$ HamTopping. 
We therefore choose the latter. All is-a relations in this solutions are validated and thus MyPizza $\sqsubseteq$ FishyMeatyPizza is repaired.

Finally, for the missing is-a relation ProscuittoPizza $\sqsubseteq$ NonVegetarianPizza there are seven solutions: \{ProsciuttoTopping $\sqsubseteq$ FishTopping\}, \{ProsciuttoTopping $\sqsubseteq$ MeatTopping\}, \{ProscuittoPizza $\sqsubseteq$ NonVegetarianPizza\}, \{PizzaTopping $\sqsubseteq$ FishTopping $\},$ PPizzaTopping $\sqsubseteq$ MeatTopping\}, $\{$ Pizza $\sqsubseteq$ NonVegetarianPizza $\}$ and $\{$ VegetarianPizza $\sqsubseteq$ NonVegetarianPizza\}. From these solutions, only first three do not introduce incoherence. In this case we select $\{$ ProsciuttoTopping $\sqsubseteq$ MeatTopping $\}$ and validate the relation as correct. In the generated Source and Target set we can additionally validate ProsciuttoTopping $\sqsubseteq$ HamTopping.

At this point we can end the iteration. The system then calculates the solution for the set of missing is-a relations by combining solutions for individual missing is-a relations and removing redundancy while keeping the same level of informativeness. In our case the solution would be AnchoviesTopping $\sqsubseteq$ FishTopping, ParmaHamTopping $\sqsubseteq$ HamTopping, ProsciuttoTopping $\sqsubseteq$ HamTopping\} which is a skyline optimal solution.

The solution is then used as input to the next iteration. In the next iteration AnchoviesTopping $\sqsubseteq$ FishTopping has two possible solutions \{AnchoviesTopping $\sqsubseteq$ FishTopping $\}$ and $\{$ PizzaTopping $\sqsubseteq$ FishTopping $\}$. The is-a relation in the first solution is already validated to be correct according to the domain and we can validate the is-a relations in the second to be incorrect according to the domain thus removing the solution from the list. The Source and Target sets for AnchoviesTopping $\sqsubseteq$ FishTopping only contain AnchoviesTopping and FishTopping respectively so no additional is-a relations can be validated. We have the similar situation for the missing is-a relation ProsciuttoTopping $\sqsubseteq$ HamTopping in which case again we have solutions, \{ProsciuttoTopping $\sqsubseteq$ HamTopping $\}$ and $\{$ PizzaTopping $\sqsubseteq$ HamTopping $\}$. In this case, PizzaTopping $\sqsubseteq$ HamTopping is incorrect according to the domain so we validate it as such and use the solution \{ProsciuttoTopping $\sqsubseteq$ HamTopping $\}$. No additional is-a relations can be validated from Source and Target sets.

Finally, for the missing is-a relation ParmaHamTopping $\sqsubseteq$ HamTopping we have only one possible solution i.e. $\{$ ParmaHamTopping $\sqsubseteq$ HamTopping\}. In this case the Source set for the is-a relation ParmaHamTopping $\sqsubseteq$ HamTopping contains ParmaHamTopping while the Target set contains HamTopping and ProsciuttoTopping. Given that ParmaHamTopping $\sqsubseteq$ ProsciuttoTopping is correct according to the domain we validate this isa relation as correct.

After this, we can end the iteration and the solution for the whole set of missing is-a relations is $\{$ AnchoviesTopping $\sqsubseteq$ FishTopping, ParmaHamTopping $\sqsubseteq$ ProsciuttoTopping, ProsciuttoTopping $\sqsubseteq$ HamTopping $\}$ which is again a skyline optimal solution however on a higher level of informativeness compared to the solution from the first iteration.

The system runs again a new iteration using the solution from the pre- 
vious iteration. However, in this iteration no new is-a relations could be validated and the system outputs the solution from the previous iteration as the final solution.

\subsection{Experiments}

We have run the same experiments from Section 4.4 on the $\mathcal{A L C}$ system. In the case of the two anatomy ontologies the computation of solutions took more time than with the $\mathcal{E} \mathcal{L}$ system. Initial computation of solutions took about 1 hour for the AMA ontology and around 16 hours for the NCI-A anatomy ontology. Inspection of the system has pointed that the majority of time needed for the computation of the solutions is spent on running a reasoner as a part of checking if a solution would introduce an incoherence. The checks are done by adding each solution to an ontology, running a reasoner on the extended ontology and then removing the solution from the ontology. If a solution is introducing an incoherence, then it is marked red in the graphical user interface. After removing the coherence check, the computation time was reduced to 20 minutes for AMA and about 1 hour for NCI-A case. The computation time between iterations without coherence check was around 1 hour and around 2 hours for AMA and NCIA, respectively. The majority of time spent on the computation between iterations was in relation to redundancy checking where the idea is to acquire skyline optimal solutions. The redundancy check is done by adding all solutions for individual missing is-a relation and then removing one is-a relation at a time and checking if the extended ontology still derives the removed is-a relation. If this is the case, then the is-a relation is redundant.

In both experiments the same solutions were acquired as in the $\mathcal{E} \mathcal{L}$ experiments. The $\mathcal{A L C}$ system offered more solutions in the experiments, however a large portion of these solutions would introduce incoherence into the ontology. An interesting observation is that in cases where missing is-a relations had more informative solutions, these solutions were in most cases offered as possible solutions without the need for generating Source and Target set for the missing is-a relation as done in the $\mathcal{E} \mathcal{L}$ system. These solutions are a result of unfoldings in the completion graph. However, given that in our case unfolding is done on demand, not all possible sub- and superconcepts are unfolded. Therefore, by generating Source and Target set we can acquire solutions which are on a higher level of informativeness. However, the fact that these solutions are returned by the system, in practice implies that the user does not need to deal with the full Source and Target sets but can restrict them by selecting a solution which is correct according to the domain and is more informative than just adding a missing is-a relation itself. The system was responsive after computation of solutions and no major slow-downs were encountered.

In the case of the BioTop experiment, the ontology contained a number of cycles in the axioms related to the missing is-a relations. Given that 
cycles are not supported by our $\mathcal{A L C}$ system the full experiment could not be done. However, a number of observations about the system can be made from partial results acquired from the system. First, there were a number of missing is-a relations in which case the completion graph contained many disjunctions which accounts to deep and bushy completion graphs. This is in contrast to the two anatomy experiments which in most cases produced completion graphs with a single leaf node. In cases where graphs contained many disjunctions the computation of solutions took multiple hours given that our approach for finding solutions in completion graphs generates all possible combinations for closures of open ABoxes. In addition, a large number of possible solutions were generated in these cases. The large number of solutions (more than 1000) induced a slow-down on the graphical user interface. Moreover, in practice this number of solutions is too large for a domain expert to handle and some form of filtering is needed.

\subsubsection{Lessons learned}

We have presented our approach for repairing missing is-a structure in $\mathcal{A L C}$ ontologies. Compared to the approach for repairing missing is-a structure in $\mathcal{E} \mathcal{L}^{++}$ontologies, the presented approach is more general and is applicable to more expressive description logics. Analysis of our approach and system has pinpointed possible points for improvement.

Our approach is based on a tableaux reasoning algorithm and uses the completion graph to calculate solutions to GTAP. Existing reasoners based on tableaux reasoning are optimized and often do not produce the whole completion graph as identifying one open ABox is enough to prove unsatisfiability. In our approach we require the full completion graph which represents the worst case scenario in satisfiability checking as all branches of a completion graph need to be checked. Further, when extracting solutions to GTAP from a completion graph, our approach makes all possible combinations for closure of open ABoxes which is computationally demanding. Given this, our system is unable to deal with larger more expressive ontologies. Closer inspection of this has shown that, it is the generation of all possible combinations of completion graph closures which is computationally more expensive than generating the full completion graph. One way to solve this scaling issue is to consider only some combinations of closures for solutions. This can be done by limiting the depth to which we consider ABox closures.

In the current approach, a large number of computed solutions are those which introduce new equivalence relations into the ontology. The number of solutions can be restricted by employing the same strategy as done with Source and Target sets where solutions which would introduce equivalence relations are not considered.

In situations where an inconsistency is detected in the solution, the solution from the previous iteration is returned in the case of $\mathcal{E} \mathcal{L}^{++}$and $\mathcal{A L C}$ 
algorithms for solving GTAP. As a result, all correctly validated relations from the current iteration will be lost and will not be considered in the solution. There a number of different approaches for dealing with this issue. The simplest approach is to retain partial solutions which do not cause inconsistencies. These partial solutions might contain new relations which are correct according to the domain and represent new correct knowledge which should be added to an ontology. Another approach is to incorporate approaches for dealing with semantic defects into our approach. This would mean that when an inconsistency is detected that we first remove the cause of the inconsistency and then continue with our approach for solving GTAP.

As discussed, the coherence checks induce a slow-down in the computation of solutions. One way of dealing with this is to provide coherence checking on demand. In other words, the user can select if he desires to check which solutions introduce incoherence. 


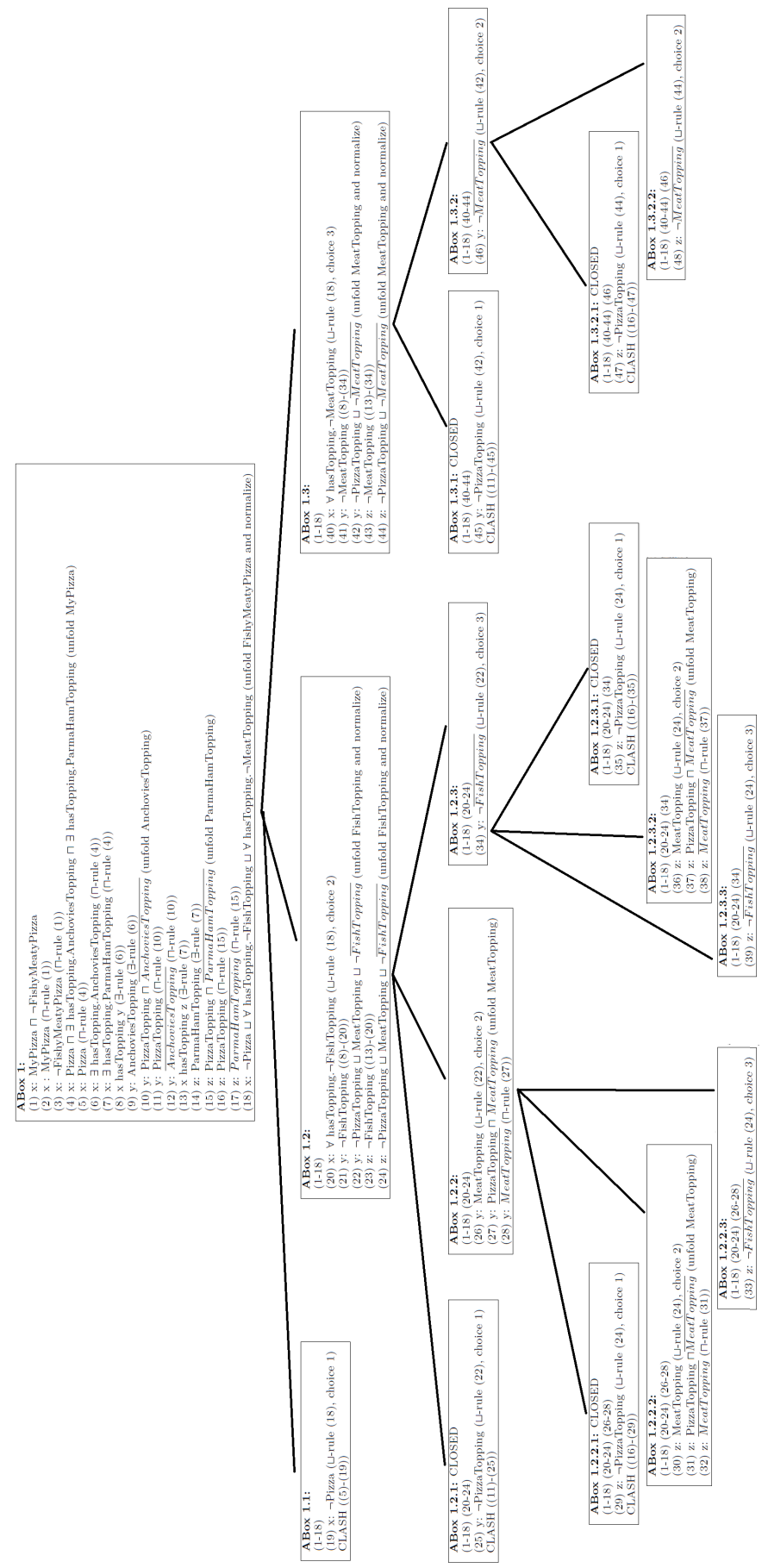

Figure 5.5: Completion graph for MyPizza $\neg$ FishyMeatyPizza. 


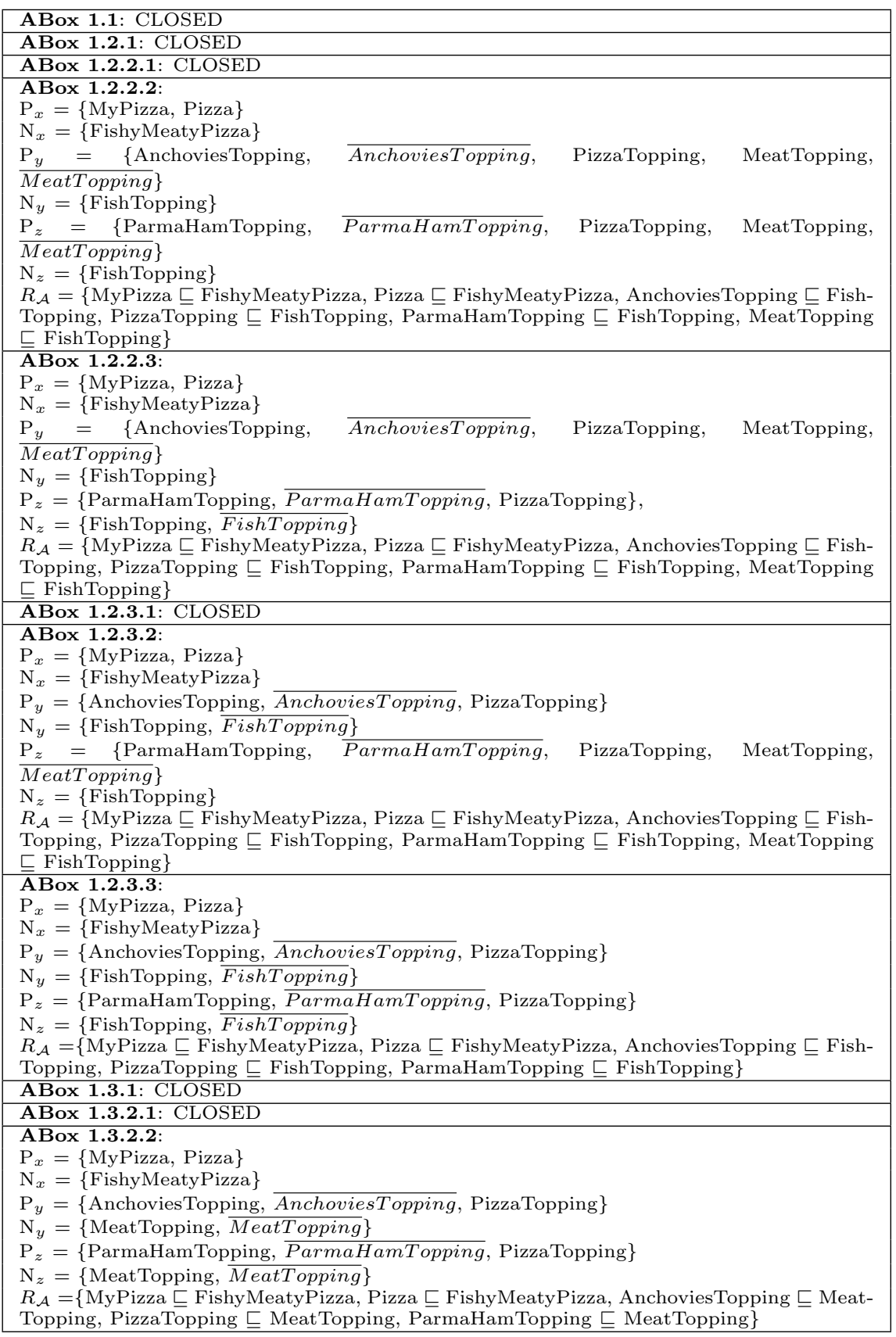

Figure 5.6: Creating $R_{\mathcal{A}}$ for the leaf ABoxes related to MyPizza $\sqsubseteq$ FishyMeatyPizza. 


\section{Chapter 6}

\section{Related work}

This chapter discusses related work in completing missing is-a structure in ontologies as well as state-of-the-art work in related topics such as detection of missing is-a relations, debugging semantic defects and abduction in description logics.

\subsection{Completing ontologies}

There is not much work dealing with completing of missing is-a structures. In [71] and [70] this problem was addressed in terms of taxonomies. In [71] the authors defined the problem of completing the is-a structure (called structural repairing in [71]). The defined problem can be seen as an abduction problem, however the authors' problem definition does not consider the role of a domain expert. The authors also introduced preference relations: axiom-preferred, information-preferred and strict-hierarchy-preferred. The axiom-preferred relation prefers repairs which do not contain redundant information, information-preferred prefers repairs which are more informative and strict-hierarchy-preferred prefers repairs which do not change is-a relations in a taxonomy into equivalence relations. The most axiom-preferred solutions would relate to a subset minimal solution in our setting. More informative solutions in our setting are a more strict version of informationpreferred solution as their work considers only is-a hierarchies. The authors introduced an algorithm for generating repairs based on Source and Target sets with respect to the introduced preference relations. An extension of the algorithm was proposed which considers influences of repairs for other missing is-a relations when repairing a missing is-a relation. In both cases, the user can only select a single relation per missing is-a relation. The approach is non-iterative and there is no guarantee that the acquired final solution is skyline optimal as it might not be subset minimal. The paper also discusses an approach for recommending repairing actions which aims at minimizing the interaction with background knowledge. The algorithms 
were implemented in a system which was evaluated on AMA ontology and NCI-A ontology.

The work in [71] focused on repairing a single ontology. The authors extended their work in [70] by considering repairing missing is-a structure in taxonomies which are a part of taxonomy networks, i.e. taxonomies networked by alignments. In addition to extending algorithms for generating, recommending and executing repairing actions to deal with taxonomies in this setting, the extension also considers a problem of detecting missing isa relations. In this case, candidate missing is-a relations are is-a relations which are derivable from a taxonomy network but are not derivable from a taxonomy itself. The candidate missing is-a relations then need to be validated by a domain expert to acquire a set of missing is-a relations.

We note that the approaches presented in this thesis for completing isa structure can be used for completing taxonomies and in that case our approach finds more informative solutions than [70, 71].

The work [68] extends further the previous work [70] and presents a unified approach for repairing missing and wrong is-a relations as well as missing and wrong mappings in networked taxonomies. The implemented system was evaluated on a number of taxonomies as well in a use case [55].

An unified approach for aligning and repairing taxonomies was presented in [54]. In this work, the authors have integrated their approach for repairing missing and wrong is-a relations and mappings with an alignment component. The alignment component is used for detecting additional candidate missing mappings. The candidate missing mappings are then validated and validated missing mappings are passed to the repairing component for repair.

\subsection{Detecting missing is-a relations}

There are different approaches for detecting missing is-a relations. A number of these approaches deal with detecting missing is-a relations in ontology networks. An approach in [17] discusses a way of enriching Open Biomedical Ontologies (OBO). This approach deals with subject-property-object terms in these ontologies and introduces the concept of nonalignments i.e. a subset of terms which have a subsumption relation between subjects but not between objects. This could be an implication that there are missing is-a relations between objects of terms in an nonalignment. Therefore, the next step is to validate using domain knowledge if this is the case. If so, missing is-a relations need to be repaired. The authors propose two ways for repairing a nonalignment by either adding is-a relations between objects or by removing is-a relations between subjects. As discussed in the previous section, a similar approach was used for detecting missing is-a relations [70] and for detecting both missing is-a relations and missing mappings in taxonomies $[55,54]$.

In [21] the authors discuss and compare two different methods for aligning NCI-A and AMA ontologies, a lexical approach and a manual alignment. 
In the lexical approach, the first step is to identify mappings by matching pairs of concepts which lexically match. After that, a structural validation is executed which compares hierarchical relations among concepts in detected mappings across ontologies. In other words, if two concepts that appear in two mappings are related in one ontology then the concepts to which they map should also be related in the other ontology. If this is not the case, the mapping needs to be removed. Structural validation can also be used as a way of identifying candidate missing is-a relations. The pairs of concepts from mappings which cannot be structurally validated are candidate missing is-a relation.

Some approaches for detecting modeling defects focus on properties of the is-a relation. The authors in [44] suggest that the main reasons why taxonomic information in ontologies is often complicated and difficult to understand is due to is-a overloading, i.e., linguistic definitions and properties of the is-a relation do not always reflect the ontological is-a relation. Therefore the author defines a number of ontology design principles which can be used to detect modeling defects in an ontology.

In [62] the author proposes methods for detecting and repairing flaws in object property expressions. Examples of flaws are domain and range flaws, property characteristics flaws and property chain issues. The author defines two services for debugging these flaws, one dealing with property hierarchies and the other one dealing with property chains. The detection in these services is based on a number of tests where property hierarchies and property chains are tested on. In addition, the author also specifies a number of guidelines on how to resolve situations where some test fails i.e. approaches for revising an ontology which include changes in property expressions or class hierarchy.

Some approaches from the ontology learning field for detecting relations between concepts in ontologies can be applied to detecting missing is-a relations. Ontology learning from text is discussed in [23]. The authors cover a number of methods and applications for extracting ontology relations from text corpora. The methods in this area typically build upon established methods from computational linguistics and natural language processing.

There are methods in computational linguistics which specifically deal with the detection of is-a relations. The work done in [50] specifies a number of lexico-syntactic patterns for detecting hyponym relations in a text corpus. The specified patterns describe relationships between noun phrases. An example of a simple pattern is " $N P_{0}$ such as $N P_{1}$ and $N P_{2}$ " in which case $N P_{1}$ and $N P_{2}$ could be identified as hyponyms of $N P_{0}$. The author also describes a procedure for detecting new patterns where the idea is to first identify related terms given some lexical relation and then try to generalize parts of text where the detected related terms appear close to each other. While these types of patterns have a high precision, their recall is usually low.

In [11] the authors propose three linguistic-based strategies for detecting 
relations between concepts in mappings. The first one, compound strategy, suggests that a concept A subsumes another concept B if the name of B is a compound word which ends with the name of A. The other strategy is based on background knowledge. Background knowledge sources such as thesauri and dictionaries are queried for linguistic relationships between concept names. The relationships are then used to decide relations between concepts in an ontology, e.g. synonym relation relates to equivalence or hyponym relation to subsumption. The third strategy is the itemization strategy which suggests that there exists an is-a relation between an itemization and items that it contains.

There are number of methods for detecting relations between concepts in ontologies based on machine learning techniques and statistical methods.

In [77] the authors discuss methods for extracting non-taxonomic relations from text based on association analysis. The input to the association analysis is a set of concept pairs which are acquired from a text processing component which associates concepts based on their linguistic dependency or heuristic correlations, e.g. concepts are coupled if they appear in the same sentence or prepositional phrases are coupled with adjacent noun phrases.

A supervised classification-based method for aligning ontologies is presented in [90]. The features used in the classification algorithm take into account both properties of textual descriptions of concepts (such as names, labels and comments) as well as properties of textual descriptions of related concepts, i.e., concepts related via equivalence, disjointness or subsumption axioms.

In [98] an approach for learning ontologies using probabilistic topic models is discussed. The approach uses Latent Dirichlet Allocation (LDA) [20] to identify topics from documents. The identified topics correspond to concepts in the new ontology. Conditional independence tests are then applied on identified topics to detect taxonomic relations.

In [24] the authors present a method for learning taxonomic information from text based on Formal Concept Analysis (FCA) [41]. The input to the FCA are verb-subject, verb-object and verb-prepositional phrase pairs which are acquired in a pre-processing phase. The output lattice from the FCA is transformed into a compacted partial order which corresponds to the resulting concept hierarchy.

Change management techniques from the ontology evolution area [49] can be used for detection of missing is-a relations. In [83] the authors discuss a process of adapting mappings in evolving ontologies. The approach for adapting mappings is based on a categorization of ontology changes. The authors specified three algorithms which deal with mapping adaptation after removal, addition or revision of knowledge in an ontology. 


\subsection{Debugging semantic defects}

Most of the work for debugging semantic defects aims at identifying and removing logical contradictions from an ontology. Standard reasoners are used to identify the existence of a contradiction, and provide support for resolving and eliminating it [40].

In [86] and [87] minimal sets of axioms are identified which need to be removed to render an ontology coherent. This is done using the pinpointing algorithm. The idea behind the algorithm is that those axioms which are responsible for the incoherences should be removed with minimal intrusion in the ontology. In order to find these axioms the algorithm first needs to find a minimal unsatisfiability-preserving sub-TBox (MUPS). A MUPS with respect to unsatisfiable concept A represents a minimal subsets of TBox which contains A and is unsatisfiable. Generated MUPSs for all unsatisfiable concepts are used in the next step for the calculation of minimal incoherence preserving sub-TBoxes (MIPSs). MIPSs correspond to the minimal sets of axioms which cause the incoherence in the TBox. After finding the set of MIPS in the ontology, the decision on which axioms to remove has to be made. Therefore, in [86] the author introduces the concept of cores which represent sets of axioms which appear in one or more MIPSs. The number of MIPSs where some core appears represents an arity of a given core. The cores are then used by the algorithm to identify a set of pinpoints, i.e., axioms which need to be removed from an ontology to make it coherent.

Kalyanpur et al. [60] proposed two approaches for debugging unsatisfiable concepts, glass-box techniques and black-box techniques. The glass-box techniques can provide information about clashes (causes of defects) as well as a set of axioms which caused the clash. The clash information provide the user with information needed for debugging defects. The information usually includes names of concepts participating in the clash. The information about axioms participating in the clash is provided in the sets of support. These sets are formed during the execution of a reasoner. The reasoner needs to be extended in such way that it keeps extra information which in the case of clash detection can be used to track the source axiom. The problems with the glass-box techniques are that they require reasoners which are capable of generating explanations and the memory overhead needed for dependency tracking.

The black-box techniques do not have these requirements. The main idea of black-box techniques is to divide unsatisfiable concepts into root concepts (those which are not dependent on any other unsatisfiable concept) and derived concepts (unsatisfiable concepts dependent on some other unsatisfiable concepts). The algorithm for searching for root/derived concepts is divided into two steps: detection of asserted dependencies and detection of inferred dependencies. The first step focuses on finding dependencies between unsatisfiable concepts among assertions within the ontology while the detection of inferred dependencies searches for dependencies among inferred structures 
of the ontology.

However, the presented techniques only provide the user with information which helps the user in understanding defects in an ontology. The actual repairing is done manually. The authors extended their work [59] proposing an approach for debugging unsatisfiable concepts similar to pinpointing [86, 87]. Their algorithm is based on precise MUPS, an extension of MUPS, which describes which specific parts of an axiom make the axiom unsatisfiable. To get the precise MUPS, the search for the MUPS is done on a new knowledge base which is made by splitting axioms across conjunctions into shorter and simpler axioms. The suitable axioms for removal are acquired using a modified version of the hitting set algorithm [84] which takes into account a ranking of an axiom. The authors present a number of different ranking approaches, such as impact of removal, annotation information, number of appearances of an axiom in an ontology, etc. In order to optimize the repairing process the authors reused the idea of root and derived unsatisfiable concepts [60]. The idea is that by repairing the root concepts a large portion of unsatisfiable concepts which were dependent on these concepts would also be repaired.

In [45] the focus is on maintaining the consistency as the ontology evolves. This is based on a formalization of the semantics of change for ontologies. Three types of consistency are formally defined: structural consistency, logical consistency and user-defined consistency. The authors also discuss resolution strategies for each type of corresponding inconsistency. In the case of logical consistency, two alternatives for resolution are discussed. The first alternative is to identify a consistent ontology for a given change by removing one axiom at a time from an inconsistent ontology. The second alternative suggests that the resolution is done by localizing the inconsistency i.e. identifying a minimal set of axioms which make the ontology inconsistent.

In [79] and [56] the setting is extended to repairing ontologies connected by mappings. In this case, semantic defects may be introduced by integrating ontologies. Meilicke et al. [79] address the problem of finding and repairing incoherences caused by the automatic mapping process. The proposed debugging algorithm is based on conflict sets. The conflict sets extend the idea of diagnosis [84] where given a set of components $C O M P$ and a set of observations $O B S$, the task is to identify a subset of erroneous components so that the remaining components are consistent. In the case of distributed ontologies, a set of components COMP represents a set of mappings between ontologies and set of observations $O B S$ is a set of concept subsumptions in the ontologies. The proposed algorithm returns minimal conflict sets, i.e., conflict sets whose subsets are not conflict sets. Thus, repairing one component from the minimal conflict set would repair the conflict. The decision on which mappings should be removed from the minimal conflict steps is based on the confidence value of each mapping, if specified, or on the WordNet [9] distances between concepts participating in the mapping.

Ji et al. [56] propose a relevance-directed approach for diagnosing and 
repairing ontology networks. Their approach is based on computing MUPS between sub-ontologies and is adapted from the algorithm in [58] which is based on Reiter's hitting set algorithm [84]. The approach is relevancedirected as there are 3 strategies for computing MUPS: computing a single MUPS, computing some MUPS and some hitting sets and computing all MUPS and all hitting sets. The decision on which strategy to take is made by a user given an ontology and his/her purpose.

The work in [82] further characterizes the problem as mapping revision. The authors define a conflict-based mapping revision operator based on minimal conflict sets adapted from [79]. Using belief revision theory, the authors give an analysis for the logical properties of the revision algorithms.

Another approach for debugging mappings is proposed in [95]. In this work, the authors defined four types of defects in mappings, i.e. redundant mappings, imprecise mappings, inconsistent mappings and abnormal mappings. In addition, algorithms for detecting and diagnosing each type of defined defects were defined. The algorithms output warnings about detected defects as well as suggestions on how to debug them. The users have to manually execute the suggestions.

Work that deals with both modeling and semantic defects includes [27] where the authors propose an approach for detecting modeling and semantic defects within an ontology based on patterns and antipatterns. The proposed patterns and antipatterns contain both those patterns which have an impact on logical consequences as well as those which are supposed to improve the intended meaning of ontologies and their understandability. In addition to defining patterns and antipatterns, the authors also propose ways of solving the issues detected by the patterns and antipatterns. The work specifies a debugging strategy which defines the order in which a user should resolve detected patterns and antipatterns.

In [85] a method for detecting one of the antipatterns from [27] without a reasoner is provided. The antipatterns are detected by running a SPARQL query on a transformed ontology. A transformed ontology is acquired from the original ontology by applying transformation rules which aim at simplifying class definitions, harmonizing different implementation styles and simulating inferences thus avoiding the need for a reasoner.

Another work which considers both modeling and semantic defects is [69] where the authors provide a method for repairing wrong missing isa structure as well as wrong mappings in ontology networks which uses approaches for debugging semantic defects.

\subsection{Abductive reasoning in description logics}

In [38] four different abductive reasoning tasks are defined - concept abduction, ABox abduction, TBox abduction and knowledge base abduction.

Most existing approaches for description logic abduction focus on ABox and concept abduction and are mostly based on existing proof techniques 
such as semantic tableaux and resolution. Since the number of possible solutions can be infinite, the approaches introduce constraints on solutions.

In [46] the authors propose a semantic tableaux approach for ABox abduction in $\mathcal{A L C}$. In their approach, the authors perform instance checking on an abductive query. After extracting a full completion graph, the algorithm generates a set of concept assertions for each open branch which would close that branch. This is done by taking negations of concept assertions in open branches. The solutions to the abductive query are then acquired by running the minimal hitting set algorithm [84] on these sets. The algorithm is sound but not complete and the solutions are not guaranteed to be semantically minimal.

The work in [64] proposes a semantic-tableaux and a resolution based approach for ABox abduction. The proposed approaches are goal-oriented meaning that only actions which contribute to the solution are chosen in the proof procedures. This is done by using regular connection tableaux for the tableau-based approach or sets-of-support in the case of resolution proofs. Both approaches, however, require translation to first order logic (conjunctive normal form) and the solutions have to be translated back to description logic. The method is both complete and sound for consistent and semantically minimal solutions. Since the set of solutions can contain some inconsistent and non-minimal solutions additional checks are required to guarantee consistency and minimality.

A practical approach for ABox abduction, based on abductive logic programming, was proposed in [34]. This approach considers solutions over a finite set of concepts and roles called abducibles which is in contrast to the work in [64] where solutions can be formed with arbitrary concepts. As a result, the approach in [64] can return infinitely many solutions when dealing with cyclic definitions. The proposed approach uses existing abductive logic programming systems and therefore requires a transformation to a plain Datalog program. The solutions are consistent and minimal given a set of abducibles. In this case, allowed abducibles are atomic concepts or atomic roles. The approach does not guarantee completeness since the translation to a Datalog program is approximate and in some cases a solution would not be found. The authors extended their work in [35] by allowing arbitrary concepts and roles as abducibles, such as complex concepts, negated roles, etc. In [36] the authors further extend their previous work and deal with an ABox abduction problem where new individuals are allowed in solutions. For example, for abductive query Person(Tom) in the case where the ontology contains $\exists$ hasParent.Person $\sqsubseteq$ Person an intuitive minimal explanation is a set $\{$ hasParent $($ Tom,$u)$, Person $(u)\}$ where $u$ is a new individual. As a consequence of this extension there can be infinitely many minimal solutions. To deal with this, the authors reduce the number of explanations which need to be computed by considering only representative solutions, that is solutions which are not subsumed by other minimal solutions. The approach was compared to the approach in [34] in an experi- 
mental evaluation which has shown that computing representative solutions is more efficient than computing minimal solutions.

There are number of approaches which deal with the concept abduction problem as well as the conditionalised concept abduction problem where the idea is for concepts $C$ and $D$ and a TBox $T$ to identify a concept $H$ such that $C \sqcap H \sqsubseteq D$ holds in $T$ and $C \sqcap H \nsubseteq \perp$.

Colucci et al. [25] consider an approach for conditionalized concept abduction problem. The presented approach uses a variation of the semantic tableaux and two labelling functions were proposed. The two labelling functions $T()$ and $F()$ represent true and false formulas in a tableaux. The solutions are formed from concepts which have at least one constraint in $\mathrm{F}$ () of every open branch. This choice is non-deterministic and can be used to select solutions based on some criteria. The algorithm also contains a consistency check which implies that the produced solutions are always consistent.

Another approach for conditionalized concept abduction in $\mathcal{A L N}$ description logics was proposed in [29]. This approach operates on concepts in conjunctive normal form and is based on a modified version of structural subsumption algorithm from [22]. A structural subsumption algorithm is used to identify concepts $H$ which need to be conjoined with $C$ so that $C \sqcap H \sqsubseteq D$ holds. The proposed algorithm returns irreducible solutions, i.e. solutions which are minimal conjunctions.

The complexity of concept abduction in the $\mathcal{E} \mathcal{L}$ family of description logics been explored in [19]. The abduction problem discussed in the paper includes a set of possible hypotheses. The work focuses on three decision problems, i.e. existence (does a solution exist), relevance (does the hypothesis appear in some solution) and necessity (does the hypothesis appear in all solutions). The complexity of the decision problems is analysed in terms of a number of preference criteria, such as subset minimality, minimal cardinality and minimal weight. The analysis has shown that the complexity of concept abduction for the decision problems is equal to the complexity of abduction in propositional definite Horn theories in the case of $\mathcal{E} \mathcal{L}$ and $\mathcal{E} \mathcal{L}^{+}$. In the case of $\mathcal{E} \mathcal{L}^{++}$the complexity is the same as in the case of propositional Horn theories.

There has not been much work related to TBox abduction, which is the most relevant abduction problem for this thesis. The work in [53] proposes an automata-based approach to TBox abduction using abducibles. It is based on a reduction to the axiom pinpointing problem which is then solved with automata-based methods. Similar to the approach presented by Du et al. [34] the abductive problem also contains a set of abducibles which in this case represent a set of axioms that can appear in solutions.

A recent approach [47] considers the TBox abduction problem in $\mathcal{A L C}$ ontologies. The presented approach is based on tableaux reasoning where the solutions to the abductive query are acquired by generating a set of axioms for each open branch in the full completion graph such when added 
to the knowledge base, it would close the open branch. The axioms which close open branches are of the form $\mathrm{C} \sqsubseteq \mathrm{D}$ where $\mathrm{C}$ is of the form $A, \neg A$ or $\exists r . T$ and $\mathrm{D}$ is of the form $A, \neg A$ or $\forall r$. $\top$ where $A$ is an atomic concept and $r$ an atomic role. Similar to the approach in [46] the authors use the minimal hitting set algorithm [84] to extract a solution from these sets of axioms.

The authors have also shown that their approach for solving a TBox abduction problem can be applied to the generalized version of TBox abduction problem which considers multiple subsumption axioms in the abductive query. In this case for an abductive query $\left\{C_{1} \sqsubseteq D_{1}, \ldots, C_{n} \sqsubseteq D_{n}\right\}$ the satisfiability checker needs to be run on input $\left(C_{1} \sqcap \neg D_{1}\right) \sqcup \ldots \sqcup\left(C_{n} \sqcap \neg D_{n}\right)$.

Both the approach for the TBox abduction problem and the generalized version of TBox abduction problem require a post-processing step to test for semantic minimality, consistency and relevance. The approaches are sound and complete with respect to the allowed constructors for relations in the solution.

The presented approaches for description logic abduction mostly deal with relatively inexpressive ontologies, such as $\mathcal{E} \mathcal{L}$ and $\mathcal{A L C}$. However, the work [30] describes a type of conditionalised concept abduction called structural abduction which is applicable to $\mathcal{S H}$. In this case instead of finding concept $H$ such that $C \sqcap H \sqsubseteq D$ the presented algorithm first finds places within a description of $C$ where hypotheses variables can be added. Then solution is defined as substitution of a set of variables with concepts which make the new extended concept subsumed by $D$.

The computational complexity of the generalized TBox abduction problem in the $\mathcal{E} \mathcal{L}$ family of description logics was analysed in [96]. The analysis focused on three decision problems: existence (does a solution exist), relevance (does a set of axioms appear in some solution) and necessity (does a set of axioms appear in every solution). These problems relate to the problems discussed in [19] with a difference that our abduction problem does not include a set of hypotheses but an oracle function which returns true or false depending if an is-a relation is correct according to the domain or not. The computational complexity of these problems was explored with respect to the preference criteria presented in this thesis. The analysis has shown that skyline optimal solutions are the best choice in practice. 


\section{Chapter 7}

\section{Conclusion and Future Work}

The World Wide Web (WWW) is growing rapidly. However, data on the WWW has often only limited structure. This limits the opportunities for automation of queries which require the understanding of the meaning of the underlying data as well as queries which require data from multiple sources. As a consequence large amounts of useful data are not being used to their full potential.

A step towards the support for this kind of queries is the idea of the Semantic Web which represents an extension of the current WWW. The Semantic Web aims at structuring the data, thus adding meaning to the data which would allow automated agents to execute more sophisticated queries.

One of the key technologies in this endeavour are ontologies. Ontologies provide means for modeling a domain of interest i.e. establishing a vocabulary of the domain. Given this, ontologies can be used for data integration and as a basis of data interoperability.

Developing ontologies is not easy and it is often the case that resulting ontologies are incomplete. As a consequence semantically-enabled applications which use such ontologies might omit relevant and correct results. Therefore, there is a need for high quality ontologies.

In this thesis we have focused on completing the is-a structure in ontologies represented in description logics, more specifically the $\mathcal{E} \mathcal{L}$ family and $\mathcal{A L C}$. The $\mathcal{E} \mathcal{L}$ family of description logics is highly relevant for the representation of lightweight ontologies and some of the major biomedical ontologies are represented in $\mathcal{E} \mathcal{L}$. On the other hand, $\mathcal{A L C}$ is often considered a basic expressive description logic containing logical constructors such as disjunction, existential quantifier and universal quantifier which sets a foundation for more expressive description logics. Completing the is-a structure in ontologies consists of two phases, detection and repair. The de- 
tection phase is often does not detect all missing is-a relations as detection algorithms usually have very high precision but very low recall. Therefore, there exist more interesting approaches for repairing missing is-a structure than the naive approach where the detected missing is-a relations are just added to the ontology. In addition to repairing missing is-a structure, these more interesting approaches introduce new knowledge to the ontology which was not detected by the detection algorithm.

In relation to the objective To formalize the problem of repairing missing is-a structure in lightweight ontologies, we formalized the problem of completing the is-a structure in ontologies as a generalized version of the TBox abduction problem. In our formalization we consider the role of a domain expert as this type of defects requires domain knowledge to detect and resolve. In contrast to logic-based abduction where the idea is to identify as small solutions as possible, the goal of repairing missing is-a structure is to add more subsumptions to enrich the ontology. Therefore, our formalization also includes a preference criterion which emphasizes the informativeness i.e. the knowledge added to the ontology. This criterion, semantic maximality, prefers solutions which are the most informative, i.e. add the largest body of knowledge to the ontology.

We have analysed combinations of semantic maximality with a minimality preference which prefers non-redundant solutions (subset minimality) and identified three ways of combining them depending on what kind of priority is assigned to each preference. Analysis of these combinations has shown that even though solutions with maximal informativeness without redundancy are preferred, in practice it is not clear how to generate these solutions. Therefore, skyline optimal solutions are the next best thing. They represent non-redundant solutions at a certain level of informativeness. The idea is to reach as high level of informativeness as possible.

With respect to the objective To develop algorithms for repairing missing is-a structure in lightweight ontologies, we have developed algorithms for solving the GTAP in the $\mathcal{E} \mathcal{L}$ family and $\mathcal{A L C}$ description logics. The $\mathcal{E} \mathcal{L}$ algorithm relies on identifying logical patterns in normalized $\mathcal{E} \mathcal{L}$ ontologies for extracting solutions to GTAP. In this case, the reasoner is used as a black box and the logical patterns are applied on the result of the reasoning process. On the other hand, the $\mathcal{A L C}$ algorithm is more general and is based on the satisfiability checking using tableaux reasoning. This approach is a glass-box approach as the logical patterns used to identify solutions to the GTAP are applied directly on a completion graph of a tableaux reasoner. The algorithms are iterative and in every iteration, except for the last, a skyline optimal solution is acquired on a higher level of informativeness.

With regards to the objective To develop a system for repairing missing is-a structure in lightweight ontologies and analyse the usefulness of such system, we have implemented systems for completing $\mathcal{E} \mathcal{L}$ and $\mathcal{A L C}$ ontologies based on the algorithms. The analysis and the experiments have shown that our approach for completing the is-a structure in ontologies is useful as we 
have identified a number of new is-a relations in the experiments which were not detected by the detection algorithm. The iterative approach has also proven to be advantageous as in subsequent iterations new knowledge was identified and solutions to some missing is-a relations were refined.

\subsection{Future work}

There are a number of directions which are interesting for future work. They focus on improvements of the current approaches as well as extensions such as dealing with more expressive languages and wrong is-a relations. We list some of the interesting future work directions:

Improving scalability: As discussed, the algorithm for solving GTAP in $\mathcal{A L C}$ ontologies can not deal with larger more expressive ontologies. In these cases the completion graphs produced by running satisfiability checks on missing is-a relations contain many disjunctions. This would mean that there are many possible ways of closing open ABoxes. The solution extraction phase in our case is computationally demanding as when searching for solutions we explore all possible combinations for closure of open ABoxes (with respect to our heuristic of combining positive concept names with negated ones in open ABoxes). There are a number of approaches for restricting the search space and one approach was discussed in Section 5.4.1 where it was suggested that we only explore solutions to a certain level in the completion graph.

A more general approach is to investigate modular approaches for debugging ontologies. Here the idea is to run debugging algorithms on parts of ontologies which are relevant for a certain missing is-a relation (or a set of missing is-a relations) thus limiting the search space. There are a number of approaches for extracting ontology modules such as [42] and [31] which aim at identifying a fragment of an ontology which is relevant for some application thus supporting reuse as well as improving performance.

When it comes to computation time, this can be improved by parallelizing the computation of GTAP solutions to individual missing is-a relations. This is possible in our case as executions of the RepairSingleIs $A$ procedure are done independently of each other.

Integrating detection of missing is-a relations: So far we have only focused on the repairing phase. Our approach for repairing missing is-a relations can be considered as a detection method given that solutions to GTAP often contain new is-a relations which were not detected by the detection algorithm. In addition, our system could be run with a set of already derivable is-a relations as way of investigating and revising the existing is-a relations. However, these detection mecha- 
nisms explore only small parts of ontologies which are related to is-a relations under inspection.

A detection component would be beneficial for our approach as it would identify additional missing is-a relations and provide a starting point for the process of completing ontologies. There exist many approaches for detecting missing is-a relations in ontologies and some of them were described in Chapter 6.

Debugging wrong is-a relations: Our work focused only on missing is-a relations. Therefore, the next step would be to extend our approach to consider wrong is-a relations as well. As with missing is-a relations, debugging wrong is-a relations consists of two phases, detection and repair. In the repairing phase, the idea is to acquire justifications for a wrong is-a relation and try to remove or change relations in the ontology so that the wrong is-a relation is no longer derivable. When dealing with more expressive description logics containing logical constructs such as concept negation and the bottom concept, unsatisfiable concepts and inconsistencies can be a sign of wrong is-a relations in an ontology. Therefore, approaches for detecting and debugging semantic defects can be utilized, to a certain extent, when dealing with wrong is-a relations.

Therefore, a step towards dealing with wrong is-a relations would be to investigate the relation between semantic defects and wrong is-a relations in our setting. The results of this analysis can then be used for the formalization of the problem of debugging wrong is-a relations as well as for the development of algorithms for dealing with such defects.

More expressive representation languages: Another direction is to consider ontologies represented in more expressive description logics containing logical constructors such as cardinality restrictions and nominals. Given that the $\mathcal{A L C}$ algorithm is general and could be applicable to more expressive languages, the first step towards this goal would be to analyse our current approach for repairing missing missing is-a structure in $\mathcal{A L C}$ ontologies and identify what is the upper limit for expressiveness of ontologies that our approach can deal with.

Visualization techniques: The analysis of sizes of Source and Target sets in the evaluation of the $\mathcal{E} \mathcal{L}$ algorithm has shown that in the two experiments the sizes of Source and Target sets were manageable and the maximum number of concepts in Source and Targets sets was less than 400. However, the visualization can become cluttered even with this number of concepts which can be a problem for identifying is-a relations that need to be validated. Further, the visualization lacks support for presenting already validated relations as well as the consequences of adding some is-a relations. For example, there is no support 
for marking which other is-a relations are automatically validated to be correct.

The current visualization is not applicable for representation of larger and more expressive ontologies containing logical constructors such as quantifiers and disjunction. Dealing with this issue would be beneficial, as it would allow the user to check the current state of an ontology at any point of the completion process as well as get the bigger picture of relations between concepts. 



\section{Bibliography}

[1] BioPortal. http://bioportal.bioontology.org/. Accessed: 201408-20.

[2] Internet Live Stats. http://www. internetlivestats.com/. Accessed: 2014-10-13.

[3] MeSH: Medical Subject Headings. http://www.nlm.nih.gov/mesh/. Accessed: 2014-08-20.

[4] Netcraft Web Server Survey. http://news.netcraft.com/archives/ 2014/10/10/october-2014-web-server-survey.html. Accessed: 2014-10-13.

[5] PubMed. http://www.ncbi.nlm.nih.gov/pubmed. Accessed: 201408-20.

[6] SNOMED Clinical Terms. http://www.ihtsdo.org/snomed-ct/. Accessed: 2014-08-20.

[7] Uberon. http://www.uberon.org/. Accessed: 2014-08-20.

[8] UMLS Metathesaurus: Unified Medical Language System Metathesaurus. http://www.nlm.nih.gov/research/umls/knowledge_ sources/metathesaurus/. Accessed: 2014-08-20.

[9] WordNet. http://wordnet.princeton.edu/. Accessed: 2014-08-20.

[10] J. H. Alexander, M. J. Freiling, S. Shulman, J. Staley, S. Rehfuss, and S. Messick. Knowledge level engineering ontological analysis. In Proceedings of the 5th National Conference on Artificial Intelligence (AAAI), pages 963-968, 1986.

[11] P. Arnold and E. Rahm. Semantic enrichment of ontology mappings: a linguistic-based approach. In Proceedings of the 17th East-European Conference on Advances in Databases and Information Systems (AD$B I S)$, pages 42-55, 2013. 
[12] M. Ashburner, C. A. Ball, J. A. Blake, D. Botstein, H. Butler, J. M. Cherry, A. P. Davis, K. Dolinski, S. S. Dwight, J. T. Eppig, M. A. Harris, D. P. Hill, L. Issel-Tarver, A. Kasarskis, S. Lewis, J. C. Matese, J. E. Richardson, M. Ringwald, G. M. Rubin, and G. Sherlock. Gene Ontology: Tool for the Unification of Biology. Nature Genetics, 25(1):25-29, 2000 .

[13] F. Baader, S. Brandt, and C. Lutz. Pushing the $\mathcal{E} \mathcal{L}$ envelope. In Proceedings of the 19th International Joint Conference on Artificial Intelligence (IJCAI), pages 364-369, 2005.

[14] F. Baader, I. Horrocks, and U. Sattler. Description logics. In Handbook of knowledge representation, pages 135-179. 2008.

[15] F. Baader and W. Nutt. Basic description logics. In Description logic handbook, pages 43-95, 2003.

[16] F. Baader and U. Sattler. An overview of tableau algorithms for description logics. Studia Logica, 69:5-40, 2001.

[17] M. Bada and L. Hunter. Identification of OBO nonalignments and its implications for OBO enrichment. Bioinformatics, 24(12):1448-1455, 2008.

[18] T. Berners-Lee, J. Hendler, and O. Lassila. The Semantic Web. Scientific American, 284(5):28-37, 2001.

[19] M. Bienvenu. Complexity of abduction in the $\mathcal{E} \mathcal{L}$ family of lightweight description logics. In Proceedings of the 11th International Conference on Principles of Knowledge Representation and Reasoning (KR), pages 220-230, 2008.

[20] D. M. Blei, A. Y. Ng, and M. I. Jordan. Latent Dirichlet Allocation. Journal of Machine Learning Research, 3:993-1022, 2003.

[21] O. Bodenreider, T. F. Hayamizu, M. Ringwald, S. De Coronado, and S. Zhang. Of mice and men: Aligning mouse and human anatomies. In Proceedings of the 2005 AMIA Annual Symposium, pages 61-65, 2005.

[22] A. Borgida and P. F. Patel-Schneider. A semantics and complete algorithm for subsumption in the CLASSIC description logic. Journal of Artificial Intelligence Research, 1:277-308, 1994.

[23] P. Buitelaar, P. Cimiano, and B. Magnini. Ontology Learning from Text: Methods, Evaluation and Applications. 2005.

[24] P. Cimiano, A. Hotho, and S. Staab. Learning concept hierarchies from text corpora using formal concept analysis. Journal of Artifcial Intelligence Research, 24:305-339, 2005. 
[25] S. Colucci, T. Di Noia, E. Di Sciascio, F. M. Donini, and M. Mongiello. A uniform tableaux-based approach to concept abduction and contraction in $\mathcal{A L N}$. In Proceedings of the 17th International Workshop on Description Logics (DL), pages 158-167, 2004.

[26] O. Corcho, M. Fernández-López, and A. Gómez-Pérez. Ontological engineering: principles, methods, tools and languages. In Ontologies for software engineering and software technology, pages 1-48. 2006.

[27] O. Corcho, C. Roussey, L. M. Vilches, and I. Pérez. Pattern-based OWL ontology debugging guidelines. In Proceedings of the 1st Workshop on Ontology Patterns (WOP), pages 68-82, 2009.

[28] J. C. de Almeida Biolchini, P. G. Mian, A. C. C. Natali, T. U. Conte, and G. H. Travassos. Scientific research ontology to support systematic review in software engineering. Advanced Engineering Informatics, 21(2):133-151, 2007.

[29] T. Di Noia, E. Di Sciascio, and F. M. Donini. Semantic matchmaking as non-monotonic reasoning: A description logic approach. Journal of Artificial Intelligence Research, 29:269-307, 2007.

[30] F. M. Donini, S. Colucci, T. Di Noia, and E. Di Sciasco. A tableauxbased method for computing least common subsumers for expressive description logics. In Proceedings of the 21st International Joint Conference on Artificial Intelligence (IJCAI), pages 739-745, 2009.

[31] P. Doran, V. Tamma, and L. Iannone. Ontology module extraction for ontology reuse: an ontology engineering perspective. In Proceedings of the 16th ACM conference on Conference on information and knowledge management, pages 61-70, 2007.

[32] Z. Dragisic, P. Lambrix, and F. Wei-Kleiner. Completing the is-a structure of biomedical ontologies. In Proceedings of the 10th Conference on Data Integration in the Life Sciences (DILS), pages 66-80. 2014.

[33] Z. Dragisic, P. Lambrix, and F. Wei-Kleiner. A system for debugging missing is-a structure in $\mathcal{E} \mathcal{L}$ ontologies. In Proceedings of the $3 \mathrm{rd} \mathrm{In-}$ ternational Workshop on Debugging Ontologies and Ontology Mappings (WoDOOM), pages 51-58. 2014.

[34] J. Du, G. Qi, Y.-D. Shen, and J. Z. Pan. Towards practical ABox abduction in large OWL DL ontologies. In Proceedings of the 25th AAAI Conference on Artificial Intelligence, pages 1160-1165, 2011.

[35] J. Du, G. Qi, Y.-D. Shen, and J. Z. Pan. Towards practical ABox abduction in large description logic ontologies. International Journal on Semantic Web and Information Systems (IJSWIS), 8(2):1-33, 2012. 
[36] J. Du, K. Wang, and Y.-D. Shen. A tractable approach to ABox abduction over description logic ontologies. In Proceedings of the 28th AAAI Conference on Artificial Intelligence, pages 1034-1040, 2014.

[37] T. Eiter and G. Gottlob. The complexity of logic-based abduction. Journal of the ACM, 42(1):3-42, 1995.

[38] C. Elsenbroich, O. Kutz, and U. Sattler. A case for abductive reasoning over ontologies. In Proceedings of the 2nd International Workshop OWL: Experiences and Directions (OWLED), 2006.

[39] J. Euzenat and P. Shvaiko. Ontology matching. 2007.

[40] G. Flouris, D. Manakanatas, H. Kondylakis, D. Plexousakis, and G. Antoniou. Ontology Change: Classification and Survey. Knowledge Engineering Review, 23(2):117-152, 2008.

[41] B. Ganter and R. Wille. Formal concept analysis. 1999.

[42] B. Cuenca Grau, I. Horrocks, Y. Kazakov, and U. Sattler. Modular reuse of ontologies: Theory and practice. Journal of Artificial Intelligence Research, 31:273-318, 2008.

[43] T. R. Gruber. A translation approach to portable ontology specifications. Knowledge acquisition, 5(2):199-220, 1993.

[44] N. Guarino. Some ontological principles for designing upper level lexical resources. In Proceedings of the 1st International Conference on Language Resources and Evaluation (LREC), pages 527-534, 1998.

[45] P. Haase and L. Stojanovic. Consistent Evolution of OWL Ontologies. In Proceedings of the 2nd European Semantic Web Conference (ESWC), pages 182-197, 2005.

[46] K. Halland and K. Britz. Naive ABox abduction in $\mathcal{A L C}$ using a DL tableau. In Proceedings of the 25th International Workshop on Description Logics (DL), pages 443-453, 2012.

[47] K. Halland, K. Britz, and S. Klarman. TBox abduction in $\mathcal{A L C}$ using a DL tableau. In Proceedings of the 27th International Workshop on Description Logics (DL), pages 556-566, 2014.

[48] H. J. Happel and S. Seedorf. Applications of ontologies in software engineering. In Proceedings of the 2nd Workshop on Semantic Web Enabled Software Engineering (SWESE), pages 5-9, 2006.

[49] M. Hartung, J. Terwilliger, and E. Rahm. Recent advances in schema and ontology evolution. In Schema matching and mapping, pages 149190. 2011. 
[50] M. Hearst. Automatic acquisition of hyponyms from large text corpora. In Proceedings of the 14th International Conference on Computational Linguistics (COLING), pages 539-545, 1992.

[51] A. Herzog, N. Shahmehri, and C. Duma. An ontology of information security. International Journal of Information Security and Privacy (IJISP), 1(4):1-23, 2007.

[52] M. Horridge, B. Parsia, and U. Sattler. Extracting justifications from BioPortal ontologies. In Proceedings of the 11th International Semantic Web Conference (ISWC), pages 287-299, 2012.

[53] T. Hubauer, S. Lamparter, and M. Pirker. Automata-based abduction for tractable diagnosis. In Proceedings of the 23rd International Workshop on Description Logics (DL), pages 360-371, 2010.

[54] V. Ivanova and P. Lambrix. A unified approach for aligning taxonomies and debugging taxonomies and their alignments. In Proceedings of the 10th Extended Semantic Web Conference (ESWC), pages 1-15, 2013.

[55] V. Ivanova, J. Laurila Bergman, U. Hammerling, and P. Lambrix. Debugging taxonomies and their alignments: the ToxOntology - MeSH use case. In Proceedings of the 1st International Workshop on Debugging Ontologies and Ontology Mappings (WoDOOM), pages 25-36, 2012.

[56] Q. Ji, P. Haase, G. Qi, P. Hitzler, and S. Stadtmuller. RaDON - repair and diagnosis in ontology networks. In Proceedings of the 6th European Semantic Web Conference (ESWC), pages 863-867, 2009.

[57] A. Kalyanpur. Debugging and repair of OWL ontologies. PhD thesis, University of Maryland, 2006.

[58] A. Kalyanpur, B. Parsia, M. Horridge, and E. Sirin. Finding all justifications of OWL DL entailments. In Proceedings of the 6th International Semantic Web Conference (ISWC) and the 2nd Asian Semantic Web Conference (ASWC), pages 267-280. 2007.

[59] A. Kalyanpur, B. Parsia, E. Sirin, and B. Cuenca Grau. Repairing Unsatisfiable Concepts in OWL Ontologies. In Proceedings of the 3rd European Semantic Web Conference (ESWC), pages 170-184, 2006.

[60] A. Kalyanpur, B. Parsia, E. Sirin, and J. Hendler. Debugging Unsatisfiable Classes in OWL Ontologies. Journal of Web Semantics, 3(4):268293, 2006.

[61] Y. Kazakov, M. Krötzsch, and F. Simančík. Concurrent classification of $\mathcal{E} \mathcal{L}$ ontologies. In Proceedings of the 10th International Semantic Web Conference (ISWC), pages 305-320, 2011. 
[62] C. M. Keet. Detecting and revising flaws in OWL object property expressions. In Proceedings of the 18th Conference on Knowledge Engineering and Knowledge Management (EKAW), pages 252-266, 2012.

[63] A. Kim, J. Luo, and M. Kang. Security ontology for annotating resources. In Proceedings of the 4th International Conference on Ontologies, Databases, and Applications of Semantics (ODBASE), pages 1483-1499. 2005.

[64] S. Klarman, U. Endriss, and S. Schlobach. ABox abduction in the description logic $\mathcal{A L C}$. Journal of Automated Reasoning, 46:43-80, 2011.

[65] P. Lambrix. Ontologies in bioinformatics and systems biology. In Artificial Intelligence Methods and Tools for Systems Biology, pages 129-145. 2004 .

[66] P. Lambrix. Towards a semantic web for bioinformatics using ontologybased annotation. In Proceedings of 14th IEEE International Workshops on the Enabling Technologies: Infrastructure for Collaborative Enterprise, pages 3-7, 2005.

[67] P. Lambrix, Z. Dragisic, and V. Ivanova. Get my pizza right: Repairing missing is-a relations in $\mathcal{A L C}$ ontologies. In Proceedings of the 2nd Joint International Semantic Technology Conference (JIST), pages 1732,2012 .

[68] P. Lambrix and V. Ivanova. A unified approach for debugging is-a structure and mappings in networked taxonomies. Journal of Biomedical Semantics, 4:10, 2013.

[69] P. Lambrix and Q. Liu. Debugging is-a structure in networked taxonomies. In 4th International Workshop on Semantic Web Applications and Tools for Life Sciences (SWAT4LS), pages 58-65, 2011.

[70] P. Lambrix and Q. Liu. Debugging the missing is-a structure within taxonomies networked by partial reference alignments. Data $\&$ Knowledge Engineering, 86:179-205, 2013.

[71] P. Lambrix, Q. Liu, and H. Tan. Repairing the Missing is-a Structure of Ontologies. In Proceedings of the 4th Asian Semantic Web Conference $(A S W C)$, pages 76-90, 2009.

[72] P. Lambrix, F. Wei-Kleiner, Z. Dragisic, and V. Ivanova. Repairing missing is-a structure in ontologies is an abductive reasoning problem. In Proceedings of the 2nd International Workshop on Debugging Ontologies and Ontology Mappings (WoDOOM), pages 33-44, 2013.

[73] O. Lassila and D. McGuinness. The role of frame-based representation on the Semantic Web. Technical report, 2001. 
[74] D. B. Lenat. CYC: A large-scale investment in knowledge infrastructure. Communications of the ACM, 38(11):33-38, 1995.

[75] H. Lin and E. Sirin. Pellint - A performance lint tool for Pellet. In Proceedings of the 5th International Workshop OWL: Experiences and Directions (OWLED), 2008.

[76] C. Lutz. Complexity of terminiological reasoning revisited. In Proceedings of the 16th International Conference on Logic for Programming and Automated Reasoning (LPAR), pages 181-200, 1999.

[77] A. Maedche and S. Staab. Discovering conceptual relations from text. In Proceedings of the 14th European Conference on Artificial Intelligence (ECAI), pages 321-325, 2000.

[78] J. McCarthy. Circumscription-a form of non-monotonic reasoning. Artificial Intelligence, 13(1):27-39, 1980.

[79] C. Meilicke, H. Stuckenschmidt, and A. Tamilin. Repairing Ontology Mappings. In Proceedings of the 22th AAAI Conference on Artificial Intelligence, pages 1408-1413, 2007.

[80] R. Neches, R. E. Fikes, T. Finin, T. R. Gruber, R. Patil, T. Senator, and W. R. Swartout. Enabling technology for knowledge sharing. AI magazine, 12(3):36, 1991.

[81] N. F. Noy, N. H. Shah, P. L. Whetzel, B. Dai, M. Dorf, N. Griffith, C. Jonquet, D. L. Rubin, M. Storey, C. G. Chute, and M. A. Musen. Bioportal: ontologies and integrated data resources at the click of a mouse. Nucleic acids research, 37(suppl 2):W170-W173, 2009.

[82] G. Qi, Q. Ji, and P. Haase. A Conflict-Based Operator for Mapping Revision. In Proceedings of the 8th International Semantic Web Conference (ISWC), pages 521-536, 2009.

[83] J. C. Dos Reis, D. Dinh, C. Pruski, M. Da Silveira, and C. ReynaudDelaître. Mapping adaptation actions for the automatic reconciliation of dynamic ontologies. In Proceedings of the 22nd ACM International Conference on Information \& Knowledge Management (CIKM), pages 599-608, 2013.

[84] R. Reiter. A theory of diagnosis from first principles. Artificial Intelligence, 32(1):57-95, 1987.

[85] C. Roussey and A. Zamazal. Antipattern detection: How to debug an ontology without a reasoner. In Proceedings of the 2nd International Workshop on Debugging Ontologies and Ontology Mappings (WoDOOM), pages 45-56, 2013. 
[86] S. Schlobach. Debugging and Semantic Clarification by Pinpointing. In Proceedings of the 2nd European Semantic Web Conference (ESWC), pages 226-240, 2005.

[87] S. Schlobach and R. Cornet. Non-standard reasoning services for the debugging of description logic terminologies. In Proceedings of the 18th International Joint Conferences on Artificial Intelligence (IJCAI), volume 3, pages 355-362, 2003.

[88] M. Schmidt-Schauß and G. Smolka. Attributive concept descriptions with complements. Artificial Intelligence, 48(1):1-26, 1991.

[89] E. Sirin, B. Parsia, B. Cuenca Grau, A. Kalyanpur, and Y. Katz. Pellet: A practical OWL-DL reasoner. Web Semantics: science, services and agents on the World Wide Web, 5(2):51-53, 2007.

[90] V. Spiliopoulos, G. A. Vouros, and V. Karkaletsis. On the discovery of subsumption relations for the alignment of ontologies. Web Semantics: Science, Services and Agents on the World Wide Web, 8(1):69-88, 2010.

[91] A. Splendiani, A. Burger, A. Paschke, P. Romano, and S. M. Marshall. Biomedical semantics in the Semantic Web. Journal of biomedical semantics, 2(1):1-9, 2011.

[92] R. Stevens, C. A. Goble, and S. Bechhofer. Ontology-based knowledge representation for bioinformatics. Briefings in Bioinformatics, $1(4): 398-414,2000$.

[93] R. Studer, R. V. Benjamins, and D. Fensel. Knowledge engineering: principles and methods. Data \&3 knowledge engineering, 25(1):161-197, 1998.

[94] M. Uschold and M. Gruninger. Ontologies and semantics for seamless connectivity. ACM SIGMod Record, 33(4):58-64, 2004.

[95] P. Wang and B. Xu. Debugging ontology mappings: a static approach. Computing and Informatics, 27:21-36, 2008.

[96] F. Wei-Kleiner, Z. Dragisic, and P. Lambrix. Abduction framework for repairing incomplete $\mathcal{E} \mathcal{L}$ ontologies: Complexity results and algorithms. In Proceedings of the 28th AAAI Conference on Artificial Intelligence, pages 1120-1127, 2014.

[97] C. Welty. Ontology research. AI magazine, 24(3):11, 2003.

[98] E. Zavitsanos, G. Paliouras, G. A. Vouros, and S. Petridis. Discovering subsumption hierarchies of ontology concepts from text corpora. In Proceedings of the 2007 IEEE/WIC/ACM International Conference on Web Intelligence, pages 402-408, 2007. 


\section{Department of Computer and Information Science}

Linköpings universitet

\section{Licentiate Theses}

\section{Linköpings Studies in Science and Technology Faculty of Arts and Sciences}

No 17 Vojin Plavsic: Interleaved Processing of Non-Numerical Data Stored on a Cyclic Memory. (Available at: FOA, Box 1165, S-581 11 Linköping, Sweden. FOA Report B30062E)

No 28 Arne Jönsson, Mikael Patel: An Interactive Flowcharting Technique for Communicating and Realizing Algorithms, 1984.

No 29

No 48

No 52

No 60

No 71

No 72

No 73

No 74

No 104

No 108

No 111

No 113

No 118

No 126

No 127

No 139

No 140

No 146

No 150

No 165

No 166

No 174

No 177

No 181

No 184

No 187

No 189

No 196

No 197

No 203

No 212

No 230

No 237

No 250

No 253

No 260

No 283

No 298

No 318

No 319

No 326

No 328

No 333

No 335

No 348

No 352
Johnny Eckerland: Retargeting of an Incremental Code Generator, 1984.

Henrik Nordin: On the Use of Typical Cases for Knowledge-Based Consultation and Teaching, 1985.

Zebo Peng: Steps Towards the Formalization of Designing VLSI Systems, 1985.

Johan Fagerström: Simulation and Evaluation of Architecture based on Asynchronous Processes, 1985.

Jalal Maleki: ICONStraint, A Dependency Directed Constraint Maintenance System, 1987.

Tony Larsson: On the Specification and Verification of VLSI Systems, 1986.

Ola Strömfors: A Structure Editor for Documents and Programs, 1986.

Christos Levcopoulos: New Results about the Approximation Behavior of the Greedy Triangulation, 1986.

Shamsul I. Chowdhury: Statistical Expert Systems - a Special Application Area for Knowledge-Based Computer Methodology, 1987.

Rober Bilos: Incremental Scanning and Token-Based Editing, 1987.

Hans Block: SPORT-SORT Sorting Algorithms and Sport Tournaments, 1987.

Ralph Rönnquist: Network and Lattice Based Approaches to the Representation of Knowledge, 1987.

Mariam Kamkar, Nahid Shahmehri: Affect-Chaining in Program Flow Analysis Applied to Queries of Programs, 1987.

Dan Strömberg: Transfer and Distribution of Application Programs, 1987.

Kristian Sandahl: Case Studies in Knowledge Acquisition, Migration and User Acceptance of Expert Systems, 1987.

Christer Bäckström: Reasoning about Interdependent Actions, 1988.

Mats Wirén: On Control Strategies and Incrementality in Unification-Based Chart Parsing, 1988.

Johan Hultman: A Software System for Defining and Controlling Actions in a Mechanical System, 1988.

Tim Hansen: Diagnosing Faults using Knowledge about Malfunctioning Behavior, 1988.

Jonas Löwgren: Supporting Design and Management of Expert System User Interfaces, 1989.

Ola Petersson: On Adaptive Sorting in Sequential and Parallel Models, 1989.

Yngve Larsson: Dynamic Configuration in a Distributed Environment, 1989.

Peter Åberg: Design of a Multiple View Presentation and Interaction Manager, 1989.

Henrik Eriksson: A Study in Domain-Oriented Tool Support for Knowledge Acquisition, 1989.

Ivan Rankin: The Deep Generation of Text in Expert Critiquing Systems, 1989.

Simin Nadjm-Tehrani: Contributions to the Declarative Approach to Debugging Prolog Programs, 1989.

Magnus Merkel: Temporal Information in Natural Language, 1989.

Ulf Nilsson: A Systematic Approach to Abstract Interpretation of Logic Programs, 1989.

Staffan Bonnier: Horn Clause Logic with External Procedures: Towards a Theoretical Framework, 1989.

Christer Hansson: A Prototype System for Logical Reasoning about Time and Action, 1990.

Björn Fjellborg: An Approach to Extraction of Pipeline Structures for VLSI High-Level Synthesis, 1990.

Patrick Doherty: A Three-Valued Approach to Non-Monotonic Reasoning, 1990.

Tomas Sokolnicki: Coaching Partial Plans: An Approach to Knowledge-Based Tutoring, 1990.

Lars Strömberg: Postmortem Debugging of Distributed Systems, 1990.

Torbjörn Näslund: SLDFA-Resolution - Computing Answers for Negative Queries, 1990.

Peter D. Holmes: Using Connectivity Graphs to Support Map-Related Reasoning, 1991.

Olof Johansson: Improving Implementation of Graphical User Interfaces for Object-Oriented Knowledge- Bases, 1991.

Rolf G Larsson: Aktivitetsbaserad kalkylering i ett nytt ekonomisystem, 1991.

Lena Srömbäck: Studies in Extended Unification-Based Formalism for Linguistic Description: An Algorithm for Feature Structures with Disjunction and a Proposal for Flexible Systems, 1992.

Mikael Pettersson: DML-A Language and System for the Generation of Efficient Compilers from Denotational Specification, 1992.

Andreas Kågedal: Logic Programming with External Procedures: an Implementation, 1992.

Patrick Lambrix: Aspects of Version Management of Composite Objects, 1992.

Xinli Gu: Testability Analysis and Improvement in High-Level Synthesis Systems, 1992.

Torbjörn Näslund: On the Role of Evaluations in Iterative Development of Managerial Support Systems, 1992.

Ulf Cederling: Industrial Software Development - a Case Study, 1992.

Magnus Morin: Predictable Cyclic Computations in Autonomous Systems: A Computational Model and Implementation, 1992.

Mehran Noghabai: Evaluation of Strategic Investments in Information Technology, 1993.

Mats Larsson: A Transformational Approach to Formal Digital System Design, 1993. 
Johan Ringström: Compiler Generation for Parallel Languages from Denotational Specifications, 1993.

Michael Jansson: Propagation of Change in an Intelligent Information System, 1993.

Jonni Harrius: An Architecture and a Knowledge Representation Model for Expert Critiquing Systems, 1993.

Per Österling: Symbolic Modelling of the Dynamic Environments of Autonomous Agents, 1993.

Johan Boye: Dependency-based Groudness Analysis of Functional Logic Programs, 1993.

Lars Degerstedt: Tabulated Resolution for Well Founded Semantics, 1993.

Anna Moberg: Satellitkontor - en studie av kommunikationsmönster vid arbete på distans, 1993.

Peter Carlsson: Separation av företagsledning och finansiering - fallstudier av företagsledarutköp ur ett agentteoretiskt perspektiv, 1994.

Camilla Sjöström: Revision och lagreglering - ett historiskt perspektiv, 1994.

Cecilia Sjöberg: Voices in Design: Argumentation in Participatory Development, 1994.

Lars Viklund: Contributions to a High-level Programming Environment for a Scientific Computing, 1994.

Peter Loborg: Error Recovery Support in Manufacturing Control Systems, 1994.

Owen Eriksson: Informationssystem med verksamhetskvalitet - utvärdering baserat på ett verksamhetsinriktat och samskapande perspektiv, 1994.

Karin Pettersson: Informationssystemstrukturering, ansvarsfördelning och användarinflytande - En komparativ studie med utgångspunkt i två informationssystemstrategier, 1994.

Lars Poignant: Informationsteknologi och företagsetablering - Effekter på produktivitet och region, 1994.

Gustav Fahl: Object Views of Relational Data in Multidatabase Systems, 1994.

Henrik Nilsson: A Declarative Approach to Debugging for Lazy Functional Languages, 1994.

Jonas Lind: Creditor - Firm Relations: an Interdisciplinary Analysis, 1994.

Martin Sköld: Active Rules based on Object Relational Queries - Efficient Change Monitoring Techniques, 1994.

Pär Carlshamre: A Collaborative Approach to Usability Engineering: Technical Communicators and System Developers in Usability-Oriented Systems Development, 1994.

Stefan Cronholm: Varför CASE-verktyg i systemutveckling? - En motiv- och konsekvensstudie avseende arbetssätt och arbetsformer, 1994.

Mikael Lindvall: A Study of Traceability in Object-Oriented Systems Development, 1994.

Fredrik Nilsson: Strategi och ekonomisk styrning - En studie av Sandviks förvärv av Bahco Verktyg, 1994.

Hans Olsén: Collage Induction: Proving Properties of Logic Programs by Program Synthesis, 1994.

Lars Karlsson: Specification and Synthesis of Plans Using the Features and Fluents Framework, 1995.

Ulf Söderman: On Conceptual Modelling of Mode Switching Systems, 1995.

Choong-ho Yi: Reasoning about Concurrent Actions in the Trajectory Semantics, 1995.

Bo Lagerström: Successiv resultatavräkning av pågående arbeten. - Fallstudier i tre byggföretag, 1995.

Peter Jonsson: Complexity of State-Variable Planning under Structural Restrictions, 1995.

Anders Avdic: Arbetsintegrerad systemutveckling med kalkylprogram, 1995.

Eva L Ragnemalm: Towards Student Modelling through Collaborative Dialogue with a Learning Companion, 1995.

Eva Toller: Contributions to Parallel Multiparadigm Languages: Combining Object-Oriented and Rule-Based Programming, 1995.

Erik Stoy: A Petri Net Based Unified Representation for Hardware/Software Co-Design, 1995.

Johan Herber: Environment Support for Building Structured Mathematical Models, 1995.

Stefan Svenberg: Structure-Driven Derivation of Inter-Lingual Functor-Argument Trees for Multi-Lingual Generation, 1995.

Hee-Cheol Kim: Prediction and Postdiction under Uncertainty, 1995.

Dan Fristedt: Metoder i användning - mot förbättring av systemutveckling genom situationell metodkunskap och metodanalys, 1995.

Malin Bergvall: Systemförvaltning i praktiken - en kvalitativ studie avseende centrala begrepp, aktiviteter och ansvarsroller, 1995.

Joachim Karlsson: Towards a Strategy for Software Requirements Selection, 1995.

Jakob Axelsson: Schedulability-Driven Partitioning of Heterogeneous Real-Time Systems, 1995.

Göran Forslund: Toward Cooperative Advice-Giving Systems: The Expert Systems Experience, 1995.

Jörgen Andersson: Bilder av småföretagares ekonomistyrning, 1995.

Staffan Flodin: Efficient Management of Object-Oriented Queries with Late Binding, 1996.

Vadim Engelson: An Approach to Automatic Construction of Graphical User Interfaces for Applications in Scientific Computing, 1996.

Magnus Werner : Multidatabase Integration using Polymorphic Queries and Views, 1996.

Mikael Lind: Affärsprocessinriktad förändringsanalys - utveckling och tillämpning av synsätt och metod, 1996.

Jonas Hallberg: High-Level Synthesis under Local Timing Constraints, 1996.

Kristina Larsen: Förutsättningar och begränsningar för arbete på distans - erfarenheter från fyra svenska företag. 1996.

Mikael Johansson: Quality Functions for Requirements Engineering Methods, 1996.

Patrik Nordling: The Simulation of Rolling Bearing Dynamics on Parallel Computers, 1996.

Anders Ekman: Exploration of Polygonal Environments, 1996.

Niclas Andersson: Compilation of Mathematical Models to Parallel Code, 1996. 
Johan Jenvald: Simulation and Data Collection in Battle Training, 1996.

Niclas Ohlsson: Software Quality Engineering by Early Identification of Fault-Prone Modules, 1996.

Mikael Ericsson: Commenting Systems as Design Support-A Wizard-of-Oz Study, 1996.

Jörgen Lindström: Chefers användning av kommunikationsteknik, 1996.

Esa Falkenroth: Data Management in Control Applications - A Proposal Based on Active Database Systems, 1996.

Niclas Wahllöf: A Default Extension to Description Logics and its Applications, 1996.

Annika Larsson: Ekonomisk Styrning och Organisatorisk Passion - ett interaktivt perspektiv, 1997.

Ling Lin: A Value-based Indexing Technique for Time Sequences, 1997.

Rego Granlund: ${ }^{3}$ Fire - A Microworld Supporting Emergency Management Training, 1997.

Peter Ingels: A Robust Text Processing Technique Applied to Lexical Error Recovery, 1997.

Per-Arne Persson: Toward a Grounded Theory for Support of Command and Control in Military Coalitions, 1997. Jonas S Karlsson: A Scalable Data Structure for a Parallel Data Server, 1997.

Carita Åbom: Videomötesteknik i olika affärssituationer - möjligheter och hinder, 1997.

Tommy Wedlund: Att skapa en företagsanpassad systemutvecklingsmodell - genom rekonstruktion, värdering och vidareutveckling i T50-bolag inom ABB, 1997.

Silvia Coradeschi: A Decision-Mechanism for Reactive and Coordinated Agents, 1997.

Jan Ollinen: Det flexibla kontorets utveckling på Digital - Ett stöd för multiflex? 1997.

David Byers: Towards Estimating Software Testability Using Static Analysis, 1997.

Fredrik Eklund: Declarative Error Diagnosis of GAPLog Programs, 1997.

Gunilla Ivefors: Krigsspel och Informationsteknik inför en oförutsägbar framtid, 1997.

Jens-Olof Lindh: Analysing Traffic Safety from a Case-Based Reasoning Perspective, 1997

Jukka Mäki-Turja:. Smalltalk - a suitable Real-Time Language, 1997.

Juha Takkinen: CAFE: Towards a Conceptual Model for Information Management in Electronic Mail, 1997.

Man Lin: Formal Analysis of Reactive Rule-based Programs, 1997.

Mats Gustafsson: Bringing Role-Based Access Control to Distributed Systems, 1997.

Boris Karlsson: Metodanalys för förståelse och utveckling av systemutvecklingsverksamhet. Analys och värdering av systemutvecklingsmodeller och dess användning, 1997.

Marcus Bjäreland: Two Aspects of Automating Logics of Action and Change - Regression and Tractability, 1998.

Jan Håkegård: Hierarchical Test Architecture and Board-Level Test Controller Synthesis, 1998.

Per-Ove Zetterlund: Normering av svensk redovisning - En studie av tillkomsten av Redovisningsrådets rekommendation om koncernredovisning (RR01:91), 1998.

Jimmy Tjäder: Projektledaren \& planen - en studie av projektledning i tre installations- och systemutvecklingsprojekt, 1998.

Ulf Melin: Informationssystem vid ökad affärs- och processorientering - egenskaper, strategier och utveckling, 1998.

Tim Heyer: COMPASS: Introduction of Formal Methods in Code Development and Inspection, 1998.

Patrik Hägglund: Programming Languages for Computer Algebra, 1998.

Marie-Therese Christiansson: Inter-organisatorisk verksamhetsutveckling - metoder som stöd vid utveckling av partnerskap och informationssystem, 1998.

Christina Wennestam: Information om immateriella resurser. Investeringar i forskning och utveckling samt i personal inom skogsindustrin, 1998.

Joakim Gustafsson: Extending Temporal Action Logic for Ramification and Concurrency, 1998.

Henrik André-Jönsson: Indexing time-series data using text indexing methods, 1999.

Erik Larsson: High-Level Testability Analysis and Enhancement Techniques, 1998.

Carl-Johan Westin: Informationsförsörjning: en fråga om ansvar - aktiviteter och uppdrag i fem stora svenska organisationers operativa informationsförsörjning, 1998.

Åse Jansson: Miljöhänsyn - en del i företags styrning, 1998.

Thomas Padron-McCarthy: Performance-Polymorphic Declarative Queries, 1998.

Anders Bäckström: Värdeskapande kreditgivning - Kreditriskhantering ur ett agentteoretiskt perspektiv, 1998.

Ulf Seigerroth: Integration av förändringsmetoder - en modell för välgrundad metodintegration, 1999.

Fredrik Öberg: Object-Oriented Frameworks - A New Strategy for Case Tool Development, 1998.

Jonas Mellin: Predictable Event Monitoring, 1998.

Joakim Eriksson: Specifying and Managing Rules in an Active Real-Time Database System, 1998.

Bengt E W Andersson: Samverkande informationssystem mellan aktörer i offentliga åtaganden - En teori om aktörsarenor i samverkan om utbyte av information, 1998.

Pawel Pietrzak: Static Incorrectness Diagnosis of CLP (FD), 1999.

Tobias Ritzau: Real-Time Reference Counting in RT-Java, 1999.

Anders Ferntoft: Elektronisk affärskommunikation - kontaktkostnader och kontaktprocesser mellan kunder och leverantörer på producentmarknader, 1999.

Jo Skåmedal: Arbete på distans och arbetsformens påverkan på resor och resmönster, 1999.

Johan Alvehus: Mötets metaforer. En studie av berättelser om möten, 1999. 
Magnus Lindahl: Bankens villkor i låneavtal vid kreditgivning till högt belånade företagsförvärv: En studie ur ett agentteoretiskt perspektiv, 2000.

No 766 Martin V. Howard: Designing dynamic visualizations of temporal data, 1999.

Charlotte Björkegren: Learning for the next project - Bearers and barriers in knowledge transfer within an organisation, 1999.

No 788 Håkan Nilsson: Informationsteknik som drivkraft i granskningsprocessen - En studie av fyra revisionsbyråer, 2000 .

No $790 \quad$ Erik Berglund: Use-Oriented Documentation in Software Development, 1999.

No 791

Klas Gäre: Verksamhetsförändringar i samband med IS-införande, 1999.

No 800

Anders Subotic: Software Quality Inspection, 1999.

No 807

Svein Bergum: Managerial communication in telework, 2000.

No 809

Flavius Gruian: Energy-Aware Design of Digital Systems, 2000.

FiF-a 32

Karin Hedström: Kunskapsanvändning och kunskapsutveckling hos verksamhetskonsulter - Erfarenheter från ett FOU-samarbete, 2000.

No 808 Linda Askenäs: Affärssystemet - En studie om teknikens aktiva och passiva roll i en organisation, 2000.

No 820

No 823

No 832

FiF-a 34

Jean Paul Meynard: Control of industrial robots through high-level task programming, 2000.

Lars Hult: Publika Gränsytor - ett designexempel, 2000.

Paul Pop: Scheduling and Communication Synthesis for Distributed Real-Time Systems, 2000.

No 842

Göran Hultgren: Nätverksinriktad Förändringsanalys - perspektiv och metoder som stöd för förståelse och utveckling av affärsrelationer och informationssystem, 2000.

No 844

FiF-a 37

FiF-a 40

FiF-a 41

No. 854

Magnus Kald: The role of management control systems in strategic business units, 2000.

No 863

No 881

No 882

Mikael Cäker: Vad kostar kunden? Modeller för intern redovisning, 2000.

Ewa Braf: Organisationers kunskapsverksamheter - en kritisk studie av "knowledge management", 2000.

Henrik Lindberg: Webbaserade affärsprocesser - Möjligheter och begränsningar, 2000.

Benneth Christiansson: Att komponentbasera informationssystem - Vad säger teori och praktik?, 2000.

Ola Pettersson: Deliberation in a Mobile Robot, 2000.

Dan Lawesson: Towards Behavioral Model Fault Isolation for Object Oriented Control Systems, 2000.

Johan Moe: Execution Tracing of Large Distributed Systems, 2001.

Yuxiao Zhao: XML-based Frameworks for Internet Commerce and an Implementation of B2B e-procurement, 2001.

No 890

FiF-a 47

Annika Flycht-Eriksson: Domain Knowledge Management in Information-providing Dialogue systems, 2001.

No 894

No 906

No 917

No 916

Per-Arne Segerkvist: Webbaserade imaginära organisationers samverkansformer: Informationssystemarkitektur och aktörssamverkan som förutsättningar för affärsprocesser, 2001.

Stefan Svarén: Styrning av investeringar i divisionaliserade företag - Ett koncernperspektiv, 2001.

Lin Han: Secure and Scalable E-Service Software Delivery, 2001.

Emma Hansson: Optionsprogram för anställda - en studie av svenska börsföretag, 2001.

Susanne Odar: IT som stöd för strategiska beslut, en studie av datorimplementerade modeller av verksamhet som stöd för beslut om anskaffning av JAS 1982, 2002.

FiF-a-49 Stefan Holgersson: IT-system och filtrering av verksamhetskunskap - kvalitetsproblem vid analyser och beslutsfattande som bygger på uppgifter hämtade från polisens IT-system, 2001.

FiF-a-51 Per Oscarsson: Informationssäkerhet i verksamheter - begrepp och modeller som stöd för förståelse av informationssäkerhet och dess hantering, 2001.

No 919 Luis Alejandro Cortes: A Petri Net Based Modeling and Verification Technique for Real-Time Embedded Systems, 2001.

No 915 Niklas Sandell: Redovisning i skuggan av en bankkris - Värdering av fastigheter. 2001.

No 931 Fredrik Elg: Ett dynamiskt perspektiv på individuella skillnader av heuristisk kompetens, intelligens, mentala modeller, mål och konfidens i kontroll av mikrovärlden Moro, 2002.

No 933 Peter Aronsson: Automatic Parallelization of Simulation Code from Equation Based Simulation Languages, 2002.

No 938

No 942

No 956

FiF-a 58

No 964

No 973

No 958

Bourhane Kadmiry: Fuzzy Control of Unmanned Helicopter, 2002.

Patrik Haslum: Prediction as a Knowledge Representation Problem: A Case Study in Model Design, 2002.

Robert Sevenius: On the instruments of governance - A law \& economics study of capital instruments in limited liability companies, 2002.

Johan Petersson: Lokala elektroniska marknadsplatser - informationssystem för platsbundna affärer, 2002.

Peter Bunus: Debugging and Structural Analysis of Declarative Equation-Based Languages, 2002.

Gert Jervan: High-Level Test Generation and Built-In Self-Test Techniques for Digital Systems, 2002.

Fredrika Berglund: Management Control and Strategy - a Case Study of Pharmaceutical Drug Development, 2002.

FiF-a 61 Fredrik Karlsson: Meta-Method for Method Configuration - A Rational Unified Process Case, 2002.

No 985

No 982

No 989

No 990

Sorin Manolache: Schedulability Analysis of Real-Time Systems with Stochastic Task Execution Times, 2002.

Diana Szentiványi: Performance and Availability Trade-offs in Fault-Tolerant Middleware, 2002.

Iakov Nakhimovski: Modeling and Simulation of Contacting Flexible Bodies in Multibody Systems, 2002.

Levon Saldamli: PDEModelica - Towards a High-Level Language for Modeling with Partial Differential Equations, 2002.

No 991 Almut Herzog: Secure Execution Environment for Java Electronic Services, 2002. 
Jon Edvardsson: Contributions to Program- and Specification-based Test Data Generation, 2002.

Anders Arpteg: Adaptive Semi-structured Information Extraction, 2002.

Andrzej Bednarski: A Dynamic Programming Approach to Optimal Retargetable Code Generation for Irregular Architectures, 2002.

Mattias Arvola: Good to use! : Use quality of multi-user applications in the home, 2003.

Lennart Ljung: Utveckling av en projektivitetsmodell - om organisationers förmåga att tillämpa projektarbetsformen, 2003.

Pernilla Qvarfordt: User experience of spoken feedback in multimodal interaction, 2003.

Alexander Siemers: Visualization of Dynamic Multibody Simulation With Special Reference to Contacts, 2003.

Jens Gustavsson: Towards Unanticipated Runtime Software Evolution, 2003.

Calin Curescu: Adaptive QoS-aware Resource Allocation for Wireless Networks, 2003.

Anna Andersson: Management Information Systems in Process-oriented Healthcare Organisations, 2003.

Björn Johansson: Feedforward Control in Dynamic Situations, 2003.

Traian Pop: Scheduling and Optimisation of Heterogeneous Time/Event-Triggered Distributed Embedded Systems, 2003.

Britt-Marie Johansson: Kundkommunikation på distans - en studie om kommunikationsmediets betydelse i affärstransaktioner, 2003.

Aleksandra Tešanovic: Towards Aspectual Component-Based Real-Time System Development, 2003.

Arja Vainio-Larsson: Designing for Use in a Future Context - Five Case Studies in Retrospect, 2003.

Peter Nilsson: Svenska bankers redovisningsval vid reservering för befarade kreditförluster - En studie vid införandet av nya redovisningsregler, 2003.

Fredrik Ericsson: Information Technology for Learning and Acquiring of Work Knowledge, 2003.

Marcus Comstedt: Towards Fine-Grained Binary Composition through Link Time Weaving, 2003.

Åsa Hedenskog: Increasing the Automation of Radio Network Control, 2003.

Claudiu Duma: Security and Efficiency Tradeoffs in Multicast Group Key Management, 2003.

Emma Eliason: Effektanalys av IT-systems handlingsutrymme, 2003.

Carl Cederberg: Experiments in Indirect Fault Injection with Open Source and Industrial Software, 2003.

Daniel Karlsson: Towards Formal Verification in a Component-based Reuse Methodology, 2003.

Anders Hjalmarsson: Att etablera och vidmakthålla förbättringsverksamhet - behovet av koordination och interaktion vid förändring av systemutvecklingsverksamheter, 2004.

Pontus Johansson: Design and Development of Recommender Dialogue Systems, 2004.

Charlotte Stoltz: Calling for Call Centres - A Study of Call Centre Locations in a Swedish Rural Region, 2004.

Björn Johansson: Deciding on Using Application Service Provision in SMEs, 2004.

Genevieve Gorrell: Language Modelling and Error Handling in Spoken Dialogue Systems, 2004.

Ulf Johansson: Rule Extraction - the Key to Accurate and Comprehensible Data Mining Models, 2004.

Sonia Sangari: Computational Models of Some Communicative Head Movements, 2004.

Hans Nässla: Intra-Family Information Flow and Prospects for Communication Systems, 2004.

Henrik Sällberg: On the value of customer loyalty programs - A study of point programs and switching costs, 2004.

Ulf Larsson: Designarbete i dialog - karaktärisering av interaktionen mellan användare och utvecklare i en systemutvecklingsprocess, 2004.

Andreas Borg: Contribution to Management and Validation of Non-Functional Requirements, 2004.

Per-Ola Kristensson: Large Vocabulary Shorthand Writing on Stylus Keyboard, 2004.

Pär-Anders Albinsson: Interacting with Command and Control Systems: Tools for Operators and Designers, 2004.

Ioan Chisalita: Safety-Oriented Communication in Mobile Networks for Vehicles, 2004.

Thomas Gustafsson: Maintaining Data Consistency in Embedded Databases for Vehicular Systems, 2004.

Vaida Jakoniené: A Study in Integrating Multiple Biological Data Sources, 2005.

Abdil Rashid Mohamed: High-Level Techniques for Built-In Self-Test Resources Optimization, 2005.

Adrian Pop: Contributions to Meta-Modeling Tools and Methods, 2005.

Fidel Vascós Palacios: On the information exchange between physicians and social insurance officers in the sick leave process: an Activity Theoretical perspective, 2005.

Jenny Lagsten: Verksamhetsutvecklande utvärdering i informationssystemprojekt, 2005.

Emma Larsdotter Nilsson: Modeling, Simulation, and Visualization of Metabolic Pathways Using Modelica, 2005.

Christina Keller: Virtual Learning Environments in higher education. A study of students' acceptance of educational technology, 2005.

Cécile Åberg: Integration of organizational workflows and the Semantic Web, 2005.

Anders Forsman: Standardisering som grund för informationssamverkan och IT-tjänster - En fallstudie baserad på trafikinformationstjänsten RDS-TMC, 2005.

Yu-Hsing Huang: A systemic traffic accident model, 2005.

Jan Olausson: Att modellera uppdrag - grunder för förståelse av processinriktade informationssystem i transaktionsintensiva verksamheter, 2005.

Petter Ahlström: Affärsstrategier för seniorbostadsmarknaden, 2005.

Mathias Cöster: Beyond IT and Productivity - How Digitization Transformed the Graphic Industry, 2005.

Åsa Horzella: Beyond IT and Productivity - Effects of Digitized Information Flows in Grocery Distribution, 2005.

Maria Kollberg: Beyond IT and Productivity - Effects of Digitized Information Flows in the Logging Industry, 2005.

David Dinka: Role and Identity - Experience of technology in professional settings, 2005. 
Andreas Hansson: Increasing the Storage Capacity of Recursive Auto-associative Memory by Segmenting Data, 2005.

No 1192

No 1194

No 1204

No 1206

No 1207

No 1209

No 1225

No 1228

No 1229

No 1231

No 1233

No 1244

No 1248

No 1263

FiF-a 90

No 1272

No 1277

No 1283

FiF-a 91

No 1286

No 1293

No 1302

No 1303

No 1305

No 1306

No 1307

No 1309

No 1312

No 1313

No 1317

No 1320

No 1323

No 1329

No 1331

No 1332

No 1333

No 1337

No 1339

No 1351

No 1353

No 1356

No 1359

No 1361

No 1363

No 1371

No 1373

No 1381

No 1386

No 1387

No 1392

No 1393

No 1401

No 1410

No 1421

No 1427

No 1450

No 1459

No 1466

Nicklas Bergfeldt: Towards Detached Communication for Robot Cooperation, 2005.

Dennis Maciuszek: Towards Dependable Virtual Companions for Later Life, 2005.

Beatrice Alenljung: Decision-making in the Requirements Engineering Process: A Human-centered Approach, 2005 .

Anders Larsson: System-on-Chip Test Scheduling and Test Infrastructure Design, 2005.

John Wilander: Policy and Implementation Assurance for Software Security, 2005.

Andreas Käll: Översättningar av en managementmodell - En studie av införandet av Balanced Scorecard i ett landsting, 2005.

He Tan: Aligning and Merging Biomedical Ontologies, 2006.

Artur Wilk: Descriptive Types for XML Query Language Xcerpt, 2006.

Per Olof Pettersson: Sampling-based Path Planning for an Autonomous Helicopter, 2006.

Kalle Burbeck: Adaptive Real-time Anomaly Detection for Safeguarding Critical Networks, 2006.

Daniela Mihailescu: Implementation Methodology in Action: A Study of an Enterprise Systems Implementation Methodology, 2006.

Jörgen Skågeby: Public and Non-public gifting on the Internet, 2006.

Karolina Eliasson: The Use of Case-Based Reasoning in a Human-Robot Dialog System, 2006.

Misook Park-Westman: Managing Competence Development Programs in a Cross-Cultural Organisation - What are the Barriers and Enablers, 2006.

Amra Halilovic: Ett praktikperspektiv på hantering av mjukvarukomponenter, 2006.

Raquel Flodström: A Framework for the Strategic Management of Information Technology, 2006.

Viacheslav Izosimov: Scheduling and Optimization of Fault-Tolerant Embedded Systems, 2006.

Håkan Hasewinkel: A Blueprint for Using Commercial Games off the Shelf in Defence Training, Education and Research Simulations, 2006.

Hanna Broberg: Verksamhetsanpassade IT-stöd - Designteori och metod, 2006.

Robert Kaminski: Towards an XML Document Restructuring Framework, 2006.

Jiri Trnka: Prerequisites for data sharing in emergency management, 2007.

Björn Hägglund: A Framework for Designing Constraint Stores, 2007.

Daniel Andreasson: Slack-Time Aware Dynamic Routing Schemes for On-Chip Networks, 2007.

Magnus Ingmarsson: Modelling User Tasks and Intentions for Service Discovery in Ubiquitous Computing, 2007.

Gustaf Svedjemo: Ontology as Conceptual Schema when Modelling Historical Maps for Database Storage, 2007.

Gianpaolo Conte: Navigation Functionalities for an Autonomous UAV Helicopter, 2007.

Ola Leifler: User-Centric Critiquing in Command and Control: The DKExpert and ComPlan Approaches, 2007.

Henrik Svensson: Embodied simulation as off-line representation, 2007.

Zhiyuan He: System-on-Chip Test Scheduling with Defect-Probability and Temperature Considerations, 2007.

Jonas EImqvist: Components, Safety Interfaces and Compositional Analysis, 2007.

Håkan Sundblad: Question Classification in Question Answering Systems, 2007.

Magnus Lundqvist: Information Demand and Use: Improving Information Flow within Small-scale Business Contexts, 2007.

Martin Magnusson: Deductive Planning and Composite Actions in Temporal Action Logic, 2007.

Mikael Asplund: Restoring Consistency after Network Partitions, 2007.

Martin Fransson: Towards Individualized Drug Dosage - General Methods and Case Studies, 2007.

Karin Camara: A Visual Query Language Served by a Multi-sensor Environment, 2007.

David Broman: Safety, Security, and Semantic Aspects of Equation-Based Object-Oriented Languages and Environments, 2007.

Mikhail Chalabine: Invasive Interactive Parallelization, 2007.

Susanna Nilsson: A Holistic Approach to Usability Evaluations of Mixed Reality Systems, 2008.

Shanai Ardi: A Model and Implementation of a Security Plug-in for the Software Life Cycle, 2008.

Erik Kuiper: Mobility and Routing in a Delay-tolerant Network of Unmanned Aerial Vehicles, 2008.

Jana Rambusch: Situated Play, 2008.

Martin Karresand: Completing the Picture - Fragments and Back Again, 2008.

Per Nyblom: Dynamic Abstraction for Interleaved Task Planning and Execution, 2008.

Fredrik Lantz: Terrain Object Recognition and Context Fusion for Decision Support, 2008.

Martin Östlund: Assistance Plus: 3D-mediated Advice-giving on Pharmaceutical Products, 2008.

Håkan Lundvall: Automatic Parallelization using Pipelining for Equation-Based Simulation Languages, 2008.

Mirko Thorstensson: Using Observers for Model Based Data Collection in Distributed Tactical Operations, 2008.

Bahlol Rahimi: Implementation of Health Information Systems, 2008.

Maria Holmqvist: Word Alignment by Re-using Parallel Phrases, 2008.

Mattias Eriksson: Integrated Software Pipelining, 2009.

Annika Öhgren: Towards an Ontology Development Methodology for Small and Medium-sized Enterprises, 2009.

Rickard Holsmark: Deadlock Free Routing in Mesh Networks on Chip with Regions, 2009.

Sara Stymne: Compound Processing for Phrase-Based Statistical Machine Translation, 2009.

Tommy Ellqvist: Supporting Scientific Collaboration through Workflows and Provenance, 2009.

Fabian Segelström: Visualisations in Service Design, 2010.

Min Bao: System Level Techniques for Temperature-Aware Energy Optimization, 2010.

Mohammad Saifullah: Exploring Biologically Inspired Interactive Networks for Object Recognition, 2011 
Qiang Liu: Dealing with Missing Mappings and Structure in a Network of Ontologies, 2011.

Ruxandra Pop: Mapping Concurrent Applications to Multiprocessor Systems with Multithreaded Processors and Network on Chip-Based Interconnections, 2011.

Per-Magnus Olsson: Positioning Algorithms for Surveillance Using Unmanned Aerial Vehicles, 2011.

Anna Vapen: Contributions to Web Authentication for Untrusted Computers, 2011.

Loove Broms: Sustainable Interactions: Studies in the Design of Energy Awareness Artefacts, 2011.

Johan Blomkvist: Conceptualising Prototypes in Service Design, 2011.

Håkan Warnquist: Computer-Assisted Troubleshooting for Efficient Off-board Diagnosis, 2011.

Jakob Rosén: Predictable Real-Time Applications on Multiprocessor Systems-on-Chip, 2011.

Usman Dastgeer: Skeleton Programming for Heterogeneous GPU-based Systems, 2011.

David Landén: Complex Task Allocation for Delegation: From Theory to Practice, 2011.

Kristian Stavåker: Contributions to Parallel Simulation of Equation-Based Models on

Graphics Processing Units, 2011.

Mariusz Wzorek: Selected Aspects of Navigation and Path Planning in Unmanned Aircraft Systems, 2011.

Piotr Rudol: Increasing Autonomy of Unmanned Aircraft Systems Through the Use of Imaging Sensors, 2011.

Anders Carstensen: The Evolution of the Connector View Concept: Enterprise Models for Interoperability

Solutions in the Extended Enterprise, 2011.

Jody Foo: Computational Terminology: Exploring Bilingual and Monolingual Term Extraction, 2012.

Anders Fröberg: Models and Tools for Distributed User Interface Development, 2012.

Dimitar Nikolov: Optimizing Fault Tolerance for Real-Time Systems, 2012.

Dennis Andersson: Mission Experience: How to Model and Capture it to Enable Vicarious Learning, 2013.

Massimiliano Raciti: Anomaly Detection and its Adaptation: Studies on Cyber-physical Systems, 2013.

Banafsheh Khademhosseinieh: Towards an Approach for Efficiency Evaluation of

Enterprise Modeling Methods, 2013.

Amy Rankin: Resilience in High Risk Work: Analysing Adaptive Performance, 2013.

Martin Sjölund: Tools for Understanding, Debugging, and Simulation Performance Improvement of EquationBased Models, 2013.

Karl Hammar: Towards an Ontology Design Pattern Quality Model, 2013.

Maria Vasilevskaya: Designing Security-enhanced Embedded Systems: Bridging Two Islands of Expertise, 2013. Ekhiotz Vergara: Exploiting Energy Awareness in Mobile Communication, 2013.

Valentina Ivanova: Integration of Ontology Alignment and Ontology Debugging for Taxonomy Networks, 2014.

Dag Sonntag: A Study of Chain Graph Interpretations, 2014.

Kiril Kiryazov: Grounding Emotion Appraisal in Autonomous Humanoids, 2014.

Zlatan Dragisic: Completing the Is-a Structure in Description Logics Ontologies, 2014. 